\title{
Antenna Designs with Electromagnetic Band Gap Structures
}

\author{
Dalia M.N. Elsheakh, Hala A. Elsadek and Esmat A. Abdallah \\ Electronics Research Institute, Giza, \\ Egypt
}

\section{Introduction}

The word "meta", in Greek language, means beyond. It implies that the electromagnetic response of metamaterials (MTMs) is unachievable or unavailable in conventional materials. Many efforts have been done to search for an adequate definition for MTMs. In 2002, J.B. Pendry wrote in a conference paper: "meta-materials, materials whose permeability and permittivity derive from their structure". Later, in 2006, C. Caloz and T. Itoh wrote: "Electromagnetic metamaterials are broadly defined as artificial effectively homogeneous electromagnetic structures with unusual properties not readily available in nature" [1]. Perhaps, a serious obstacle on the road to a universal definition for the term MTMs is the fact that researchers working with these objects do not commonly agree on their most essential characteristics. In [2] and [3], some of the problematic aspects of the non-naturality definition were raised, like the difficulty in separating classical composites from the new class of metamaterials. Another argument against the "not found in nature" property is that it unnecessary excludes impressive examples of natural media that could be called metamaterials par excellence, such as structural colors [4].

MTMs cover an extremely large scientific domain which ranges from optics to nanoscience and from material science to antenna engineering. In this chapter, we focus primarily on the subject of MTMs in the electromagnetic field. Personally, We prefer the definition given by D.R. Smith: Electromagnetic metamaterials are artificially structured materials that are designed to interact with and control electromagnetic waves [5]. The term "artificial" refers to the fact that the electromagnetic response of these materials is dominated by scattering from periodically or amorphously placed inclusions (e.g., metallic or dielectric spheres, wires, and loops) [6].

In the family of MTMs, "left-handed" (LH) media drew an enormous amount of interest. This concept was first put forward by a Russian physicist, Victor Veselago, in 1968, for whom the medium is characterized by a simultaneously negative electric permittivity and negative magnetic permeability [7]. Veselago argued that such media are allowed by Maxwell's equations and that electromagnetic plane waves can propagate inside them, but the phase velocity of such a plane wave is in the opposite direction of the Poynting vector. Hence, some researchers use the term "backward wave media" (BWM) to describe these LH materials [8]. When such media are interfaced with conventional dielectrics, Snell's Law is reversed, leading to the negative refraction of an incident plane wave as shown in figure 1. 
Nevertheless, Veselago's conjecture was essentially ignored for thirty years due to the absence of naturally occurring materials or compounds that possess simultaneously negative permittivity and permeability.

In 2000, a metamaterial, based on conducting wires [9] and split-ring resonators (SRRs) [10], was demonstrated to have a negative refractive index over a certain range of microwave frequencies [10-13]. Wires, either continuous or with periodic breaks, can provide a positive or a negative effective permittivity. Planar SRRs or wound coils (also known as Swiss Rolls) can provide a positive or a negative effective permeability. Harnessing the phenomenon of negative refraction, these metamaterials offer a good potential for all kinds of applications, such as "perfect" lens [14], imaging [15], resonators [16], and cloaking [17].

Metamaterials possessing these properties are also frequently named "Negative Refractive Index (NRI)" and "Double Negative (DNG) material". In addition to the materials with simultaneously negative permittivity and negative permeability, the single negative metamaterials have also drawn a great interest. Applications are found for these materials either with a negative permittivity "Epsilon Negative (ENG)" [17] or a negative permeability "Mu Negative (MNG)" [18]. Besides, materials with the properties of "Epsilon near Zero (ENZ)" [19] and "Mu Near Zero (MNZ)", known as "nihility" materials have also been studied. A simple synopsis of these metamaterials can be found in figure 2, where the angular frequencies $\omega p e$ and $\omega p m$ represent, respectively the electric and magnetic plasma frequency [20]. Up to now, we talked about metamaterials who exhibit their great performances by artificially tailoring the permittivity or permeability. Besides, the term "metamaterial" has also been used by some authors to describe other periodic structures such as electromagnetic bandgap (EBG) structures or photonic crystals, when the period is much smaller in physical size than the wavelength of the impinging electromagnetic wave. The electromagnetic response of such structures is dominated by Bragg-type scattering and involves higher order spatial harmonics (Bloch-Floquet modes) [20]. In this chapter, we focus on such a kind of metamaterial, the so-called "electromagnetic band gap" (EBG). Electromagnetic has received great attention among researchers all over the world because of its immense civilian and defense applications. During the Second World War, the use of radar and thereafter the wide use of microwave communication systems facilitated the transformation from radio to microwave frequency. This dramatic change demanded more advanced materials for high frequency performance and opened up new dimensions in the field of electromagnetic materials. Nano-composites and electromagnetic band-gap structures are examples of metamaterials under right hand rules. Electromagnetic band-gap (EBG) structures have attracted increasing interest in the electromagnetic community. Because of their desirable electromagnetic properties [21], they have been widely studied for potential applications in antenna engineering. Hundreds of EBG papers have been published in various journals and conferences in the last 5 years. EBG are periodic arrangements of dielectric or metallic elements in one, two or three dimensional manners. EBG inhibits the passage of electromagnetic wave at certain angles of incidence at some frequencies. These frequencies are called partial band-gap. At a specific frequency band, EBG does not allow the propagation of wave in all directions and this frequency region is called the complete band-gap or global band-gap [22, 23]. Physicists put the original idea of EBG forward and some recent studies revealed the interesting fact that EBG exists in living organisms. The well known examples are the butterfly wing scales and eyes of some insects. In this case, a metallic like reflection effect is obtained by using refractive index differences. 
A multilayer thin film with different refractive indices in animals is a good example for this. Recently, these ideas were undergone a preliminary study for its commercialization such as paints for certain applications.

The concept of electromagnetic band-gap (EBG) structures originates from the solid-state physics and optic domain, where photonic crystals with forbidden band-gap for light emissions were proposed in [27-28] and then widely investigated in the [29-33]. Thus, the terminology, photonic band-gap (PBG) structures, was popularly used in the early days. Since then, a profusion of scientific creativity has been witnessed as new forms of electromagnetic structures are invented for radio frequency and microwaves. EBG can be realized in one, two and three dimensional forms. The dimensionality depends on the periodicity directions. Three dimensional EBG are more appropriate for getting a complete band-gap because they can inhibit waves for all incident angles. The band-gap in EBG is analogous to a forbidden energy gap in electronic crystals. Hence EBG are also termed as photonic crystals (PCs). The first attempts towards three-dimensional structures were realized in the form of face centered cubic (fcc) lattice structures [35]. At the initial stages of EBG research, due to the lack of theoretical predictions, a 'cut and try' approach was adopted in experimentally predicting the band-gap. At the beginning, the investigations of EBG were mainly on wave interactions of these structures at optical frequencies and hence PBG emerged with the name of photonic band-gap structures. Now, vast extensions of EBG at microwave [36], millimetre [37] and sub-millimetre wave frequencies [38] are electromagnetic band-gap (EBG) structures. A periodic structure can give rise to multiple band-gaps. However, it should be noted that the band-gap in EBG is not only due to the periodicity of the structure but also due to the individual resonance of one element. A study revealed the mechanisms to form a band-gap in an EBG [21]. The band-gap formation in EBG is due to the interplay between macroscopic and microscopic resonances of a periodic structure. The periodicity governs the macroscopic resonance or the Bragg resonance. It is also called the lattice resonance. Microscopic resonance is due to the element characteristics and it is called the Mie resonance [20]. When the two resonances coincide, the structure possesses a band-gap having maximum width. Depending on the structural characteristics and polarization of the wave, one resonance mechanism (i.e. either the multiple scattering resonance or the single element scattered resonances) can dominate over the other. The characteristic property of stop bands at certain frequencies enables many applications using EBG. At this stop band, all electromagnetic wave will be reflected back and the structure will act like a mirror. At other frequencies, it will act as transparent medium. This concept is illustrated in figure 3.

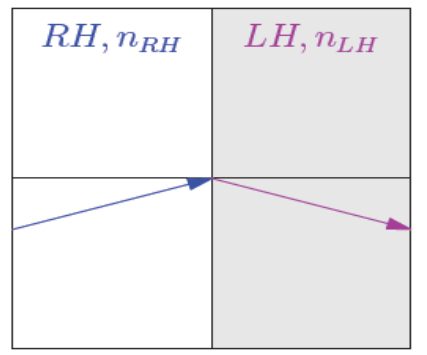

Fig. 1. A negative reflection. 


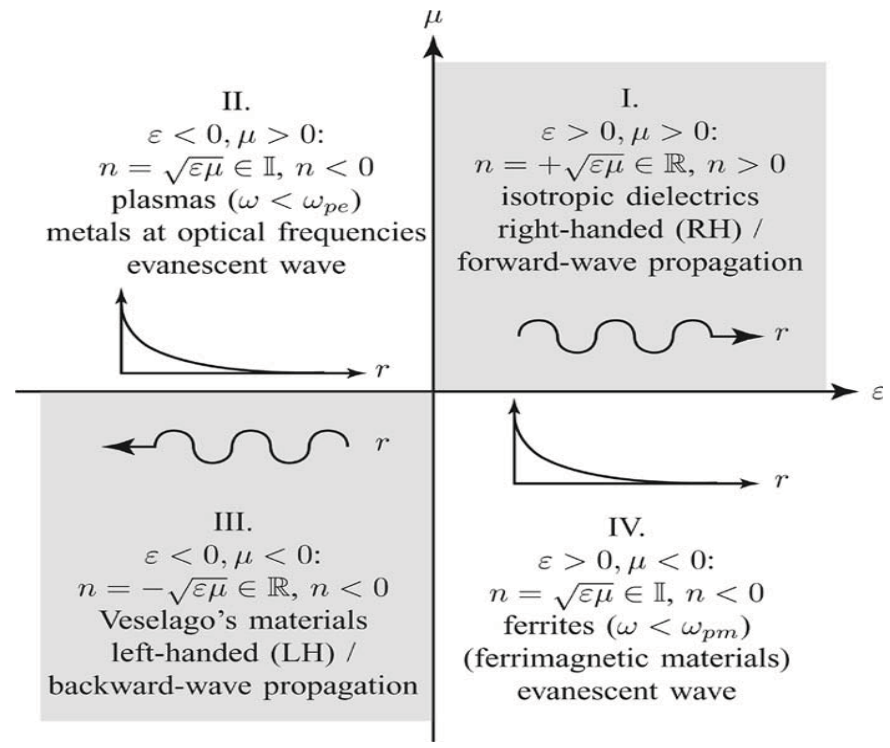

Fig. 2. Permittivity, permeability and refractive index diagram [20].
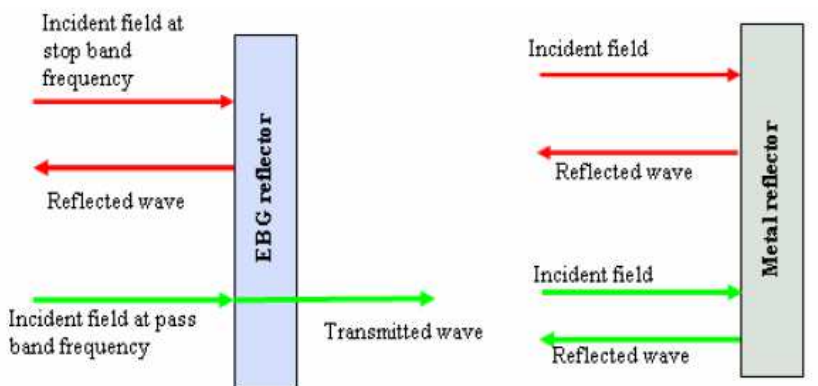

Fig. 3. Diagram illustrating the application of EBG as a mirror and its comparison with a metal reflector [21].

Microstrip antennas mounted on a substrate can radiate only a small amount of its power into free space because of the power leak through the dielectric substrate [32]. In order to increase the efficiency of the antenna, the propagation through the substrate must be prohibited. In this case, the antenna can radiate more towards the main beam direction and hence increase its efficiency. Recently, there has been much interest in the field of Metallo-Dielectric Electromagnetic Band-Gap (MDEBG) structures because of the promising future applications and the important role these artificially engineered periodic materials may play in the field of antennas. The name "Photonic (or Electromagnetic, which is more appropriate for the frequency band of applications) band-gap" has its origin in the fact these structures effectively prevent the propagation of electromagnetic waves within a specific frequency range (the band gap). Two examples of the qualitative geometry of such structures are given in figure 4 [33]. As shown in figure 4, a MDEBG structure is essentially a surface comprising a plurality of elements. Each of the elements is interconnected with each other to form an array of metallic 
parts embedded in a slab of dielectric. In other words, they are periodical structures of densely packed planar conducting patches separated from a solid metal plane by a dielectric layer. Sometimes metallic pins (or via) are introduced to prevent electromagnetic waves from traveling in the waveguide between the array and the ground. Each unit cell, which is periodically repeated to form the array, essentially behaves as a microwave resonant circuit. The plurality of the resonant elements is parameterized to substantially block surface wave's propagation in the device within a predetermined frequency band gap [35].

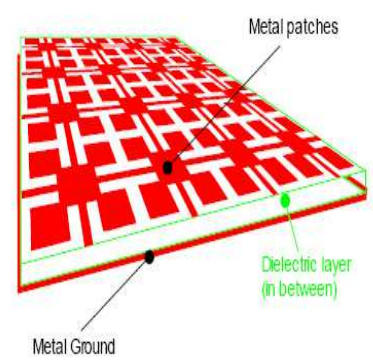

(a)

Dielectric slab

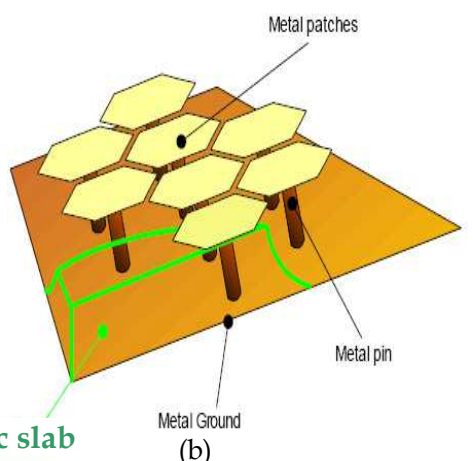

(b)

Fig. 4. Simple examples of Metallo-Dielectric EBG structures [32].

The objective of this chapter is to investigate the EM properties of the microstrip antennas based on metallo-dielectric electromagnetic band-gap structures. It is important to emphasize the fact that simple embedded electromagnetic band-gap (EEBG) structures presented in this work are targeting operating frequency band at $0.5-20 \mathrm{GHz}$ range. Since simple EEBG structures have impractical geometrical sizes in the $500 \mathrm{MHz}$ to $2 \mathrm{GHz}$ frequency range, more complex EEBG structures need to be employed. In addition, from wireless communication application, miniaturization of electronic systems requires the availability of miniaturized EEBG structures with appropriate patch sizes. The concept introduced in this chapter can be generally applied regardless of the size of the EEBG structures. For wideband radiation, reduction from hundreds of $\mathrm{MHz}$ to few $\mathrm{GHz}$ either a combination of different methods or use of advanced EEBG structures is the best solution as shown in figure 5 [40]. Figure 5 shows the efficacy range of EEBG structures covered in [40].

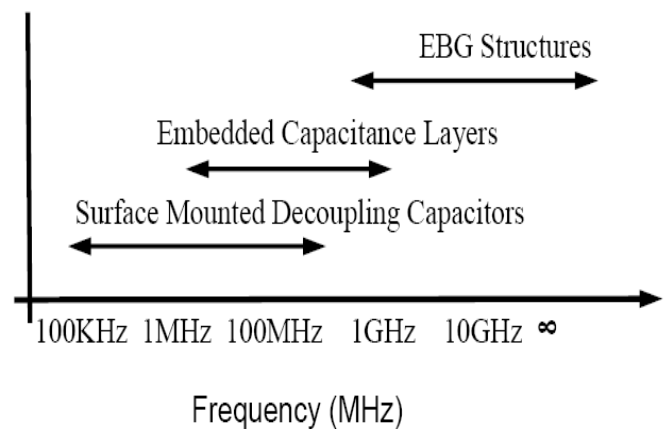

Fig. 5. Efficacy range of different reduction methods. 


\section{Disadvantages of metal ground planes and the MDEBG solution}

MDEBG structures are useful where the presence of classic electric conductors as antenna ground planes adversely affects the performance of the entire electromagnetic device. As it is known, classic conductive surfaces are extensively used as antenna reflectors: they redirect one half of the radiation into the opposite direction potentially improving the antenna gain by $3 \mathrm{dBi}$.
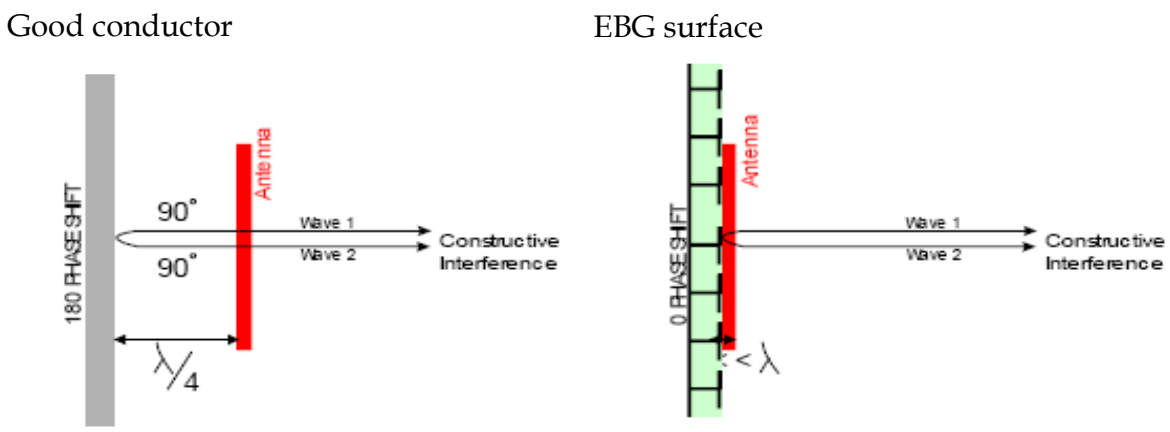

Fig. 6 . An antenna separated by $1 / 4 \lambda$ from the ground plane (on the left) and the alternative MDEBG layout (on the right).

However, they do have two main disadvantages: first, they reverse the phase of the reflected wave and second, they support propagating surface waves, which can have unwanted effects on the antenna performance. The fact that they reverse the phase 180 degrees is due to the most obvious constraint that the tangent electric field on a classic conductive surface must be zero, so the electromagnetic waves experience a 180 degrees phase shift on reflection. Because of the phase reversal, the image currents cancel the antenna currents, resulting in poor radiation efficiency when the antenna is too close to the conductive surface. This problem is often solved by including a quarter wavelength between the radiating element and the ground plane (see figure 6), but the disadvantage of this solution is the fact that the structure requires a minimum thickness of $\lambda / 4$. It will be shown that, by using the novel MDEBG structures as ground planes, the antenna can be almost be attached to the ground plane, resulting in a useful reduction of volume. It will be proved that, at the frequency where the MDEBG structure does not give any reflection phase shift, a design of MDEBG structures even 8 times thinner than the classic ones (which implement a $\lambda / 4$ spacing between antenna and ground plane) is possible. As stated before, another issue is the propagation of surface waves when normal ground planes are used: these are propagating electromagnetic waves bound to the interface between metal and free space and they will radiate if scattered by bends, discontinuities or surface textures. The unwanted result is a kind of multipath interference, which can be seen as ripples in the radiation pattern (see figure 7) [41].

Again, by using MDEBG structures, it will be shown that surface waves can be suppressed. It follows that, when multiple antennas share the same normal conductive ground plane, like it happens in phased arrays, the above mentioned surface waves may cause undesired mutual coupling between the antennas (see figure 8). Once again, by using MDEBG surfaces structures, it is possible to alter the surfaces properties of the ground plane and avoid this 
mutual coupling. Therefore, it can be easily understood that the Metallo-Dielectric EBG structure is a useful alternative to antenna classic metallic reflectors.

From above discussion, to realized high performance antenna:

- A reflector which lacks edge currents that radiate power into the back hemisphere of the antenna is needed;

- Surface waves on a ground plane associated with an antenna have to be suppressed to provide more efficient antennas, and reduce coupling.
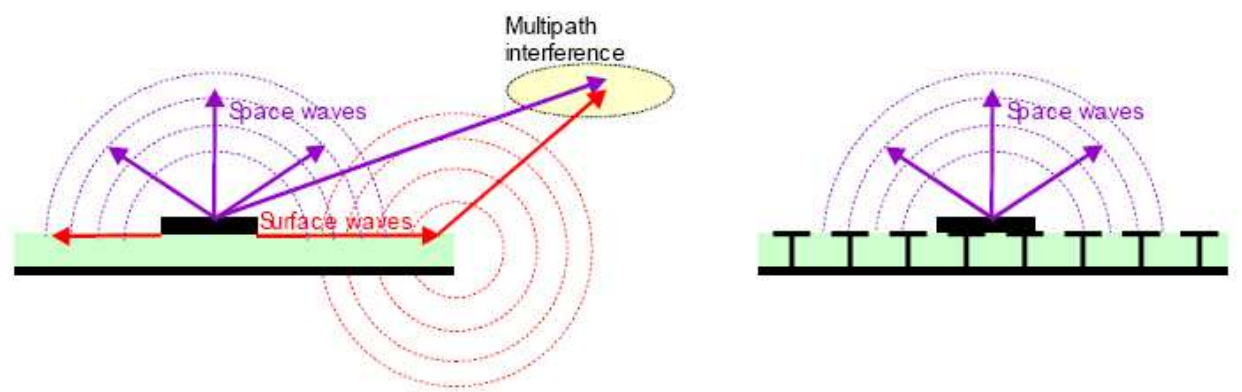

Fig. 7. Multipath interference due to surface waves on a normal ground plane (on the left) and the alternative MDEBG layout (on the right).

Nowadays, one of the most commonly used solution to prevent the propagation of unwanted surface waves is the so called "choke ring" which provides excellent electrical performance for GPS antennas. They are usually very large and heavy. While size and weight are not issues for most base station applications, for a GPS surveyor carrying one around in the field, size and weight are important factors. Moreover, choke rings are also expensive, typically costing thousands of dollars.

\section{Metallo EBG structures as novel ground planes for antenna applications}

The modern trends in communication systems require wide bandwidth, small size and low profile antennas. A planar microstrip antenna (PMA) is the good candidate for use in UWB wireless technology because they can reach wide impedance bandwidth and nearly omnidirectional azimuthally radiation pattern by different methods. One simple but powerful technique is to replace the cylindrical wires with the plate elements, such as rectangular (square), elliptical (circular), triangular shapes, and others. Another way to increase the impedance bandwidth of the monopole antennas can be achieved by modifying the ground plane. Different shapes of modified ground plane as semi circular is used to increase bandwidth. With the intention to overcome this handicap, a thick, high permittivity substrate is used, and potential surface waves are suppressed applying so called Electromagnetic Band-gap (EBG) [43]. The surface wave propagation is a serious problem of MPA. Surface waves reduce antenna efficiency and gain, limit bandwidth, increase end-fire radiation and cross-polarization levels. To avoid this, the substrate is periodically loaded so that the surface waves cannot propagate along the substrate. Also, other surface wave coupling effects like mutual coupling between array elements and interference with board systems can be suppressed [44]. 


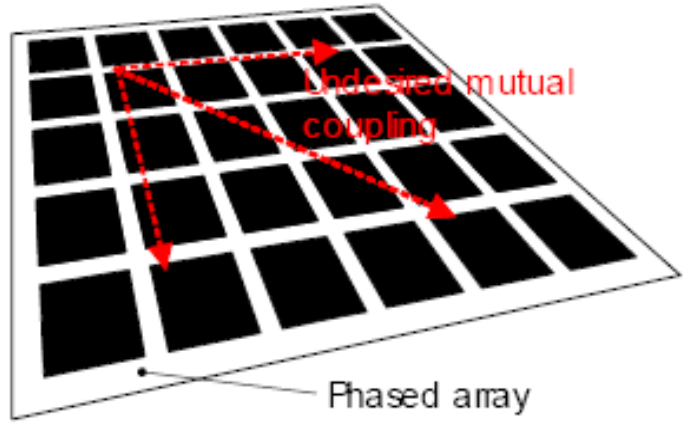

Fig. 8. Mutual coupling for multiple antennas due to the presence of surface waves.

To avoid using costly hybrid technology, innovative design must be developed to successfully integrate antenna with circuitry on high dielectric substrates. The novel RF system architecture reduces size, weight, losses and it is also suitable for integration with micro-electromechanical systems to realize reconfigurable circuits and antennas. Two technologies have been mainly pursued so far to achieve MPA on high-dielectric substrate with optimum performance. One is based on micromachining technology hile the other makes use of the concept of photonic band-gap substrates

A defected ground structure (DGS) has gained significant interests. It rejects certain frequency bands, and hence it is called electromagnetic band-gap (EBG) structures. Liu et al. has presented a novel DGS based meander microstrip line providing a broad stop-band [46]. A novel defected ground structure with islands (DGSI) is proposed by T. Itoh. in [48]. The DGS is realized on the bottom plane with two islands placed at both sides of the microstrip line on the upper plane. Due to their excellent pass and rejection frequency band characteristics, DGS circuits are widely used in various active and passive microwave and millimeter-wave devices such as filters, dividers, couplers, amplifiers, resonators and antennas.

\section{Applications}

One of the main purposes of this chapter is to deeply go into all the promising possible applications of MDEBG structures by satisfy their challenging design requirements of the antennas. Medical application as image scan is very important nowadays. A very promising way to eradicate the problems created by surface waves in this application (e.g. scan blindness), while at the same time improving performance, is to use electromagnetic bandgap structures instead of standard dielectric antenna substrates. Within the MDEBG, in fact, the unwanted effect of surface waves will be efficiently suppressed.

Another microwave application is high precision GPS. High precision GPS is going to be used in many situations. By very accurately determining the phase of the signal, it is hoped to reach a position accuracy of a few millimeters. However, in order to avoid errors in measurements (due to multi-path), the backward radiated field has to be at least a few orders of magnitude below the frontward field. Such a requirement cannot be obtained if the antenna excites surface waves and electromagnetic band-gap materials could again be used as design solutions. Another application is mobile and wireless communications. As 
the world goes, wireless, data and voice transmissions are bound to become even more common. A lot of attention is now focused on Bluetooth band hence its implementation in different wireless systems increases in everyday life. Moreover, for other applications like cell-phones, more attention is being paid to the shielding offered by the antenna and the potential health hazard.

Other than that, the EBG structure also can be used as a band reject especially for ultra wide band applications which operate at very wide frequency ranges. The simulation and measurement results for ultra wide band with and without band rejection are investigated in this chapter.

EBG structures are used to prevent some operating modes and make harmonic control. These techniques can increase the usability of antenna systems. The design and simulation of the antennas with EBG structures have recently received more attention. Initial concepts only have been proven with limited fabricated devices. A lot of research efforts is still expected in this field. This chapter is a step to investigate and develop how the EBG structure/ground plane can be used to optimize the antenna performance to satisfy several applications. The investigated antennas are miniaturized with broadband characteristics to be suitable for multi-band/ multi-function operation in wireless communications and medical applications.

\section{Problems led to the use of EBG}

Antenna designs have experienced enormous advances in the past several decades and they are still under research and development. Many new technologies have emerged in the modern antenna design arena and one exciting breakthrough is the discovery/ development of EBG structures. The applications of EBG structures in antenna designs have become interesting topic for antenna scientists and engineers. The recent explosion in antenna developments has been fueled by the increasing popularity of wireless communication systems and devices. From the traditional radio and TV broadcast systems to the advanced satellite system and wireless local area networks, wireless communications have evolved into an indispensable part of people's daily lives. Antennas play a paramount role in the development of modern wireless communication devices, ranging from cell phones to portable GPS navigators, and from the network cards of laptops to the receivers of satellite TV. A series of design requirements, such as low profile, compact size, broad bandwidth, and multiple functionalities, keep on challenging antenna researchers and propelling the development of new antennas. Progress in computational electromagnetic, as another important driving force, has substantially contributed to the rapid development of novel antenna designs. It has greatly expanded the antenna researchers' capabilities in improving and optimizing their designs efficiently.

Various numerical techniques, such as the method of moments (MOM), finite element method (FEM), and the finite difference time domain (FDTD) method, have been well developed over the years. As a consequence, numerous commercial software packages have emerged. Nowadays with powerful personal computers and advanced numerical techniques or commercial software, antenna researchers are able to exploit complex electromagnetic materials in antenna designs, resulting in many novel and efficient antenna structures with most of required characteristics. 
For these reasons, EBG structures and their applications in antennas have become a new research direction in the antenna researches community. It was first proposed to respond to some antenna challenges in wireless communications [40]. For example,

- How to suppress surface waves in the antenna ground plane?

- How to design an efficient low profile antenna near a ground plane?

- How to increase the gain of an antenna?

In the novel RF system architecture of reducing the size, weight, losses and suitability for integration with micro-electro-machine-system lead to achieve reconfigurable antenna with required features. If a conventional substrate is used, then most of the antenna radiation is emitted from the substrate (since it has a higher dielectric constant than air). A big part of this radiation is trapped inside the substrate because of total internal reflection. As a consequence of this, more than $50 \%$ of the radiated energy is lost. Also heat dissipation and temperature effects arise in the substrate.

Two technologies have been used to achieve microstrip antenna on high dielectric substrate with optimum performance:

- $\quad$ One is based on micromachining technology.

- $\quad$ Second is photonic band-gap (PBG) substrate.

A photonic crystal essentially behaves much like a band-stop filter, rejecting the propagation of energy over a fixed band of frequencies. An appropriate EBG substrate is selected, then all of the energy can be directed towards the radiating direction (total reflection by the EBG structure), thus improving the antenna directivity and eliminating the substrate heat dissipation [41].

Due to the complexity of the EBG structures, it is usually difficult to characterize them through analytical methods. Instead, full wave simulators that are based on advanced numerical methods have been popularly used in EBG analysis. Dispersion diagram, surface impedance, and reflection phase features are explored for different famous EBG structures. The interaction of antennas and EBG structures are extensively investigated. In summary, the EBG research has flourished since the beginning of this new millennium.

\section{Electromagnetic Band-Gap (EBG) structure}

\subsection{The parameters of EBG (figure 9)}

- Permittivity of the dielectric materials used $\left(\varepsilon_{\mathrm{r}}\right)$

- Dimensions of the mushroom patches (a)

- $\quad$ Periodicity $(\mathrm{P})$

- Incident angle of electromagnetic waves $\left(\theta_{\mathrm{i}}\right)$

A periodic structure is characterized by the following parameters:

1. $\lambda_{\mathrm{r}}=2 . \mathrm{a}$

2. Shape of individual patches.

3. Filling factor ratio between size of the patches and the periodicity of unit cell (a/P).

For best performance [40].

- $\quad$ For 3D-EBG $0.9<\mathrm{a} / \mathrm{P}<0.95$

- $\quad$ For 2D-EBG $0.65<\mathrm{a} / \mathrm{P}<0.75$ 


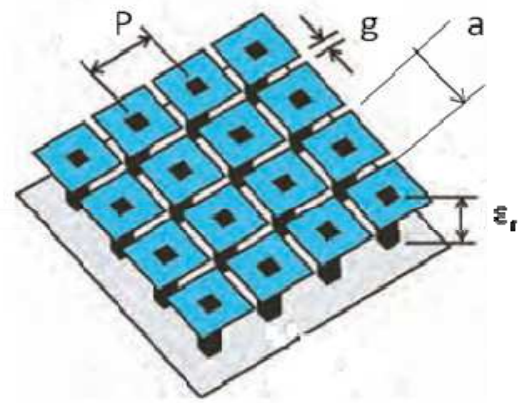

Fig. 9. The parameters of EBG.

\subsection{The features of EBG}

The main feature of EBG structures is their capability to affect the radiative dynamics within the structure so that there are no electromagnetic modes available within the dielectric. This feature is analogous to periodically arranged atomic lattice of a semiconductor which gives rise to the allowed values of energy that an electron can have at the valence band and at the conduction band, with an energy band-gap separating the two. The optical analogy to this situation is a periodic dielectric structure with alternating high and low values of permittivity, which gives rise to a photonic band-gap [50].

\subsection{Applications of EBG}

In optical domain new highly efficient opto-electronic devices are considered such as very efficient laser diodes [44, 45], microscale light circuits, multiplexers or demultiplexers based on inhibition of spontaneous emission, photoluminescence, wave-guiding, and superprism phenomenon [46-49]. Studies of frequencies occurring for metal photonic crystals have also shown that the frequency can be controlled and could appear in the microwave region [50]. In the microwave domain, many developments concern the direct control of the electromagnetic energy and its transmission: mirrors, electromagnetic windows, and radiation pattern control. We find also the high impedance material of Sievenpiper et al [51]. They proposed their structure as perfect magnetic wall to reduce the leaky waves in antenna array. The material developed allows the realization of antennas, low loss coplanar lines and compact integrated filter [52-54].

Other applications include duplexers and controllable PBG materials. Due to a certain easiness of fabrication in this frequency domain, the challenge of the electronically controlled photonic crystals has a significant interest. Industrial applications of these crystals are under development, concern mainly aerospace, and telecom domains [43].

\subsubsection{Antenna substrates for surface wave suppressions}

Surface waves are by-products in many antenna designs. It directs electromagnetic wave propagation along the ground plane instead of radiation into free space, consequently reduce the antenna efficiency and gain. The diffraction of surface waves increases the back lobe radiations, which may deteriorate the signal to noise ratio in wireless communication 
systems such as GPS receivers. In addition, surface waves raise the mutual coupling levels in array designs as shown in figure 10, resulting in the blind scanning angles in phased array systems. The band-gap feature of EBG structures has found useful applications in suppressing the surface waves in various antenna designs. For example, an EBG structure is used to surround a microstrip antenna to increase the antenna gain and reduce the back lobe as shown in figure 7. In addition, it is used to replace the quarter-wavelength choke rings in GPS antenna designs. Many array antennas also integrate EBG structures to reduce the mutual coupling level more over to increase antenna gain used super-state EBG as shown in figure 10 [40].

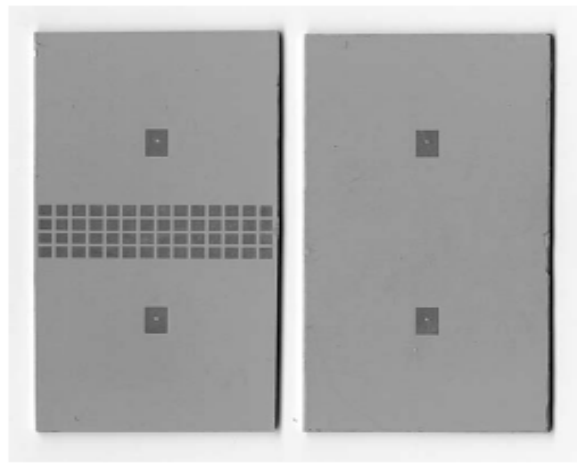

Fig. 10. EBG substrate for surface wave suppression with low mutual coupling microstrip array.

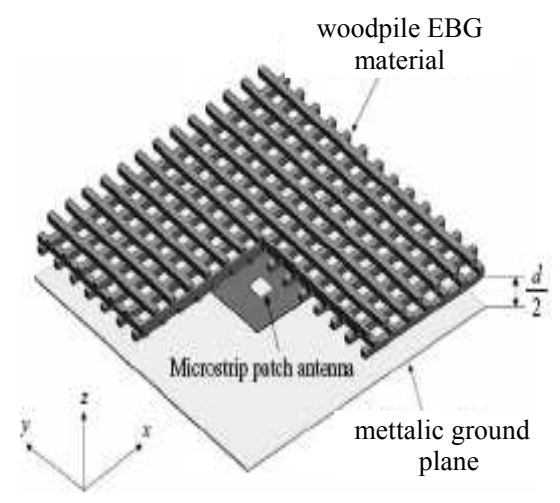

Fig. 11. A high gain resonator antenna design using a woodpile EBG structure.

\subsubsection{Antenna substrates for efficient low profile antenna design}

Another favorable application of EBG is to design low profile wire antennas with good radiation efficiency, as shown in figure 11, which is desired in modern wireless communication systems. To illustrate the fundamental principle, Table 1 compares the EBG with the traditional PEC ground plane in antenna designs [43]. 


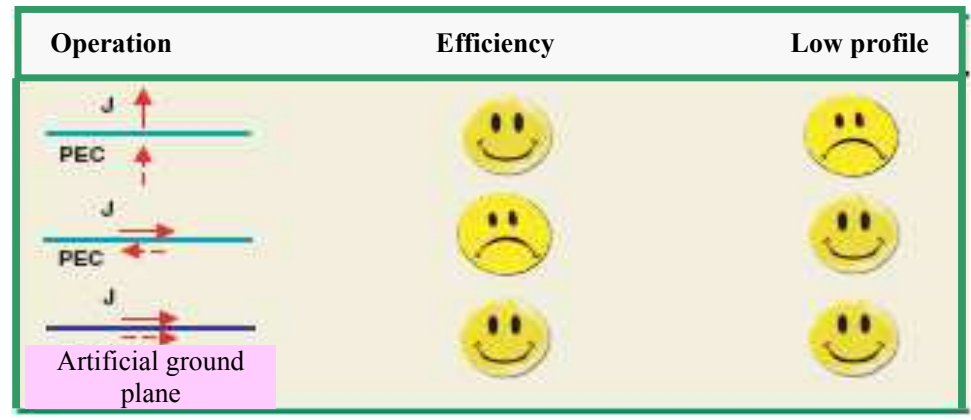

Table 1. Comparison of conventional PEC and artificial ground planes in antenna designs [43].

When an electric current is vertical to a PEC ground plane, the image current has the same direction and reinforces the radiation from the original current. Thus, this antenna has good radiation efficiency, but suffers from relative large antenna height due to the vertical placement of the current. To realize a low profile configuration, one may position an antenna horizontally close to the ground plane. However, the problem is the poor radiation efficiency because the opposite image current cancels the radiation from the original current. In contrast, the EBG surface is capable of providing a constructive image current within a certain frequency band, resulting in good radiation efficiency. In summary, the EBG surface exhibits a great potential for low profile efficient antenna applications as shown in figure 12. Based on this concept, various antennas have been constructed on the EBG ground plane [59-62]. Typical configurations include dipole antenna, monopole antenna, and spiral antenna. EBG surfaces have also been optimized to realize better performance such as multiband and wideband designs.

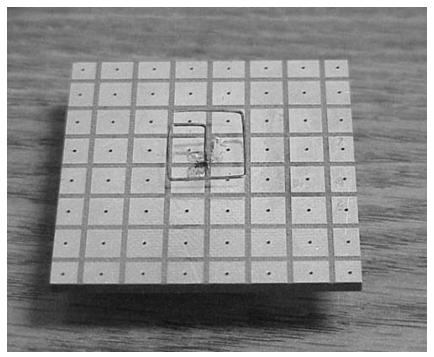

Fig. 12. EBG substrate with low profile antenna.

\subsection{Advantages of EBG}

Utilizing electromagnetic band-gap crystals in a patch antenna with an air gap appears to perform five key functions:

4. Increase operation bandwidth

5. Reduce side-lobe levels

6. Increase front to back $(\mathrm{F} / \mathrm{B})$ ratios

7. Increase directivity and consequently gain improvement

8. Harmonic control (suppression of resonance at the harmonic frequencies of the antenna) 


\section{Classifications of electromagnetic band-gap structures}

\subsection{Defected Ground Structures (DGS)}

A Defected Ground Structure (DGS) is an etched lattice shape, which is located on the ground plane. DGS has arbitrary shapes and is located on the backside metallic ground plane. DGS is realized on the bottom plane with one island placed at both sides of the microstrip line on the upper plane. DGS for the microtrip line, which has etched defects in the backside metallic ground plane, is one hotspot concepts of microwave circuit design nowadays. Compared to photonic band-gap (PBG), DGS has simple structure and potentially great applicability to design microwave circuits such as filters, amplifiers and oscillators. DGSs have gained significant interests. It rejects certain frequency bands, and hence it is called electromagnetic band-gap (EBG) structures as shown in figure 13 [57]. The DGS cell has a simple geometrical shape, such as rectangle. Novel fractal DGS cell is proposed. Its band-gap and slow-wave characteristics are better than the conventional ground plane. DGSs have gained quite significance in filter design [58] showing optimal pass-band and stop-band responses plus sharp selectivity and ripple rejection. Application of CPW-based spiral-shaped DGS to MMIC for reduced phase noise oscillator [57], active devices (BJT and FET) can also be mounted using DGS technique. High amount of isolation is achieved in microstrip diplexer and harmonic control can also be achieved on microstrip antenna structures using DGS. Figure 14 gives the schematic of such a DGS with its approximate surface area. A novel DGS based meander microstrip line providing a broad stop band is presented in [59]. Novel Defected Ground Structures with Islands (DGSI) is proposed in [58]. Careful selection of the line width guarantees $50 \Omega$ characteristic impedance $\left(Z_{0}\right)$.

The EM simulation results of the DGSI are compared with circuit simulation results using extracted parameters; showing excellent agreement between the two in wide band. Examination of stop band characteristics is studied using concentric circular rings in different configurations. Metallic backing significantly reduces interference effects, harmonics and phase noise. Several novel 1D DGS are presented for microwave integrated circuits (MIC), monolithic MIC (MMIC), low temperature cored ceramic (LTCC) including RF front-end applications. Significant change in the characteristics including slow-wave factor (SWF) of periodic structures like transmission lines is achieved using quite a few unconventional DGS like spiral-shaped and vertically periodic DGS. Vertically periodic DGSs (VPDGS) have been used in reducing the size of MIC and amplifiers, thus increasing SWF significantly. Harmonic control can also be achieved on microstrip antenna structures using 1-D DGS [59].

Table 2 presents the difference between defected ground plane structure and band-gap structure. The characteristics of the defected ground structure are:

- Disturbs shielding fields on the ground plane.

- Increases effective permittivity.

- Increases effective capacitance and inductance of transmission line.

- Has one-pole LPF characteristics (3dB cutoff and resonance frequency).

- $\quad$ Size reduction for the component. 


\begin{tabular}{|c|c|c|}
\hline Comparisons & $\begin{array}{c}\text { Electromagnetic Band-gap } \\
\text { Structure (EBG) }\end{array}$ & $\begin{array}{c}\text { Defected Ground Structure } \\
\text { DGS }\end{array}$ \\
\hline Geometry & Periodic etched structure & One or few etched structure \\
\hline $\begin{array}{c}\text { Microwave } \\
\text { Circuit properties }\end{array}$ & Similar & Similar \\
\hline $\begin{array}{c}\text { Equivalent } \\
\text { Circuit extraction }\end{array}$ & Very difficult & Relatively simple \\
\hline
\end{tabular}

Table 2. Comparison between defected ground structure and EBG..

\subsection{Photonic Band-Gap (PBG) structures}

\subsubsection{Historical background of PBG}

Photonic band-gap (PBG) structures are periodic structures that manipulate electromagnetic radiation in a manner similar to semiconductor devices manipulating electrons. Semiconductor material exhibits an electronic band-gap where there are electrons cannot exist. Similarly, a photonic crystal that contains a photonic band-gap does not allow the propagation of electromagnetic radiation with specific frequencies within the band-gap [60]. This phenomenon results from the destructive Bragg diffraction interference due to the periodic boundary conditions of PBG structures. This property has a significant importance in many microwave and optical applications to improve their efficiency. The photonic bandgap structures were first investigated in [61] by Yablonovitch. He introduced vectorspherical-wave expansion method or the vector Koringa-Kohn-Rostker (KKR) method to calculate the dispersion relation and the transmittance for the regular array of dielectric spheres. This was the first self-consistent treatment of the electromagnetic eigen modes in 3$\mathrm{D}$ dielectric systems with large periodic modulation of the dielectric constant. A remarkable step was made by Yablonovitch who pointed out the possibility of the realization of photonic band-gap, localized defect modes, and their applications to various optoelectronic devices. His ideas stimulated many researchers, and energetic research activities including his own studies were initiated. The process to realize the photonic band-gap is described in [62]. Lee et al [49] discussed the strong localization of electromagnetic waves in disordered PBG structures.

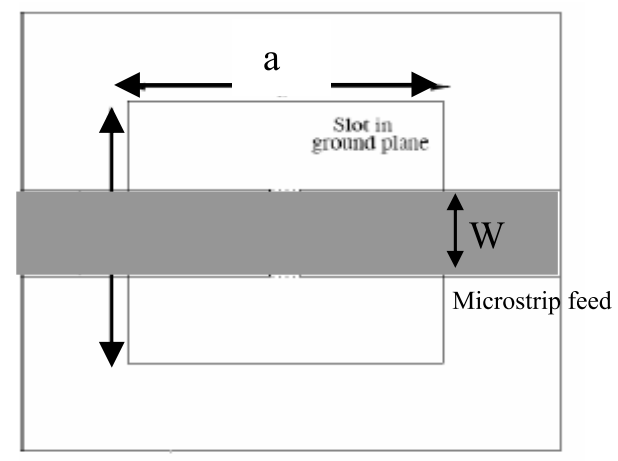

Fig. 13. Schematic diagram of a unit DGS cell. 

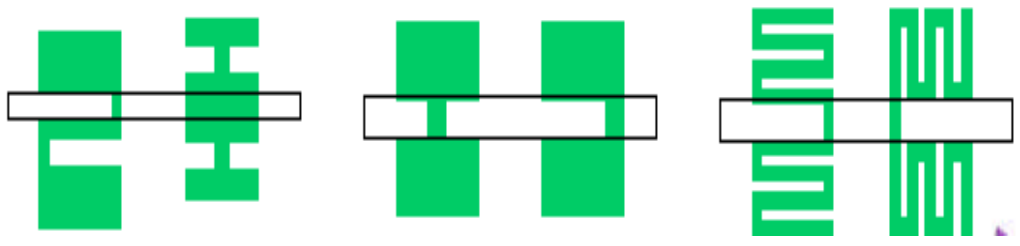

Fig. 14. Different shapes of DGS structures.

Since then, many researchers in various fields such as physics, electronics, waves, optics, fabrication, and chemistry have been engaged in the realization of photonic band-gap, localized defect modes, and other microwave and optical properties peculiar to the PBG structures [63]. They have also been collaborating to make new structures and measure their properties. PBG structures were initially applied to optical applications like high-quality optical mirrors and resonators. One of the most exciting projected applications of PBG structures is the production of optical circuits by manipulating light with optical waveguides. Currently, fiber-optic signals must be converted to electronic signals in order to be analyzed by computers or other devices connected to the optical line. This slows the signal considerably and the electronic circuitry is fairly inefficient as well. A device that runs completely on light would revolutionize technology in several areas, leading eventually to an all optical network (AON).

The integration of photonic components such as lasers, detectors, couplers, and waveguides is still at a very primitive stage due to difficulties with implementing integrated optical components smaller than certain sizes. For instance, small bends or curves of waveguides lead to the leakages of optical signals, so the bends have to be bigger than certain length. PBG structures are proposed as a possible solution to this problem. Adding a defect in a PBG structure opens a path along which electro-magnetic radiation can propagate. Because the PBG structure can be tailored to completely reflect certain frequencies, it is possible to turn corners with light at a distance of the light's wavelength order. Another application of PBG structures is the use of these crystals to greatly improve the efficiency of lasers and light-emitting diodes. Due to the scalability of PBG structures, several applications at microwave and millimeter-wave frequencies have been developed [64]. Other applications such as resonant cavities can be designed by using PBG structures are also presented. Applications based on the guiding and localized mode properties of the PBG structures are given. Some of these common applications in both microwaves and optics are power splitters, switches, directional couplers, high quality filters, and channel drop filters.

\subsubsection{Basic PBG structures}

Figure 15, 16 shows different configurations of PBG structures composed of two different materials. These configurations include 1D, 2D and 3D periodicities. The two different materials of a PBG structure can be two different dielectric materials or a metal and a dielectric material. Metallic photonic band-gap systems have received far less attention than dielectric PBG structures. However, it has been suggested that periodic metallic structures have important applications, such as cavities, waveguides, and antennas [65]. The main advantage of dielectric PBG structures is that they do not include metallic loss which is 
usually the dominant loss at high frequencies. Dielectric materials that can be used to construct PBG structures are widely available for almost all frequency bands from ultraviolet to microwave range which facilitate the design and fabrication. Many basic characteristics are common in both ordinary and photonic crystals. These characteristics are utilized to build the fundamental theories of photonic crystals. However, a major difference between ordinary and photonic crystals is the scale of the lattice constant. In the case of ordinary crystals, the lattice constant is in the order of angstroms. On the other hand, lattice constant is constructed with millimeter dimensions for microwave range. Figure 16 shows examples of different PBG structures used in the design of different applications. Various technologies have been developed and applied for manufacturing PBG structures in the last ten years such as stacking slabs of 2D materials for 3D structures implementations. For practical implementation of an infinite 2D PBG structure, a 2D PBG slab of finite thickness is surrounded by two perfect electric conductor (PEC) plates. This modification is implemented to guide the waves in the normal direction to the periodic cell. In optical applications, confinement of electromagnetic waves inside a 2D PBG slab can be obtained by surrounding the slab by a different material of another dielectric constant [66].

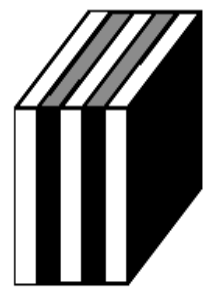

(a)

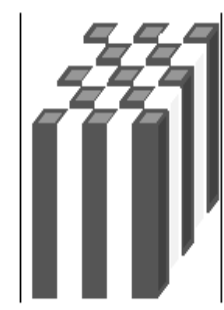

(b)

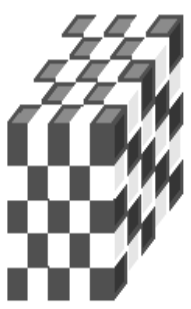

(c)

Fig. 15. Examples of different PBG, (a) 1D, (b) 2D and (c) 3D configurations.
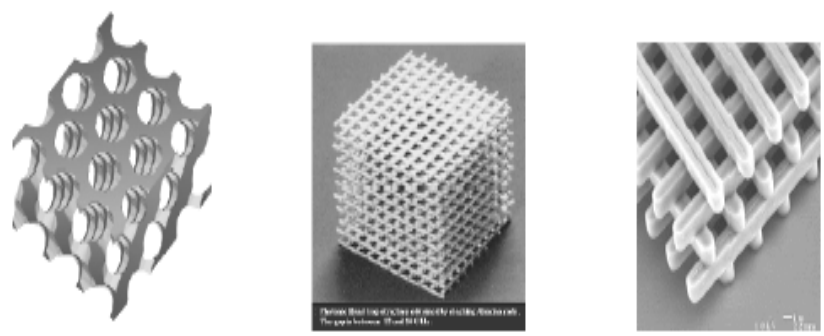

Fig. 16. Typical examples of three-dimensional photonic crystals.

i. The Unit Cell

As stated in the American Heritage Dictionary of the English Language, definition of a crystal (unit cell) is "a homogenous solid formed by a repeating, three-dimensional pattern of atoms, molecules, or shapes, having fixed distances between constituent parts". This replicating pattern is referred to as the unit cell of a crystal. 
Figure 17 illustrates the unit cell for the circular crystal lattice. The unit cell contains all the pertinent information of the crystal such as the crystal geometry (shape, thickness, etc.), material properties (dielectric or magnetic), and the lattice spacing (shown as the dimension " $\mathrm{p}$ ". It is this replicating unit cell that provides the periodicity in the crystal, and controls the location and extent of the band-gap.

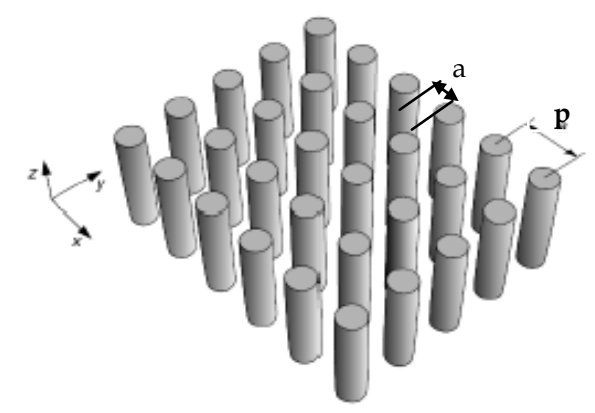

(a)

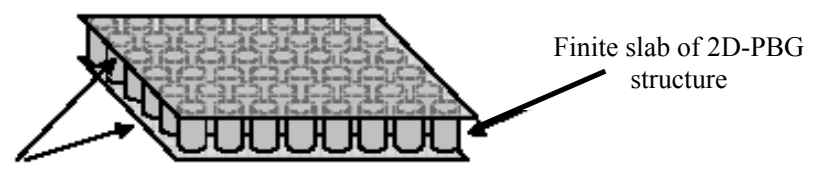

Two PEC plates

(b)

Fig. 17. Practical implementation of 2D PBG structure, (a) Finite slab of 2D PBG structure surrounded by air, (b) Finite slab of 2D PBG structure surrounded by two PEC plates.

ii. The Band-gap

As a first order approximation, a band-gap is obtainable in a high dielectric material with integrated photonic crystals when an incident electromagnetic field propagates with a guide-wavelength, approximately equal to the lattice spacing of the crystal.

$$
\lambda_{\mathrm{r}}=\frac{\mathrm{c}}{\mathrm{f}_{\mathrm{r}} \sqrt{\varepsilon_{\mathrm{r}}}}=2 \mathrm{a}
$$

This rough approximation locates the center of the band-gap, which can extend higher than $\pm 10 \%$ of the center frequency for high dielectric constant materials [67] by using a typical transmission coefficient $\left(\mathrm{S}_{21}\right)$ plot for a 2-port network, such as a microstrip transmission line. This curve, figure19, illustrates that as port 1 of the transmission line is excited, over $15 \mathrm{~dB}$ of attenuation is experienced as the energy propagates from port 1 to port 2 . Thus, the photonic crystal introduces a stop-band filter response. Indeed, the formation of the bandgap is heavily dependent on (1) the periodicity of the crystal, (2) the refractive index (dielectric constant) ratios between the base material (the substrate as a whole) and the impurities that form the crystal. Typically, the refractive index ratio must be at least 2:1 (substrate-to-impurity) ratio for the band-gap to exist. For the 2-D triangular structure, the 
broadest band-gap is obtainable when the impurities (the cylindrical post) are of air $\left(\varepsilon_{\mathrm{r}}=1\right.$ ), while the base material is a high dielectric constant (for example, $\varepsilon_{\mathrm{r}}=10$ ). A 10:1 dielectric $(\sqrt{10}=3.16: 1$ refractive index $)$ ratio would satisfy the index requirement and form a broad band-gap, with proper crystal spacing. This explains the need for a high dielectric substrate for a patch antenna designed on a photonic crystal substrate [68].

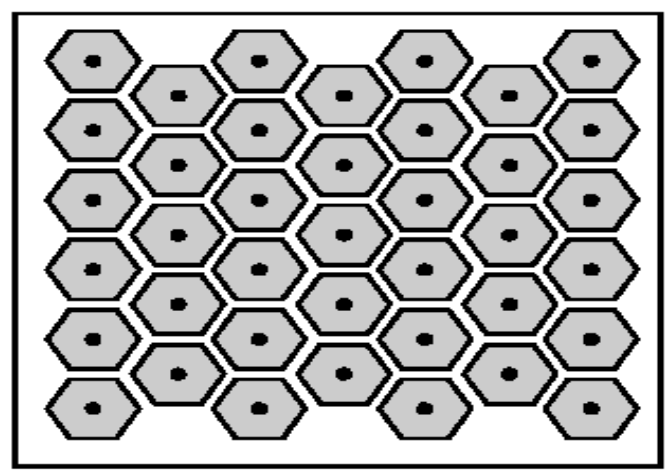

Fig. 18. Top view of the high-impedance surface.

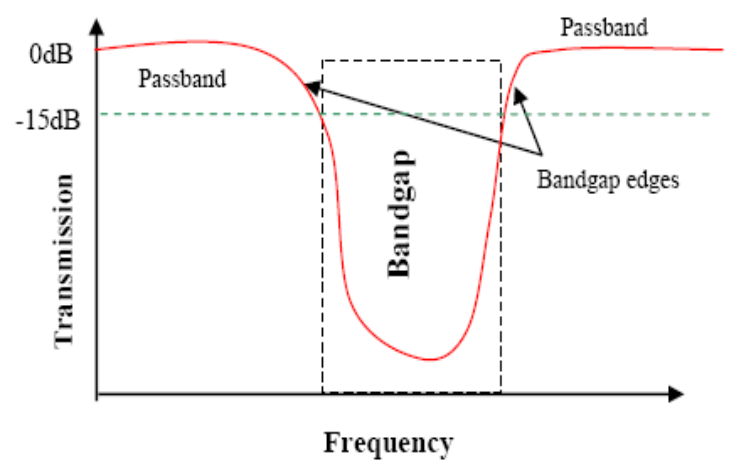

Fig. 19. Transmission loss plot illustrates band gap in a microstrip transmission line at microwave frequencies.

iii. Defects in Periodicity

A photonic crystal essentially behaves much like a band-stop filter, rejecting the propagation of energy over a fixed band of frequencies. However, once a defect is introduced such that it disrupts the periodicity in the crystal, an area to localize or "trap" electromagnetic energy is established. In this region, a pass-band response is created. This ability to confine and guide electromagnetic energy has several practical applications at microwave frequencies as filters, couplers, and especially antennas. This rather simple concept of placing defects in a photonic crystal structure introduces a new methodology in the design of microstrip (patch) antennas. The idea is to design a patch antenna on a $2 \mathrm{D}$ photonic crystal substrate, where the patch becomes the "defect" in the crystal structure. 
In this case, crystal arrays of cylindrical air holes are patterned into the dielectric substrate of the patch antenna. By not patterning the area under the patch, a defect is established in the photonic crystal, localizing the EM fields. Surface waves along the XY plane of the patch are forbidden from forming due to the periodicity of the photonic crystal in that plane. This prevention of surface waves improves operational bandwidth and directivity, while reducing side-lobes and coupling, which are common concerns in microstrip antenna designs [68]. Using these concepts, a photonic crystal patch antenna was developed.

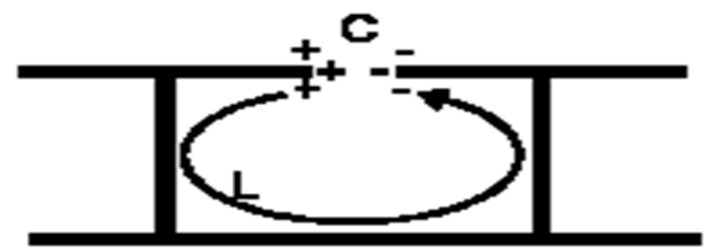

Fig. 20. Origin of the equivalent circuit elements.

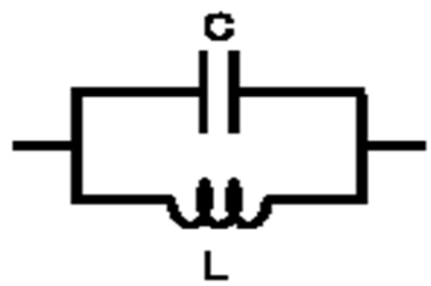

Fig. 21. Equivalent circuit model for the high-impedance surface

\subsection{High Impedance Electromagnetic Surface (HIES)}

\subsubsection{Introduction and background of HIES}

A new type of metallic electromagnetic structure has been developed. It is characterized by high surface impedance. Although it is made of continuous metal and conducting DC currents, it does not conduct AC currents within a forbidden frequency band. Unlike normal conductors, this new surface does not support propagating surface waves, and it reflects electromagnetic waves with no phase reversal. The geometry consists of a metal sheet, textured with a two-dimensional lattice of resonant elements, which act as a twodimensional filter to prevent the propagation of electric currents. The surface can be described using a lumped parameter circuit model, which accurately predicts many of its electromagnetic properties. This unique material is applicable to a variety of electromagnetic problems, including new kinds of low-profile antennas. By incorporating a special texture on a conducting surface, it is possible to alter its radio-frequency electromagnetic properties [69].

In the limit where the period of the surface texture is much smaller than the wavelength, the structure can be described using an effective medium model, and its qualities can be 
summarized into a single parameter: the surface impedance. A high-impedance surface, shown in figure 18, consists of an array of metal, protrusions on a flat metal sheet. They are arranged in a two-dimensional lattice, and are usually formed as metal plates, connected to the continuous lower conductor by vertical posts as shown in figure 20 . They can be visualized as mushrooms or thumbtacks or other shapes protruding from the surface. High Impedance Surfaces as two dimensional EBG structures can be used as microstrip antenna substrate to eliminate the surface wave [70].

\subsubsection{High-impedance surfaces properties}

The properties of the new high-impedance surface are similar to those of the corrugated slab. The quarter-wavelength slots have simply been folded up into lumped elements, capacitors and inductors that are distributed in two dimensions. The two-dimensional array of resonant elements can be explained using a simple circuit model. The capacitance is due to the proximity of the top metal patches, while the inductance originates from current loops within the structure, as shown in figure 20 . The electromagnetic properties of the surface can be predicted by using an equivalent LC circuit, shown in figure 21 . The impedance of a parallel resonant LC circuit, given in Eq. 3, is qualitatively similar to the tangent function that describes the impedance of the corrugated surface.

$$
Z_{s}=\frac{j \omega L}{1-\omega^{2} L C}
$$

It is inductive at low frequencies, and thus supports TM surface waves. It is capacitive at high frequencies, and supports TE surface waves. In a narrow band around the LC resonance, the impedance is very high. In this frequency range, currents on the surface radiate very efficiently, and the structure suppresses the propagation of both types of surface waves. Having high surface impedance, it also reflects external electromagnetic waves without the phase reversal that occurs on a flat conductor. By using lumped elements, we retain the reflection phase and surface wave properties of the quarter-wave corrugated slab, while reducing the overall thickness to a small fraction of a wavelength [7274].

\subsubsection{Improved surface wave current}

Surface waves are excited on microstrip antenna when the substrate $\epsilon_{r}>1$. Besides end fire radiation, surface waves give rise to coupling between various elements of an array. Surface waves are launched into the substrate at an elevation angle $\theta$ lying between $\Pi$ / 2 and $\sin ^{-1}\left(1 / \sqrt{\varepsilon_{\mathrm{r}}}\right)$. These waves are incident on the ground plane at this angle, get the reflected from there, then meet the dielectric-air interface, which also reflect them. Following this zig-zag path, they finally reach the boundaries of the microstrip structure where they are reflected back and diffracted by the edges giving rise to end-fire radiation [94]. On other way in the boundary, if there is any other antenna in proximity, the surface wave can become coupled into it. Surface waves will decay as $1 / \sqrt{\varepsilon_{\mathrm{r}}}$ so that coupling also decreases away from the point of excitation. Surface wave are TM and TE modes of the substrate. These modes are characterized by waves attenuating in the transverse 
direction (normal to the antenna plane) and having a real propagation constant above the cut-off frequency. The phase velocity of the surface waves is strongly dependent on the substrate parameters $h$ and $\epsilon_{\mathrm{r}}$. Figure 22 shows the propagation of the surface wave in microstrip antenna [70].

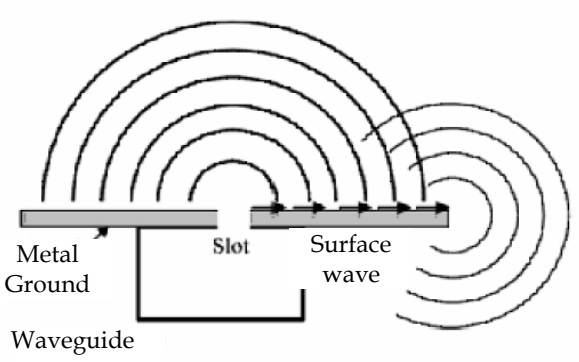

(a)

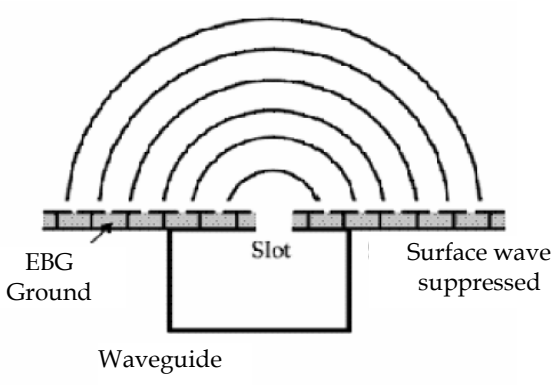

(b)

Fig. 22. (a) The substrate without EBG structure, (b) with EBG structure.

Surface wave propagation is a serious problem in microstrip antennas. It reduces antenna efficiency and gain, limits bandwidth, increases end-fire radiation, increases crosspolarization levels, and limits the applicable frequency range of microstrip antennas. Two solutions to the surface wave problem are available now. One of the approaches is based on the micromachining technology in which part of the substrate beneath the radiating element is removed to realize a low dielectric constant environment for the antenna. In this case the power loss through surface wave excitation is reduced and coupling of power to the space wave is enhanced. The second technique relies on electromagnetic band-gap structure (EBG) engineering. In this case, the substrate is periodically loaded so that the surface wave dispersion diagram presents a forbidden frequency range (stop-band or band-gap) about the antenna operating frequency. Because the surface waves cannot propagate along the substrate, an increase amount of radiating power couples to the space waves. Also, other surface wave coupling effects like mutual coupling between array elements and interference with onboard systems are now absent [43].

\subsubsection{Artificial Magnetic Conductors (AMC)}

Background of AMC

Artificial magnetic conductors (AMC), also known as high-impedance surfaces [76] as shown in figure 23(a) and (b), have received considerable attention in recent years. An AMC is a type of electromagnetic band-gap (EBG) material or artificially engineered material with a magnetic conductor surface for a specified frequency band. AMC structures are typically realized based on periodic dielectric substrates and various metallization patterns. Several types of AMC ground planes have already been extensively studied. AMC surfaces have two important and interesting properties that do not occur in nature and have led to a wide range of microwave circuit applications. 


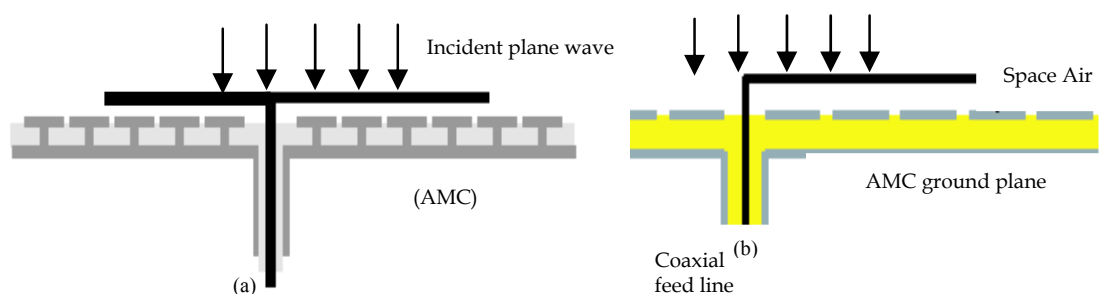

Fig. 23. Horizontal patch antenna on Artificial Magnetic Conductors ground plane.

In the artificial surfaces such as artificial magnetic conductors, the high-impedance surface has proven useful as an antenna ground plane. AMC takes advantage of both the suppression of surface waves and the unusual reflection phase. As a result of the suppression of surface waves, an antenna on Artificial Magnetic Conductors produces a smoother radiation profile than a similar antenna on a conventional metal ground plane, with less power wasted in the backward direction. This can be applied to a variety of antenna designs, including patch antennas, which often suffer from the effects of surface waves. For phased arrays, the suppression of surface waves can reduce inter-element coupling, and help to eliminate blind angles. AMC is particularly applicable to the field of portable hand-held communications, in which the interaction between the antenna and the user can have a significant impact on antenna performance. Using this new ground plane shown in figure 23 as a shield between the antenna and the user in portable communications equipment can lead to higher antenna efficiency, longer battery life, and lower weight.

\begin{tabular}{|c|c|c|c|c|}
\hline Parameter & Dimension Change & $\begin{array}{c}\text { Center } \\
\text { Frequency }\end{array}$ & $\begin{array}{c}\text { Band edge } \\
\text { Effect }\end{array}$ & Bandwidth \\
\hline \multirow[t]{2}{*}{ Effect of $h_{1}$} & $\overline{h_{1}} \downarrow$ & Negative & Lower Side & Positive \\
\hline & & Correlation & Band & Correlation \\
\hline \multirow[t]{2}{*}{ Effect of $\varepsilon_{\mathrm{r}}$} & & Negative & Upper Side & Negative \\
\hline & $\begin{array}{c}\varepsilon_{r} \\
\end{array}$ & Correlation & Band & Correlation \\
\hline \multirow{2}{*}{$\begin{array}{l}\text { Effect of } \\
\text { radius }\end{array}$} & & Negative & Upper Side & Negative \\
\hline & $\rightarrow$ & Correlation & Band & Correlation \\
\hline \multirow{2}{*}{ Effect of gap } & $g$ & Positive & Upper Side & Positive \\
\hline & & Correlation & Band & Correlation \\
\hline Via position & $0^{2 m}$ & $\begin{array}{c}\text { Negative } \\
\text { correlation }\end{array}$ & $\begin{array}{c}\text { Upper side } \\
\text { band }\end{array}$ & $\begin{array}{c}\text { Negative } \\
\text { Correlation }\end{array}$ \\
\hline Via type & $\bar{\square}$ & No Effect & No Effect & No Effect \\
\hline
\end{tabular}

Table 3. Parameters Analysis of EBG Design. 
AMC surfaces have very high surface impedance within a specific limited frequency range, where the tangential magnetic field is small, even with a large electric field along the surface [73]. Therefore, an AMC surface can have a reflection coefficient of +1 (in-phase reflection). Generally, the reflection phase is defined as the phase of the reflected electric field which is normalized to the phase of the incident electric field at the reflecting surface. It can be called in-phase (or out-of-phase) reflection, if the reflection phase is 0 (or not). In practice, the reflection phase of an AMC surface varies continuously from +180 to -180 relative to the frequency, and crosses zero at just one frequency (for one resonant mode). The useful bandwidth of an AMC is generally defined as 135 to 45 on either side of the central frequency. Thus, due to this unusual boundary condition, in contrast to the case of a conventional metal plane, an AMC surface can function as a new type of ground plane for low-profile wire antennas, which is desirable in many wireless communication systems.

\begin{tabular}{|c|c|c|}
\hline Difference & EEBG & UP-EBG \\
\hline Substrate structure & Low Periodic Coplanar PBG \\
\hline Impedance & Low impedance Surface (LIS) & High impedance Surface $(\mathrm{HIS})$ \\
\hline Defacement shapes & & \\
\hline $\begin{array}{l}\text { Equivalent circuit } \\
\text { Resonance } \\
\text { frequency }\end{array}$ & $f_{0}=\frac{1}{2 \pi \sqrt{L\left(C_{1}+C_{2}\right)}}$ & $\Delta \omega / \omega_{0}=\frac{Z_{0}}{\eta}$ \\
\hline Bandwidth & $\pm \Delta \omega=\omega_{0} \mathrm{C}_{1} \frac{\left(1-\mathrm{L}_{1} \mathrm{~L}_{2} \omega_{0}^{2}\right) \mathrm{Z}_{0}}{4 \mathrm{C}}$ & \\
\hline
\end{tabular}

Table 4. Comparison between different characteristics of electrical equivalent circuit for both embedded EBG and UP- EBG.

\section{Design of single patch antennas with DGS}

\subsection{Miniaturized design consideration}

A novel design method using the first iteration of fractal carpet gasket is utilized as DGS cell to study the size miniaturization. Figure 24(a) shows schematics of the proposed DGS cell. The DGS is etched on the back side of the metallic ground plane as shown in figure 24(b). The substrate is RT/Duroid with $0.813 \mathrm{~mm}$ thickness and dielectric constant $\varepsilon_{\mathrm{r}}=3.38$. The feed line width $w=1.85 \mathrm{~mm}$ is chosen for the characteristics impendence of $50 \Omega$ microstrip line at frequency $5.25 \mathrm{GHz}$. The defect unit is with length $\mathrm{L}=12 \mathrm{~mm}$ and width $\mathrm{W}=9 \mathrm{~mm}, \mathrm{Lg}_{\mathrm{g}}=1 \mathrm{~mm}$, $L_{\mathrm{s}}=1 \mathrm{~mm}, \mathrm{~W}_{\mathrm{g}}=1 \mathrm{~mm}$ and $\mathrm{W}_{\mathrm{s}}=3 \mathrm{~mm}$. The effect of one as well as two unit cells of rectangular DGS (RDGS) on inset feed MPA performance is studied by etching the defect from certain reference point as a start at distance $X=0 \mathrm{~mm}$ as shown in figure 24(b) and gradually increase $\mathbf{X}$ to change the position of DGS with step 2mm until DGS is far away from the image projection 
under the radiating surface. For further reduction two unit cells of the proposed RDGS are used, which are etched in face to face with separation $3 \mathrm{~mm}$. it is noted that the maximum size reduction is $35 \%$ in case of one unit cell at defected displacement $\mathbf{X}=10 \mathrm{~mm}$ from antenna edge. In case of two unit cells the maximum reduction ratio reached $53 \%$ at same defect position [82].

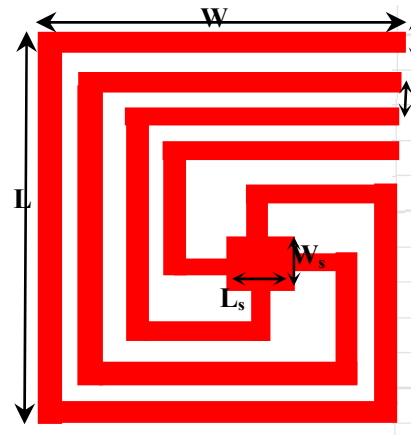

(a)

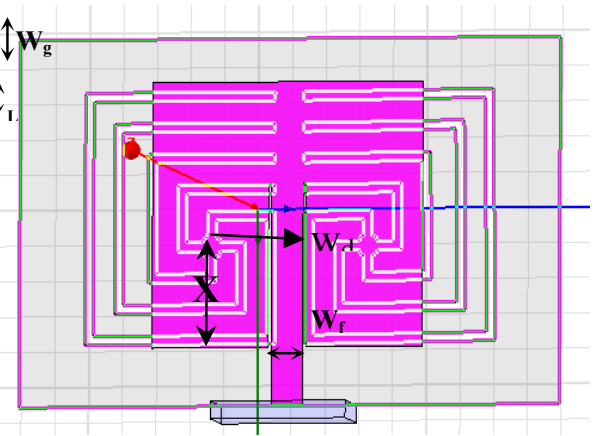

(b)

Fig. 24. (a) The geometry of one unit cell of DGS, (b) the proposed antenna prototype

\subsection{Multiband design consideration}

In many applications it is also important to design single feed antenna for multiband resonant frequencies. Spiral defected ground structure DGS is used to provide both multi resonant frequencies and compact size. Figure 24(a) shows schematic of the proposed one cell spiral DGS. Spiral structures, however, are known to produce large cross polarization. Therefore to completely eliminate the cross polarization, a four-arm spiral is explored, as shown in figure $24(\mathrm{~b})$. Four spiral branches, each with a $0.01 \lambda_{5.2 \mathrm{GHz}}$ width, split from the center and rotate outwards are used in the design.

As it may be seen, this geometry is symmetric not only in $+/-\mathrm{x}$ or $+/-\mathrm{y}$ directions, but also in $x$ and $y$ directions. A design using one unit cell of the spiral DGS is simulated and it is clear that the effect of increasing distance $\boldsymbol{X}$ on antenna performance of one cell as well as two unit cells. As distance $\mathbf{X}$ increases the resonant frequencies are reduced until certain distance and after that the trend is reversed. Maximum reduction in the fundamental resonant frequency was achieved at distance $X=9 \mathrm{~mm}\left(0.16 \lambda_{5.2 \mathrm{GHz}}\right)$ and DGS shifted from width center $\boldsymbol{W}_{\boldsymbol{d}}$ by $3 \mathrm{~mm}\left(0.083 \lambda_{5.2 \mathrm{GHz}}\right)$. More reduction in resonant frequency was achieved by using two unit cells of spiral DGS which are placed face to face and at a separation distance from center to center $2 \boldsymbol{W}_{\boldsymbol{d}}$ equal to $10 \mathrm{~mm}\left(0.18 \lambda_{0}\right)$ with separation $\mathbf{W}_{\mathrm{g}}$ equal to $3 \mathrm{~mm}$ $\left(0.054 \lambda_{5.2 \mathrm{GHz}}\right)$. We use same previous substrate and antenna dimensions are $15 \times 16 \mathrm{~mm}^{2}$ and DGS is etched in the bottom of the metallic ground plane as in figure 24 (b). The dimension of the largest arms length $\mathrm{L}=13.25 \mathrm{~mm}$ and largest width $\mathrm{W}=11.25 \mathrm{~mm}$, air-gap $g=0.5 \mathrm{~mm}$ and $L_{g}=2 \mathrm{~mm}$ with spacing patch $L_{s}=W_{s}=1 \mathrm{~mm}$ with $0.5 \mathrm{~mm}$ inner square is used in the design. The line width $W_{f}=1.85 \mathrm{~mm}$ is chosen for the characteristics impendence of $50 \Omega$ microstrip line at frequency $5.25 \mathrm{GHz}$. The fundamental MPA resonance frequency is reduced by using one unit cell spiral DGS which is shifted from length center by $0.011 \lambda_{0}$. In case of using two unit cells, maximum reduction in resonant frequency occurs when the cells are face to face and centered under radiating plate but far from width center by $0.05 \lambda_{0}$. 


\subsection{Simulation and measurement of miniaturized multi-band antenna}

The final two proposed antenna configurations were fabricated as shown in figure 25 by using photolithographic techniques. The reduction in antenna resonant frequency is due to increase in both electrical and magnetic coupling from ground to radiating antenna plate. The measured and simulated results are shown in figure 26. Second proposed antenna was fabricated also and the multi-band operation and reducing antenna size was achieved and the comparison between simulation and measurement of reflection coefficient is shown in figure 27 [81].

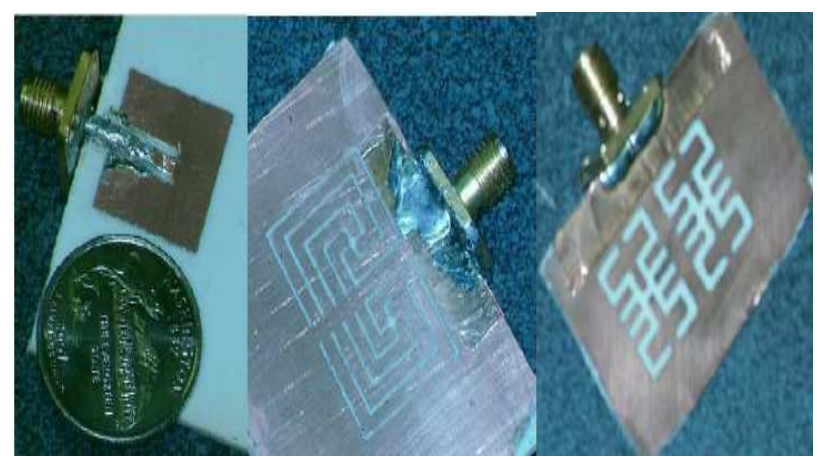

Fig. 25. Photo of the fabricated two antennas.

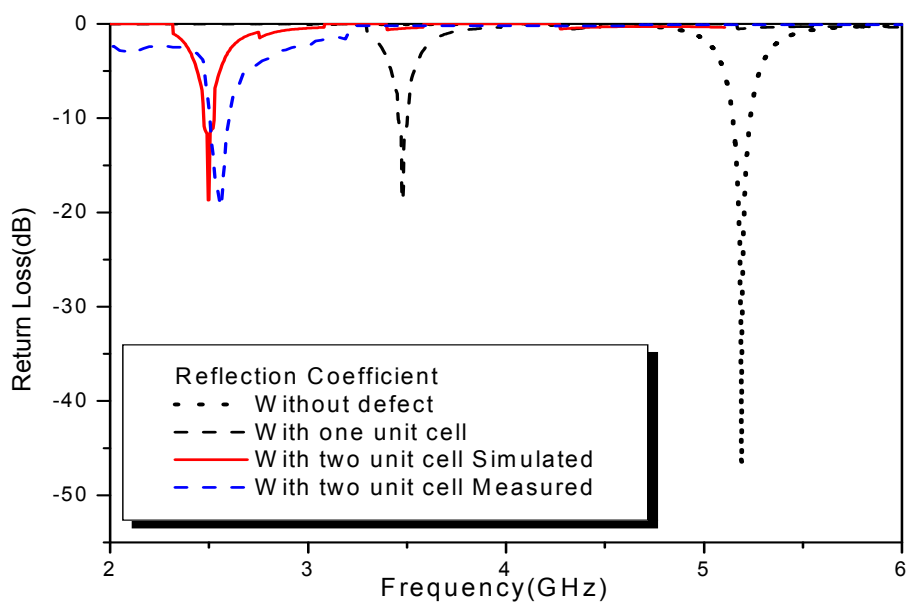

Fig. 26. Comparison between measured and simulated reflection coefficient of antenna without and with one and two gasket unit cell 


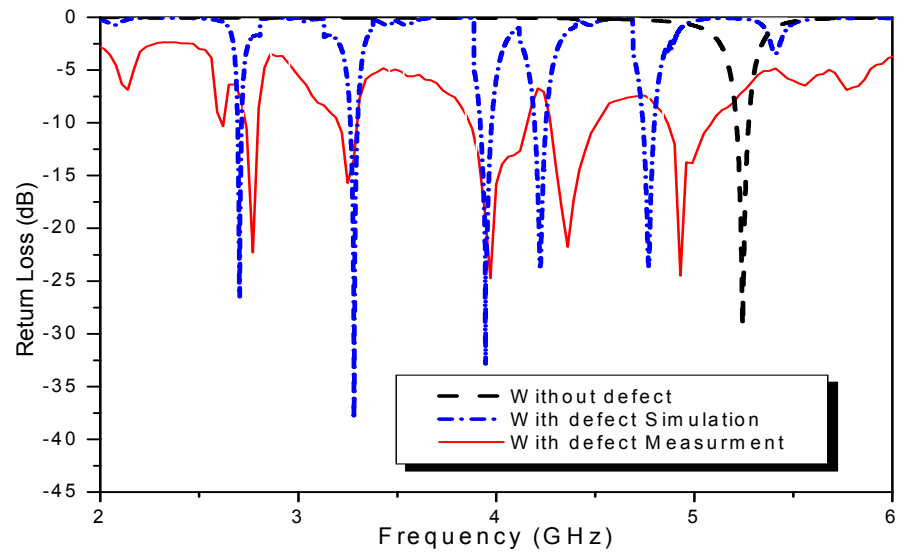

Fig. 27. Comparison between measured and simulated reflection coefficient of antenna without and with two unit cell of spiral DGS simulated and measured.

\section{Design of single patch antennas with EBG}

The conventional half-wavelength size is relatively large in modern portable communication devices. Various approaches have been proposed, such as using shorting pins, cutting slots, and designing meandering microstrip lines. Increasing the dielectric constant of the substrate is also a simple and effective way in reducing the antenna size. Applications of MPAs on high dielectric constant substrate are of growing interest due to their compact size and conformability with monolithic microwave integrated circuits (MMIC). However, there are several drawbacks with the use of high dielectric constant substrate, namely, narrow bandwidth, low radiation efficiency, and poor radiation patterns, which result from strong surface waves excited in the substrate. The narrow bandwidth can be expanded by increasing the substrate thickness, which, however, will launch stronger surface waves. As a result, the radiation efficiency and patterns of the antenna are further degraded. To quantify this phenomenon, a comparative study of MPAs on substrates with different dielectric constants and different thicknesses is performed in this section. Table 5 illustrates the four samples under study. Two of them with low dielectric constant substrate $\left(\varepsilon_{\mathrm{r}}=2.2\right)$ and the other two are built on the high dielectric constant substrate $\left(\varepsilon_{\mathrm{r}}=10.2\right)$.

\begin{tabular}{|c|c|c|c|}
\hline Example & Patch size $\mathbf{~ m m}^{\mathbf{2}}$ & Dilectric constant $\left(\varepsilon_{\mathbf{r}}\right)$ & Height $(\mathbf{m m})$ \\
\hline 1 & $18 \times 10$ & 2.2 & 1 \\
\hline 2 & $16 \times 13$ & 2.2 & 2 \\
\hline 3 & $9 \times 6$ & 10.2 & 1 \\
\hline 4 & $8 \times 6$ & 10.2 & 2 \\
\hline
\end{tabular}

Table 5. The antennas parameters. 
Figure 28 shows the simulated $S_{11}$ of these four structures. By tuning the patch size and the feeding probe location, all the antennas match well to $50 \Omega$ around $5.1 \mathrm{GHz}$. It is noticed that the patch sizes on high dielectric constant substrate are remarkably smaller than those on low dielectric constant substrate as shown in table 5, which is the main advantage of using high dielectric constant substrate. However, the antenna bandwidth $\left(S_{11}<10 \mathrm{~dB}\right)$ on $1 \mathrm{~mm}$ substrate height is decreased from $1.38 \%$ to $0.61 \%$ when the $\varepsilon_{\mathrm{r}}$ is increased from 2.2 to 10.2. Similar phenomenon is observed for the $2 \mathrm{~mm}$ height, the bandwidth is decreased from $2.40 \%$ to $1.71 \%$. For the same dielectric constant substrates, the antenna bandwidth is enhanced when the thickness is doubled. For example, the antenna bandwidth on the high dielectric constant substrate is increased from $0.61 \%$ to $1.71 \%$ when the substrate thickness is increased from $1 \mathrm{~mm}$ to $2 \mathrm{~mm}$. It's important to point out that the bandwidth of example (4) is even larger than that of example (1), which means that the bandwidth of MPAs on high permittivity substrate can be recovered by increasing the substrate thickness.

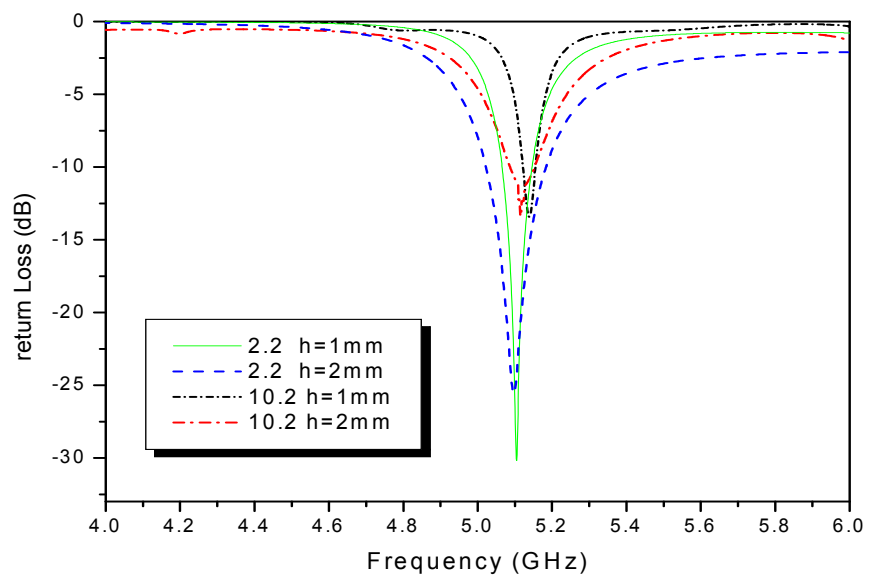

Fig. 28. Return loss comparison of patch antennas with different dielectric constants and substrate height.

Figure 29 compares the H-plane radiation patterns of these four antennas. A finite ground plane of $\lambda \times \lambda$ size is used in the simulations, where $\lambda$ is the free space wavelength at 5.1 GHz. The antennas on the high dielectric constant substrates exhibit lower directivities and higher back radiation lobes than those on the low dielectric substrates. For antennas on the same dielectric constant substrate, when the thickness increases, the antenna directivity decreases, especially for those on high dielectric constant substrates. Similar observations are also found in the E-plane patterns.

These phenomena can be explained from the excitation of surface waves in the substrate. When a high dielectric constant and thick substrate is used, strong surface waves are excited. This causes reduction of the radiation efficiency and directivity. In addition, when the surface waves diffract at the edges of the ground plane, the back radiation is typically increases. 


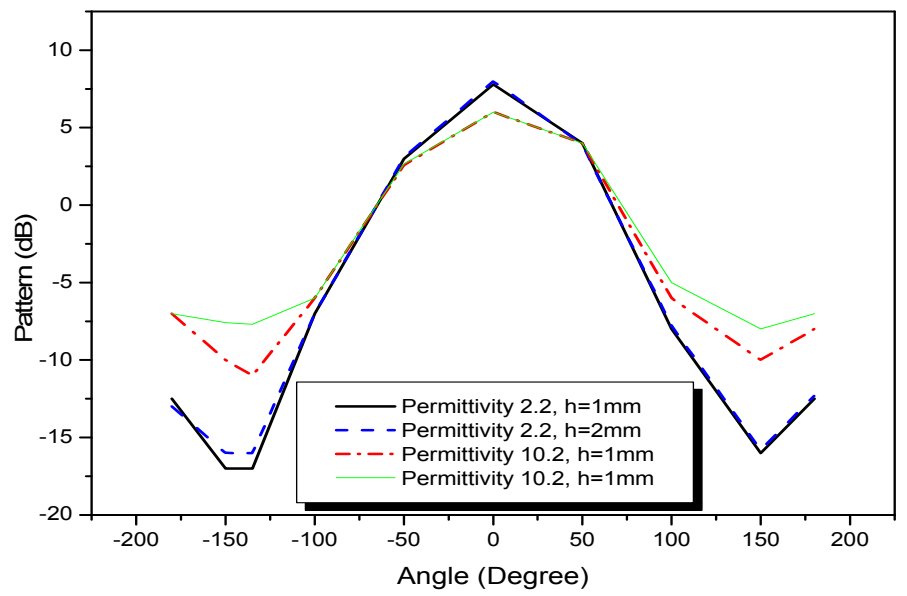

Fig. 29. H-Plane radiation pattern of patch antennas with different dielectric constants and substrate heights.

\section{i. Gain Enhancement of a Single Patch Antenna}

To overcome the drawbacks of using the thick and high dielectric constant substrate, several methods have been proposed to manipulate the antenna substrate. One approach suggested is to lower the effective dielectric constant of the substrate under the patch using micromachining techniques [94]. A shortcoming of this approach is the larger patch size than that on an unperturbed substrate. Another approach is to surround the patch with a complete band gap structure or synthesized low dielectric constant substrate so that the surface wave's impact can be reduced. A MPA design is proposed that does not excite surface waves. In this section, EBG structure is applied in patch antenna design to overcome the undesirable features of the high dielectric constant substrates while maintaining the desirable features of utilizing small antenna size.

\section{ii. Patch Antenna Surrounded by EBG Structures}

Figure 30 sketches the geometry of a MPA surrounded by a mushroom-like electromagnetic band gap (EBG) structure. The EBG is designed so that its surface wave band gap covers the antenna resonant frequency. As a result, the surface waves excited by the patch antenna are inhibited from propagation by the EBG structure. To effectively suppress the surface waves, four rows of EBG cells are used in the design. It is worthwhile to point out that the EBG cell is very compact because of the high dielectric constant and the thick substrate employed. Therefore, the ground plane size can remain small, such as $1 \lambda \times 1 \lambda$. For comparison, MPA designed on a step-like substrate is investigated, as shown in figure 31 . The idea is to use a thick substrate under the patch to keep the antenna bandwidth and use a thin substrate around the patch which reduces the surface waves. The distance between the patch and the step needs to be carefully chosen. If the distance is too small, the resonant feature of the patch will change and the bandwidth will decrease. However, when the distance is too 
large, it cannot reduce the surface waves effectively. To validate the above design concepts, four antennas were simulated on RT/Duroid $6010\left(\varepsilon_{\mathrm{r}}=10.2\right)$ substrate with a finite ground plane of $52 \times 52 \mathrm{~mm}^{2}$. Two of them are normal patch antennas built on $1.27 \mathrm{~mm}$ and 2.54 $\mathrm{mm}$ thick substrates as references. The step-like structure stacks two $1.27 \mathrm{~mm}$ thick substrates under the patch and the distance from the patch edge to the step is $10 \mathrm{~mm}$. The EBG structure is built on $2.54 \mathrm{~mm}$ height substrate and the EBG patch size is $2.5 \times 2.5 \mathrm{~mm}^{2}$ with $0.5 \mathrm{~mm}$ separation. Figure 32 compares the measured $S_{11}$ results of these four antennas. All the four patches are tuned to resonate at the same frequency $5.1 \mathrm{GHz}$. It is noticed that the patch on the thin substrate has the narrowest bandwidth of only $1 \%$ while the other three have similar bandwidths of about $3-4 \%$. Thus, the thickness of the substrate under the patch is the main factor determining the impedance bandwidth of the antenna. The step substrate and the EBG structure, which are located away from the patch antenna, have less effect on the antenna bandwidth.

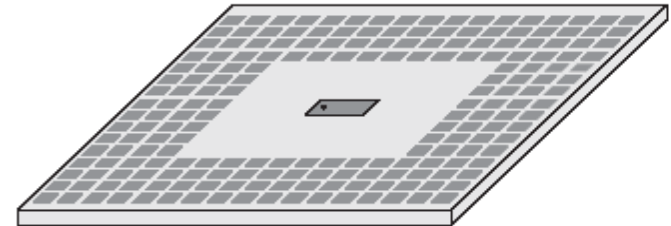

(a)

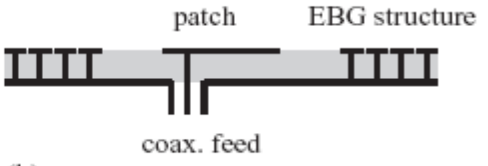

(b)

Fig. 30. Patch antenna surrounded by a mushroom-like EBG structure: (a) geometry and (b) cross section.

The antenna on the height $2.5 \mathrm{~mm}$ has the lowest front radiation while its back radiation is the largest. When the substrate thickness is reduced, the surface waves become weaker and the radiation pattern improves. The step-like structure exhibits similar radiation performance as the antenna on the thin substrate. The best radiation performance is achieved by the EBG antenna structure. Due to successful suppression of surface waves, its front radiation is the highest, which is about $3.2 \mathrm{~dB}$ higher than the thick case. Since the surface wave diffraction at the edges of the ground plane is suppressed, the EBG antenna has a very low back lobe, which is more than $10 \mathrm{~dB}$ lower than other cases. Table 6 lists the simulated results of these antennas. Note that the radiation patterns are normalized to the maximum value of the EBG antenna.

\begin{tabular}{|c|c|c|c|}
\hline Antenna & Bandwidth $\%$ & Front Radiation (dB) & Back Radiation (dB) \\
\hline Thin & 1 & -2.3 & -15.5 \\
Thick & 4 & -3.2 & -12 \\
Step stair & 4.7 & -2 & -14 \\
EBG & 3 & 0 & -25 \\
\hline
\end{tabular}

Table 6. Simulated performance of four different MPA designs on the high dielectric constant substrate. 


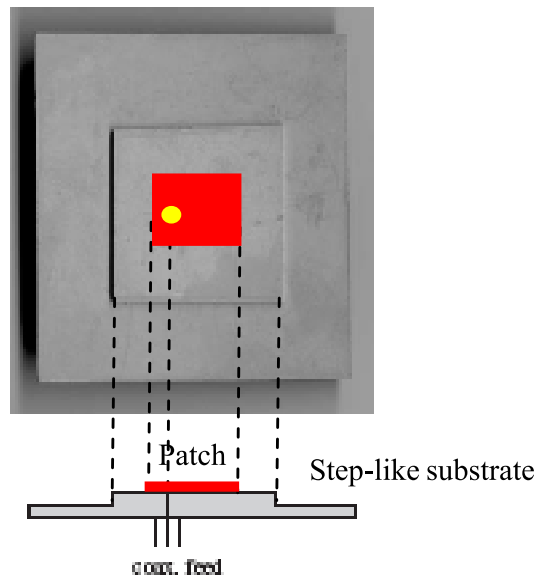

Fig. 31. Patch antenna on a step-like substrate.

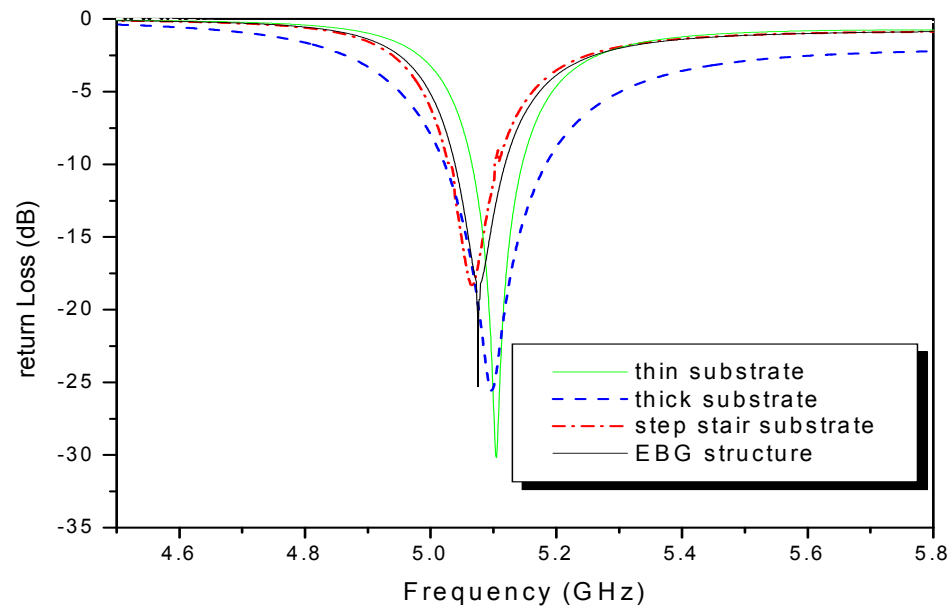

Fig. 32. Comparison of the measured return loss of the four MPA structures.

It is also interesting to notice that in the E-plane the beam-width of the EBG case is much narrower than the other three cases whereas in the H-plane it is similar to other designs. The reason is that the surface waves are mainly propagating along the E-plane as shown in figure 33. Once the EBG structure stops the surface wave propagation, the beam becomes much narrower in the E-plane. From above comparisons it is clear that the EBG structure improves the radiation performances of the patch antenna while maintaining its compact size and adequate bandwidth. 

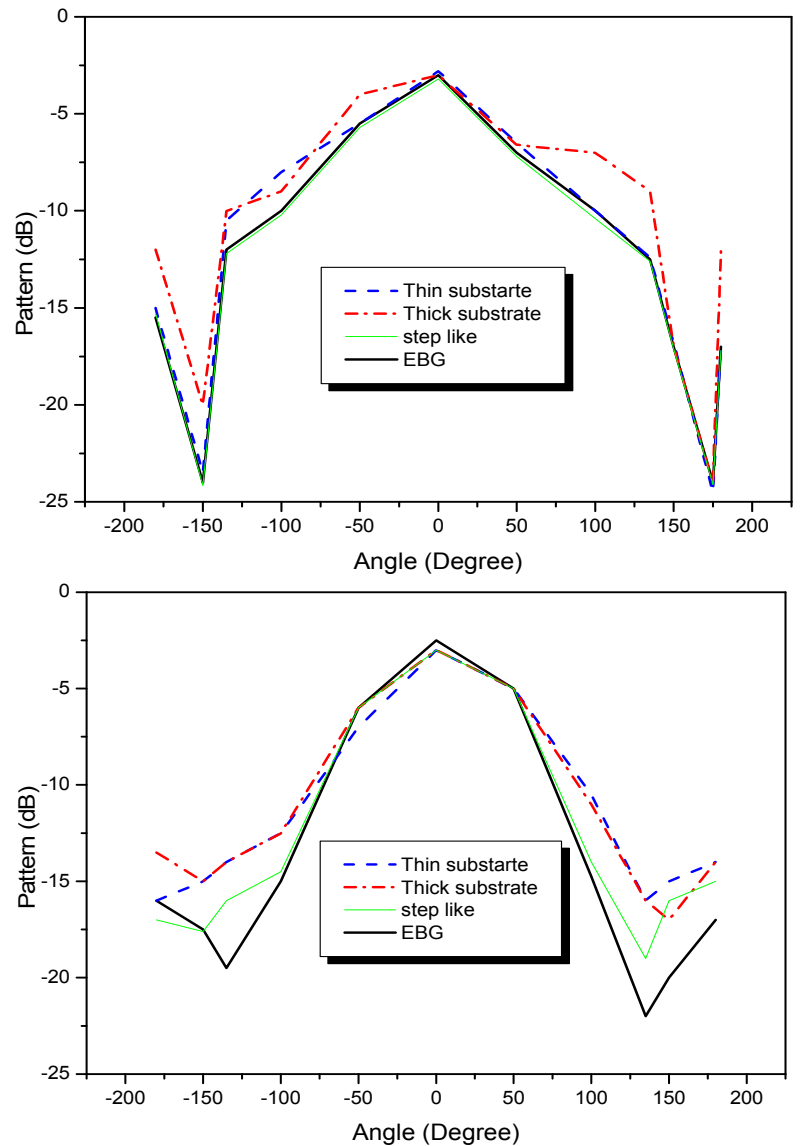

Fig. 33. Simulated radiation patterns of different patch antennas: (a) E-plane and (b) H-plane pattern.

\section{Antenna design for ultra-wideand wireless communications applications}

\subsection{Enhancement of microstrip monopole antenna bandwidth by using EBG structures}

The low profile, light weight, and low cost of manufacturing of monopole microstrip patch antennas have made them attractive candidates in many applications ranging from very high-data rate and short-range wireless communication systems, to modern radar systems. The limited bandwidth of the MPA, however, needs to be further improved to facilitate applications in UWB systems. Spiral arms shaped metallo EBG was used to increase the bandwidth of planar monopole antennas. This approach resulted in size reduction and cceptable performance from 1 to $35 \mathrm{GHz}$. In this section, we present a new approach based on using a variety of shapes and sizes of embedded EBG structures. The designs of the EBG structures as well as the improvement steps in the antenna performance are described in the following sections [89]. 
i.Antenna Geometry and Design A low profile microstrip monopole patch antenna is proposed as shown in figure 34 . Rectangular and circular geometries were simulated to optimize the performance.

Starting with a rectangular patch antenna on a rectangular ground plane and ended up with an umbrella semi-circular shape, for both the radiator and the ground plane. A semi-circular ground plan with radius $\mathrm{R}_{\mathrm{g}}=15 \mathrm{~mm}$ was used as shown in figure 34 . As a last step, circular shapes for both the radiator and the ground plane with radii of $R_{r}=12 \mathrm{~mm}$ for the antenna and $R_{\mathrm{g}}=15 \mathrm{~mm}$ for the ground plane are used as shown in figure 34 . The microstrip feed line length is $L_{f}=16 \mathrm{~mm}$, and width $W_{f}=1.9 \mathrm{~mm}$. The antenna is printed on FR4 substrate with $\varepsilon_{\mathrm{r}}=4.7$ and thickness $3.2 \mathrm{~mm}\left(0.034 \lambda_{\circ}\right)$. After optimizing the basic antenna design with circular radiator and ground plane, various designs of EBG structures are embedded to improve the bandwidth, enhance gain, and reduce the antenna size beyond that was previously reported. The proposed metallic patch antenna layout was first surrounded by an EBG lattice with circular holes with dimension computed as described in [51], hence is given by $\mathrm{d}=\lambda_{\mathrm{g}}$ where $\mathrm{d}$ is the diameter of drilled hole and $\lambda_{\mathrm{g}}$ is the guided wavelength. Then embedded circular and square patches in a periodical electromagnetic band-gap structure were used as shown in figure 34, and the side view is shown in figure 34 . The periodic patches have radius $=3 \mathrm{~mm}$ for the circular patch and, half-square side $=3 \mathrm{~mm}$ for the square patch. The periodicity in the two cases is $P=7 \mathrm{~mm}$, and the vias radius equals $0.25 \mathrm{~mm}$. These dimensions were used based on optimized simulation results. The optimization process includes embedded circular and square patches dimensions as well as antenna parameters such as gain, bandwidth, and radiation efficiency. The fabricated antenna is shown in figure 34 .

\section{ii. Simulation and Experimental Results}

The simulation results are shown in figure 35 (a). Then partial rectangular ground plane is used with dimensions $30 \times 15 \mathrm{~mm}^{2}$. Results for this case are also shown in figure 35 (a). The partial rectangular ground plane is then converted to half circular plane to increase the bandwidth by creating adjacent staggered resonance modes. Both the radiator and the ground plane shapes are then converted to half circular shapes with radii of $12 \mathrm{~mm}$ and $15 \mathrm{~mm}$, respectively. The final design for the basic antenna shows ultra wideband characteristics shown in figure 35 (b) but with bandwidth discontinuity from 7 to $10 \mathrm{GHz}$ and 12.5 to $17.5 \mathrm{GHz}$. To remove these discontinuities, EBG structures are embedded. The effect of EBG on the antenna performance is examined first by drilling cylindrical holes each with a radius equals to $3 \mathrm{~mm}$ and periodicity $\mathrm{P}=7 \mathrm{~mm}$. Then circular and square EBG patches are embedded in the substrate. Simulation results to illustrate the performance of each of the EBG structures (circular or square) as well as the case of the drilled holes are shown in figure 37. These results were obtained by calculating the transmission coefficient of a $50 \Omega$ microstrip transmission line placed on the top of a substrate with either square or circular EBG structure or circular holes. From figure 37, it is seen that each of these embedded structures has different effect on the bandwidth of the microstrip line. The pass-band and stop-band for the embedded square are larger and deeper than that of circular embedded EBG. Embedded circular patch of radius $3 \mathrm{~mm}$ and square patch of side length $d=6 \mathrm{~mm}$ EBG structures with periodicity $\mathrm{P}=7 \mathrm{~mm}$ are combined with the half circular-monopole antenna 
as illustrated in figure 45(a) and 45(b), respectively. The comparison between simulation and measurement of reflection coefficient $S_{11}$ of both embedded EBG are shown in figures 37 and 38 , respectively. From these figures it is noted that not only improvement in the bandwidth was achieved but also the antenna size was reduced to about $34 \%$ from that of the basic half circular monopole antenna without EBG. This gives more than $60 \%$ size reduction higher than that published in [92] by $10 \%$. The final antenna with square embedded patches has average antenna gain of about $6.5 \mathrm{dBi}$. The gain value changes throughout the operating band as shown in figure 38. At lower frequencies, the gain is about $3 \mathrm{dBi}$ and started to increase with frequency until it reaches its maximum value of $8.5 \mathrm{dBi}$ at $12.5 \mathrm{GHz}$. After this, it starts to decrease again with frequency. However, the antenna gain with EBG is larger than without EBG throughout the entire frequency band by an average value of about $2 \mathrm{dBi}$. The antenna radiation efficiency has the highest value from 1 to $7.5 \mathrm{GHz}$ and then it stays between $60-70 \%$ up to $35 \mathrm{GHz}$. The final antenna design has an average efficiency of $73.5 \%$ as compared to only $56.5 \%$ for the basic antenna without embedding EBG structures.

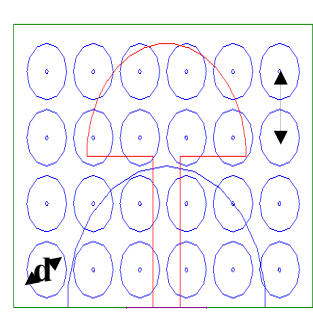

a

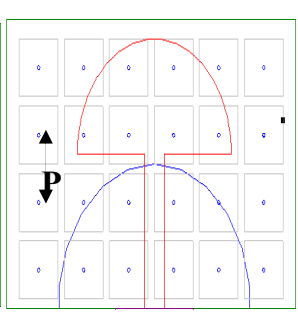

b

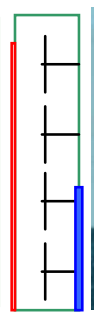

C

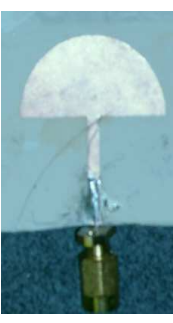

$\mathrm{d}$

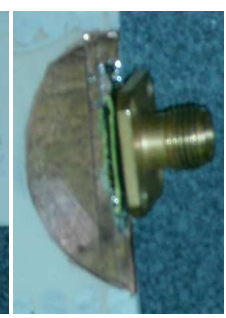

$\mathrm{e}$

Fig. 34. The proposed antenna with embedded EBG (a) Circular EBG, (b) Square EBG (c) side view (d) fabricated radiator monopole antenna, and (e) ground plane on back of the substrate.

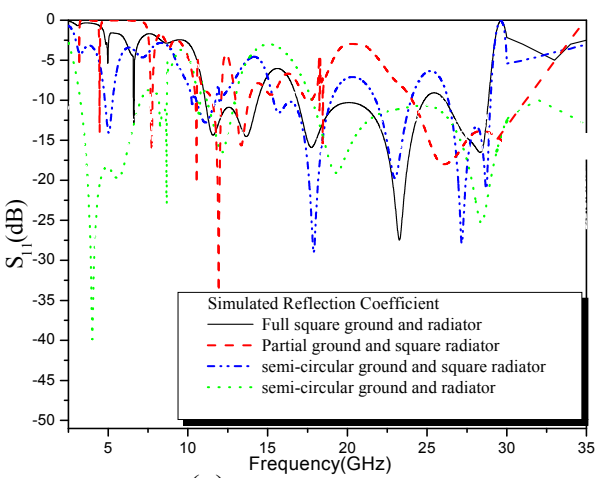

(a)

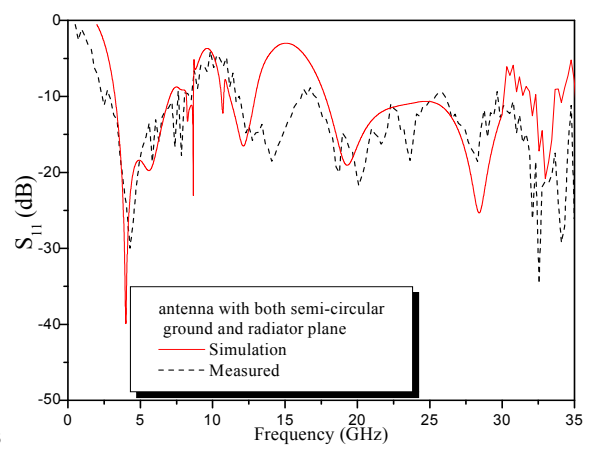

(b)

Fig. 35. (a) Simulated $S_{11}$ vs. frequency for various design parameters and (b) comparison between simulation and measurement for the proposed. 


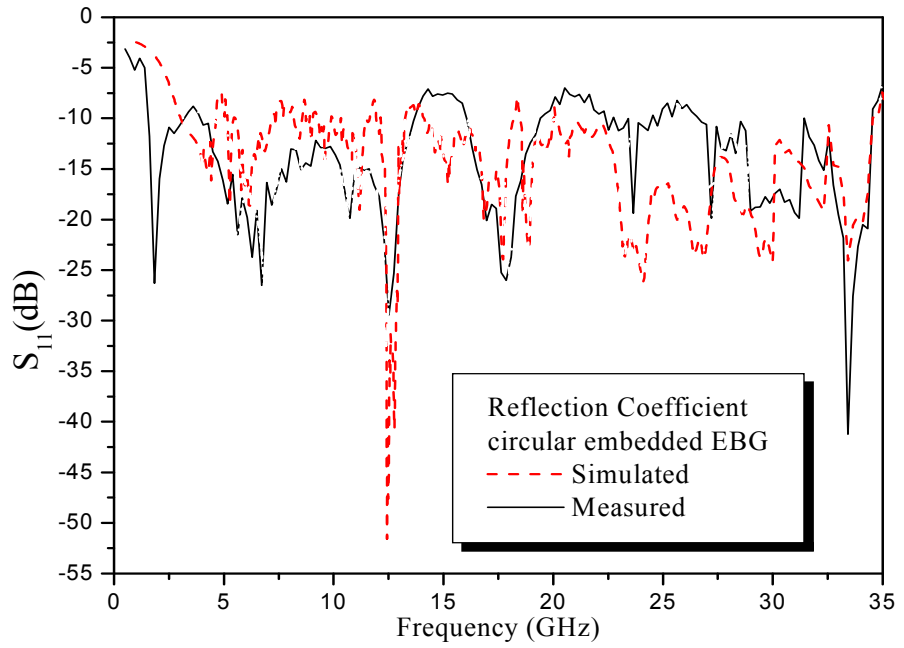

Fig. 36. Comparison between measured and simulated reflection coefficient for umbrella monopole antenna with embedded circular EBG patches.

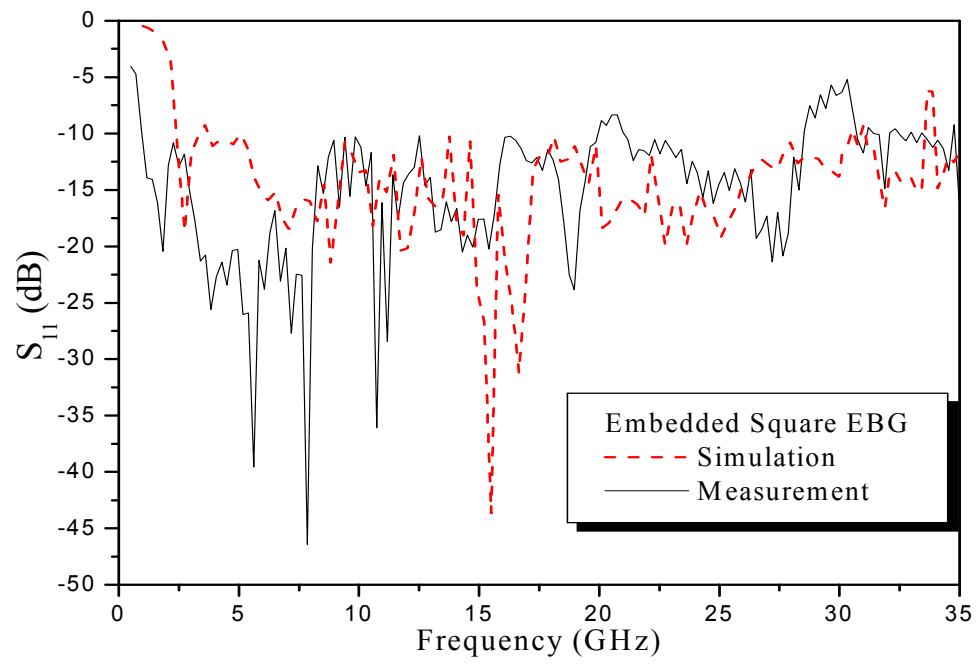

Fig. 37. Comparison between measured and simulated reflection coefficients of umbrella monopole antenna with embedded square EBG patches. 


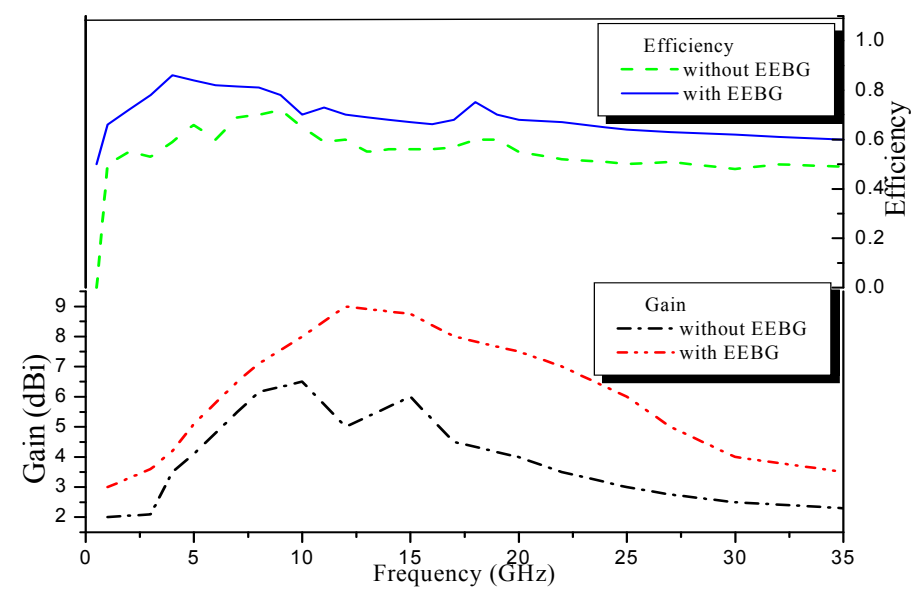

Fig. 38. Antenna gain and radiation efficiency vs. frequency for antennas with and without square EEBG. EBG structure

\subsection{Ultra-wide bandwidth umbrella shaped MMPA using Spiral Artificial Magnetic Conductor (SAMC)}

i. Proposed Antenna Geometry and Design

The proposed antenna has semi-circular shape. The thickness of the substrate used is approximately $0.034 \lambda_{3.3 \mathrm{GHz}}=(3.2 \mathrm{~mm})$. The semi-circular patch of radius $\mathrm{R}_{\mathrm{r}} \mathrm{mm}$, is placed on one side of dielectric substrate with relative permittivity 4.7 and $\tan \delta=0.02$. The dimension for the substrate is $L_{s} \times W_{s} \mathrm{~mm}^{2}$. The antenna is fed by $50 \Omega$ microstrip feed-line of width ' $W_{\mathrm{f}}$ '. The semi-circular radiator is placed ' $\mathrm{L}_{\mathrm{f}}$ ' distance from one edge of the substrate. The design of the proposed antenna started from conventional shape of printed rectangular microstrip monopole. To improve the bandwidth, the radiator was modified to be semicircular patch with radius $12 \mathrm{~mm}$. The antenna size is thus reduced by $22 \%$ from the original size. The dimension of FR4 substrate is $40 \times 40 \mathrm{~mm}^{2}$ but in this case discontinuities in bandwidth were found between frequency band from $1 \mathrm{GHz}$ to $5 \mathrm{GHz}$ and from $6 \mathrm{GHz}$ to $10 \mathrm{GHz}$ as shown in following sections.

\section{ii. Spiral AMC Design}

In this section, two typical printed spiral geometries are investigated as shown in figure 39. The operation principle of the AMC surface can be simply explained by an equivalent LC circuit theory. To increase the value of the inductance, a single spiral is placed on top of the grounded substrate to replace the conventional ground plane. The parameters of the substrate remain the same as the reference conventional monopole. The width of the spiral is $1 \mathrm{~mm}=0.011 \lambda_{3.3 \mathrm{GHz}}$ with gap $=1 \mathrm{~mm}$. Two shapes of spiral AMC are used in the proposed design, one arm spiral as in figure 40(a) and four arms spiral as in figure 40(b). The two spiral shapes are applied on the antenna ground plane as shown in figure 40 (a) 
and $40(\mathrm{~b})$ to improve the performance such as decreasing the antenna size, reducing the bandwidth discontinuities and increasing the antenna gain. After achieving the best possible results from this approach, holes were drilled to further improve the bandwidth and enhance antenna gain as shown in figure 40(c). The design of the drilled holes EBG structure is straight forward, hence, $\mathrm{d}=\lambda_{\mathrm{g}}$ where $d$ is the diameter of drilled holes and $\lambda_{g}$ is the guided wavelength. $\mathrm{d}=1 \mathrm{~mm}$ and a periodicity $=3 \mathrm{~mm}$ were chosen and the fabricated antenna is shown in figure 41(a) and 41(b). The diameter of the drilled holes $d$ is chosen to be sufficiently applied at the desired discontinuities frequencies from 6 to $10 \mathrm{GHz}$. This makes the presence of the holes too small to affect the dielectric constant of the substrate.
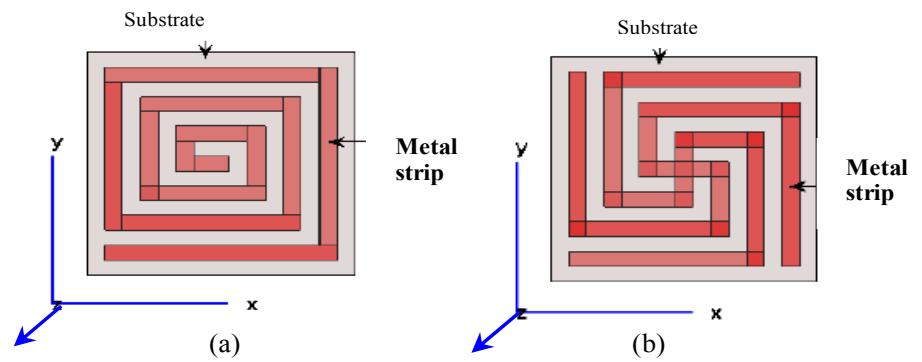

Fig. 39. The spiral shape as AMC; (a) one arm, (b) four arms.

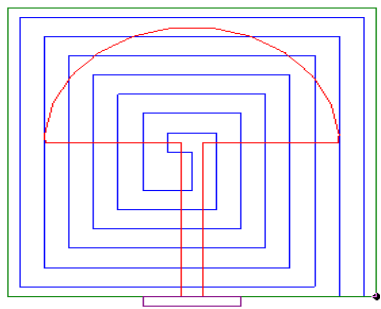

(a)

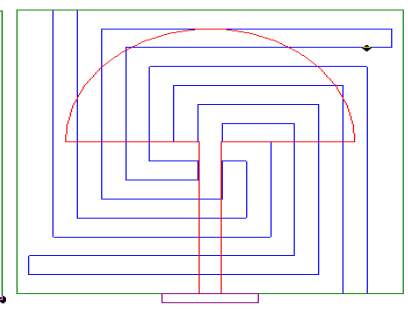

(b)

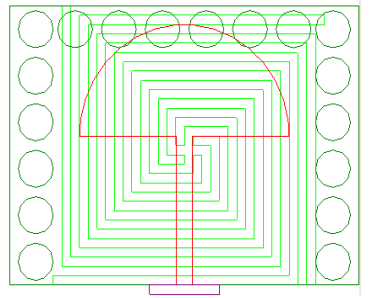

(c)

Fig. 40. Umbrella shape with spiral ground plane (a) one arm (b) four arms and (c) four arms with surrounded holes.

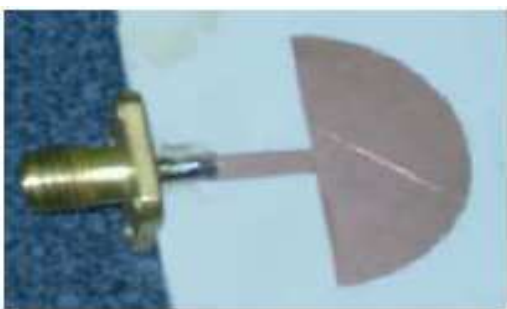

(a) Antenna radiator plane

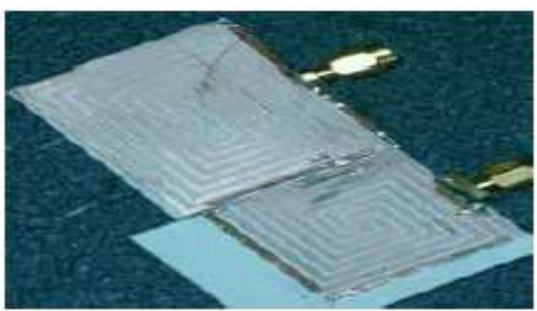

(b) Spiral ground plane

Fig. 41. Fabricated umbrella shape antenna with spiral ground plane. 
iii. Simulation and Measurement results

In order to provide design criteria for the proposed antenna, the effects of each developing geometrical shape are studied. We started with rectangular plates for both the radiator and the ground plane. The radiator dimensions are $(24 \times 12) \mathrm{mm}^{2}$ and the ground plane has the dimensions of $(40 \times 40) \mathrm{mm}^{2}$. The simulation results are shown in figure 42 . Second attempt involved converting the rectangular radiator to a semi-circular plate with radius $=12 \mathrm{~mm}$ as shown in figure 42 (same steps as in the previous sections). The electromagnetic AMC bandgap structure was used to enhance the bandwidth, increase the antenna gain and reduce the antenna size. Both the transmission line approach and reflection phase methods were used to study and redesign the performance of spiral shapes. First, the reflection coefficient and transmission coefficients $S_{11}$ and $S_{21}$ were calculated as shown in figure 43 for the two types of spiral. Figure 43 indicates that the performance of the four arms spiral ground plane is better than one arm spiral. Moreover, a large cross polarization was observed in many frequency bands in the one arm spiral one performance. This cross polarization resulted from the asymmetric geometry of the one arm spiral. Second, the reflection angle was calculated as shown in figure 44 for two types of spiral. Figure 44 indicates that the performance of spiral with four arms ground plane gives larger bandwidth and near from zero reflection phase.

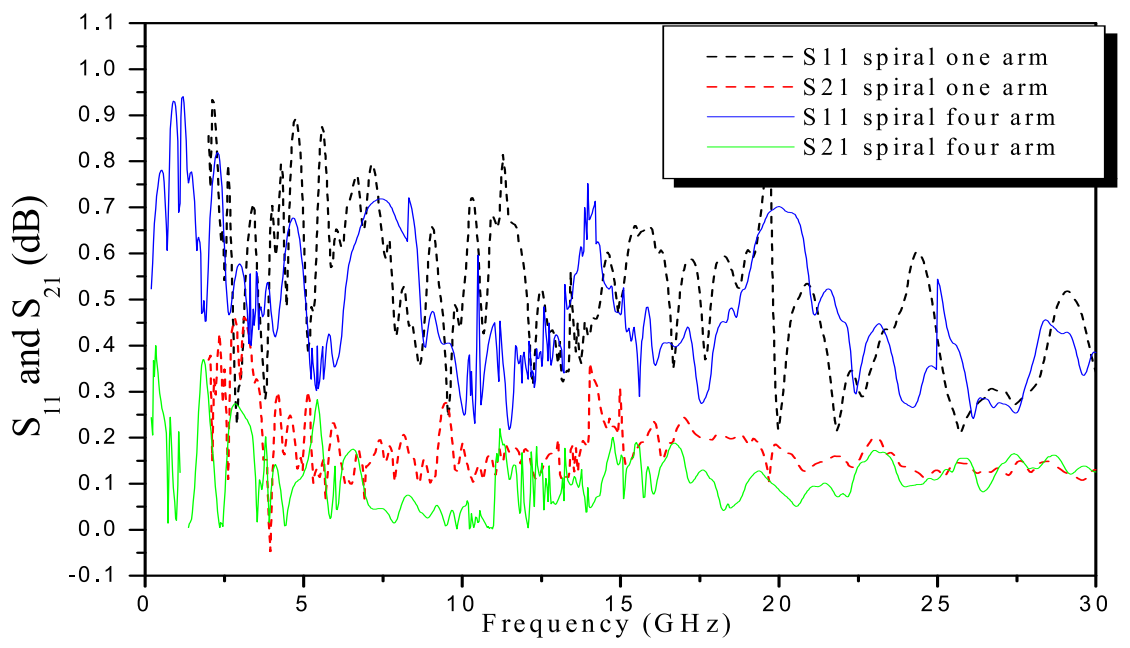

Fig. 42. Simulated results of $S_{11}$ and $S_{21}$ of spiral ground plane with one and four arms.

Then each of the two types of spirals was integrated as an antenna ground plane. The comparison between simulated and measured reflection coefficient for the antenna integrated with SAMC ground plane with one arm is shown in figure 45 . A large frequency discontinuity was also observed in this case in the frequency band from $1 \mathrm{GHz}$ up to 40 GHz. Although, the results as shown in figure 46 seem to meet the desired requirement in 
terms of surface compactness, the resulting high cross polarization makes it unacceptable in many applications. Due to the significant cross polarization, the single arm spiral geometry was not a good candidate for applications that require low cross polarization. Therefore, an alternative design that consists of four arms spiral is used [91].

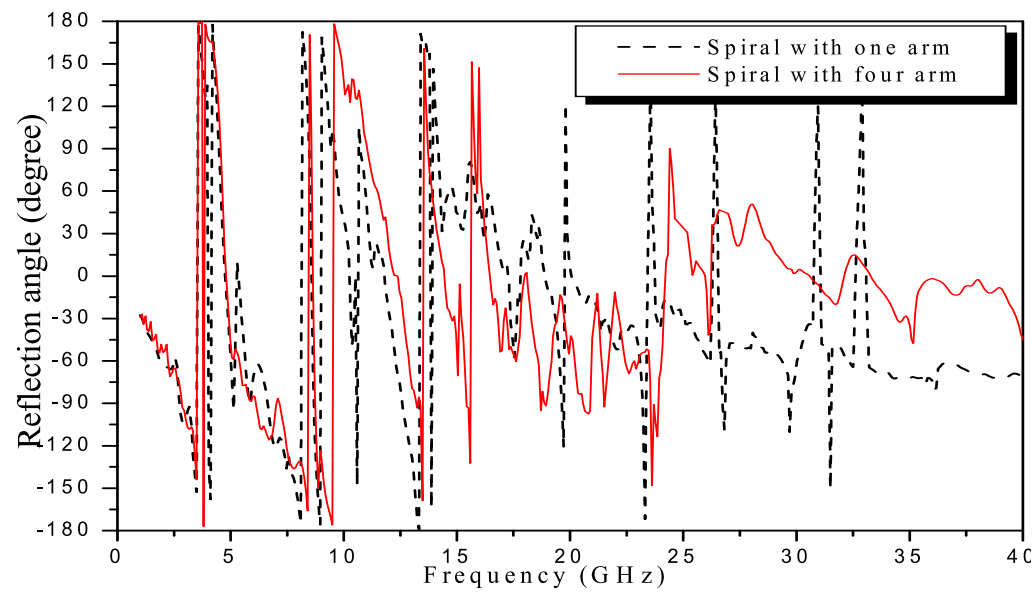

Fig. 43. The $S_{11}$ phase of spiral AMC with one and four arms.

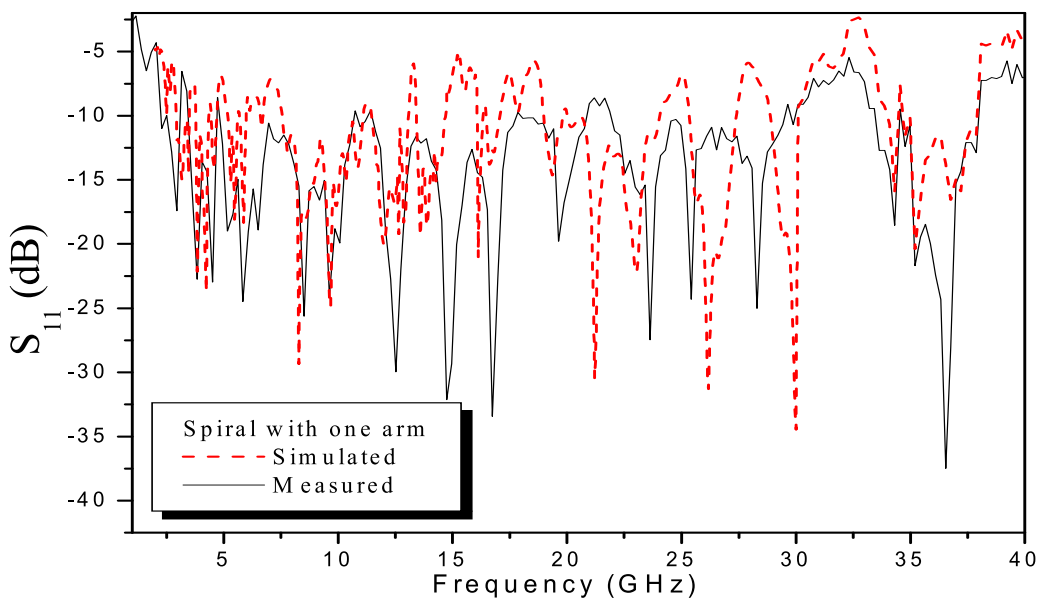

Fig. 44. Comparison between simulated and measured $S_{11}$ of proposed antenna with one arm spiral ground plane.

The four arms spiral is then applied on an antenna ground plane. Four spiral branches, each with a $1 \mathrm{~mm}=0.011 \lambda_{3.3 \mathrm{GHz}}$ width, split from the center and rotate in clockwise direction. The antenna reflection coefficient is shown in figure 46. Simulation results show that there is small discontinuity in operated antenna bandwidth between frequencies from to $0.1 \mathrm{GHz}$ to 
$1 \mathrm{GHz}$, from $5.5 \mathrm{GHz}$ to $6.5 \mathrm{GHz}$, from $7 \mathrm{GHz}$ to $7.5 \mathrm{GHz}, 13.5 \mathrm{GHz}$ to $14.5 \mathrm{GHz}$ In this case, the resonant frequency decreases as the number of spiral arms increases. The first resonant frequency is $49.45 \%$ lower than the reference geometry. This significant reduction in size for a single element leads to an attractive design feature for many wireless communication applications. Finally, both electromagnetic band-gap (EBG) drilled holes and artificial magnetic conductor (AMC) were merged to optimize the antenna performance as is shown in figure 42(c).

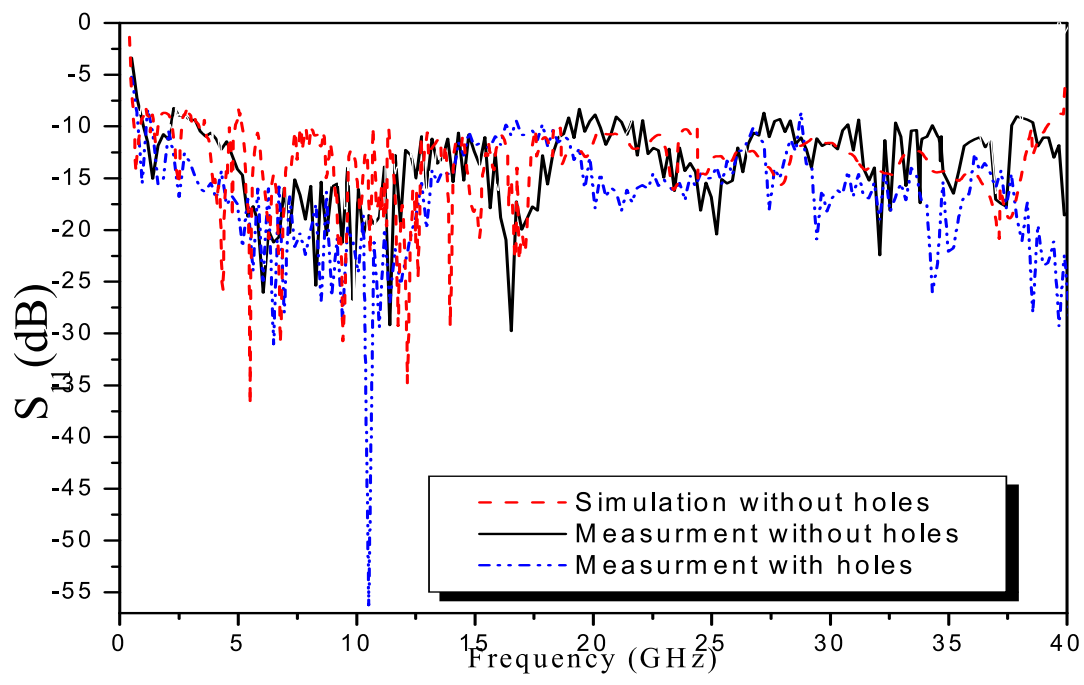

Fig. 45. The comparison between simulated and measured $S_{11}$ of the proposed antenna with four arms spiral ground plane.

\subsection{Design MMPA by using printed unequal arms V-shaped slot}

\section{i. Design Considerations}

The printed monopole antennas give very large impedance bandwidth with reasonably good radiation pattern in azimuth plane, which can be explained in two ways. To estimate the lower band-edge frequency of printed monopole antennas, the standard formulation given for monopole antenna can be used with suitable modification. The equation was worked out for the planar monopole antennas. If $h$ is the height of substrate in $\mathrm{cm}$, which is taken the same as that of an equivalent monopole and $\mathrm{L}_{\mathrm{L}}$ in $\mathrm{cm}$ is the highest effective length of the V- unequal arms monopole antenna and $\mathrm{L}_{\mathrm{f}}$ is the length of $50 \Omega$ feed line in $\mathrm{cm}$, then the lower band-edge frequency is given as shown in figure 47:

$$
\mathrm{f}_{\mathrm{L}}=\mathrm{c} / \lambda=\frac{7.2}{\left(\mathrm{~h}+\mathrm{L}_{\mathrm{L}}+\mathrm{L}_{\mathrm{f}}\right) \sqrt{\varepsilon_{\mathrm{r}}}} \mathrm{GHz}
$$

Where all dimensions $h, L_{L}$ and $L_{f}$ in $\mathrm{cm}$. 


\section{ii. Antenna Design Methodology and Geometry}

A V-shaped slot monopole with two unequal arms is proposed to achieve compact size and ultra wideband design. For the lower band $(0.75 \mathrm{GHz})$, two different-length arms of a Vshaped patch were used to excite two closely staggered resonant modes [84-86] as shown in figure 47. The two patches (triangular shaped patch and the electromagnetically coupled $\mathrm{V}$ shape are excited in the $\mathrm{TM}_{01}$ mode. The design achieves ultra bandwidth in lower band with antenna thickness less than $0.01 \lambda_{\mathrm{o}}$. Multi frequency operation is achieved by etching $\mathrm{V}$ slot with different spacing between feeding plate and triangular patch. It is placed on one side of dielectric substrate RT/D 6010 with relative permittivity 10.2 and $\tan \delta=0.002$. The dimension for the substrate is $L_{s} \times W_{s}=50 \times 50 \mathrm{~mm}^{2}$. The lowest frequency of operation is $\mathrm{f}_{\mathrm{L}}=0.75 \mathrm{GHz}$. Monopole antenna passes by many steps to reach the final novel proposed shapes. The design of the proposed antenna started from conventional shape of microstrip monopole antenna with square substrate equal to $50 \mathrm{~mm}$ using rectangular ground plane and triangular plate for antenna radiator. The dimensions are optimized by choosing a $50 \Omega$ feeding of $\mathrm{W}_{\mathrm{f}}=2.5 \mathrm{~mm}$ and $\mathrm{L}_{\mathrm{f}}=16.5 \mathrm{~mm}$. The main objective of optimization is producing broadband antenna. The second step to improve the antenna bandwidth is modifying the ground plane to partial rectangular ground plane, the ground plane dimensions are $17.5 \times 50$ $\mathrm{mm}^{2}$. Third step is using V-shaped slot with unequal arms for radiator plate as shown in figure 47. Fourth step is modifying the ground plane and optimization is used to enhance the antenna bandwidth and reduce the electrical antenna size. The triangular radiator patch with base and height $W_{p}=17 \mathrm{~mm}$ and $L_{p}=15 \mathrm{~mm}$ with arms width $W_{L}=12 \mathrm{~mm}, W_{s}=4 \mathrm{~mm}$ and arms length $\mathrm{L}_{\mathrm{L}}=36 \mathrm{~mm}, \mathrm{~L}_{\mathrm{s}}=26 \mathrm{~mm}$ and air gap separation between triangular patch and $\mathrm{V}$ shaped arms $g_{L}=2 \mathrm{~mm}, g_{s}=3.5 \mathrm{~mm}$ with feeding length $17.7 \mathrm{~mm}$. The ground plane with $\mathrm{L}_{\mathrm{t}}=12 \mathrm{~mm}$ and radius $14.4 \mathrm{~mm}$.

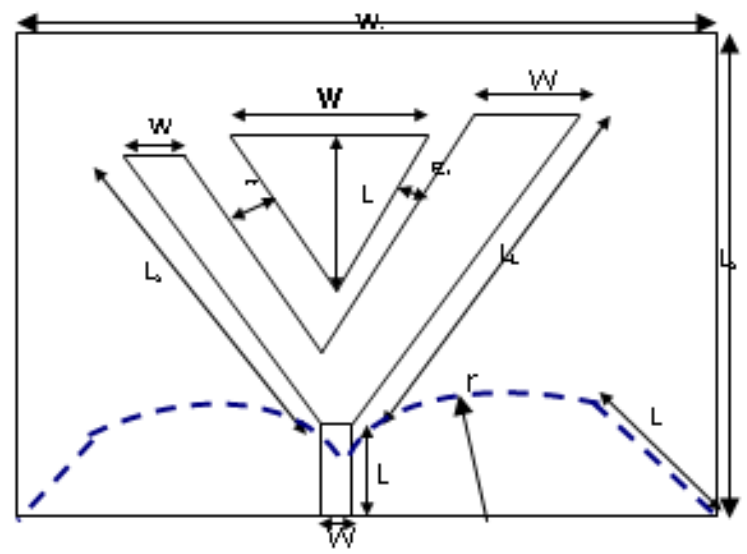

Fig. 46. The proposed antenna geometry.

$$
\begin{gathered}
2 \mathrm{a}=\lambda_{\text {guide }} \\
a / p=0.8 \sim 0.9
\end{gathered}
$$

where $a, P$ and $\lambda_{\text {guide }}$ are side length, periodicity, and guided wavelength, respectively. 
Finally, the proposed antenna is printed on metamaterial surface, first shape is using artificial magnetic conductor (AMC). As embedded spiral AMC with four arms is used to improve the impedance matching and reduce the antenna size as shown in figure 48(a) with arm width $\mathrm{g}=$ air gap $=2 \mathrm{~mm}$ at height $\mathrm{h}=1.25 \mathrm{~mm}$. Secondly, using embedded uniplanar square EBG without via and with square side length $5 \mathrm{~mm}$ for further improvement in antenna gain and bandwidth discontinuity as shown in figure 48(b) with side length $\mathrm{a}=5 \mathrm{~mm}$ and periodicity $\mathrm{P}=5.5 \mathrm{~mm}$ at height $\mathrm{h}=1.25 \mathrm{~mm}$. Finally, using electromagnetic band-gap structure (EBG) as embedded square EBG with square side length $4 \mathrm{~mm}$, periodicity $5 \mathrm{~mm}$ and via radius $=0.25 \mathrm{~mm}$ at the same previous height as shown in figure 48(c) to enhance the antenna efficiency and gain. These dimensions were selected by recomputation in a conventional way. The EBG substrate does not interfere with the near field of the antenna, and it suppresses the surface waves, which are not included in the patch antenna design [84-86]. The design of an EBG antenna has been straightforward as shown in Eqs. 5 and 6.

\section{iii. EBG Methodologies and Geometry}

The application of EBG in printed antenna design has received significant attention recently. They have mainly been used to achieve microstrip antennas on thick, high-dielectric constant substrate with optimum performance. The approach is based on using artificial substrates made of periodic metalo-dielectric resonant implants in order to have a complete forbidden band gap around the desired antenna operative frequency. Because surface waves cannot propagate along the substrate, an increased amount of radiated power couples to space waves reducing antenna losses while increasing its gain and bandwidth. In this section, a compact AMC design that minimizes the cross polarization effect of printed spiral geometry will be presented. As the number of arms or turns order increases, the equivalent inductance increases, resulting in a lower resonant frequency. Several typical printed spiral geometries are investigated in this section and their reflection phase characteristics are reported. Due to the significant cross polarization, the single and double spiral geometries are not good candidates for applications requiring low cross polarization.

A four-arm spiral is explored to eliminate the cross polarization, as shown in figure 48(a). Each arm is rotated $90^{\circ}$. Therefore, this symmetrical condition guarantees the same scattering response to the $\mathrm{x}$ - and $\mathrm{y}$-polarized incident waves. As a result, no cross polarization is observed from this structure. Second, using two dimension embedded EBG without via simplifies the fabrication process and is compatible with microwave and millimeter wave circuits. The embedded EBG surface and the AMC surface each has his own advantages. Third, a mettalo 3D-EBG with via is used: Advantages of the 3D-EBG surface is obtaining: a lower frequency and a wider bandwidth. At a given frequency, its size is smaller than the AMC design. Advantages of the uni-planar surface are obtaining a lower frequency and wider bandwidth. In addition, it is less sensitive to the incident angle and polarization.

\section{iv. Results and Discussion}

The antenna performance was investigated by both simulation and measurement as shown in figure 49. In order to provide design criteria for the proposed antenna, the effects of each developing geometrical shape are analyzed. 


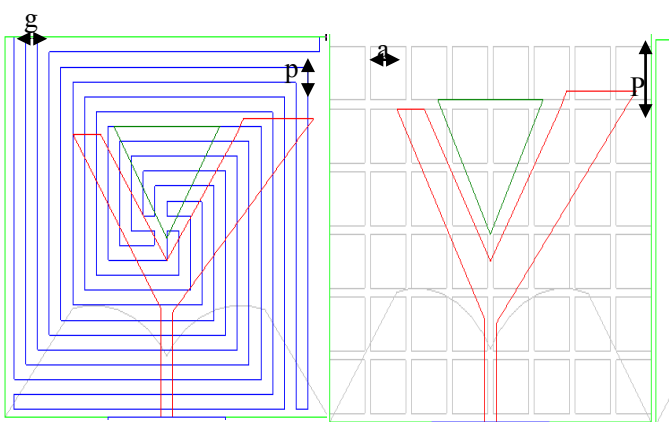

(a)

(b)

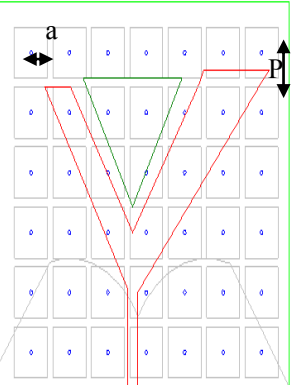

(c)

Fig. 47. The configuration of three EBG techniques.

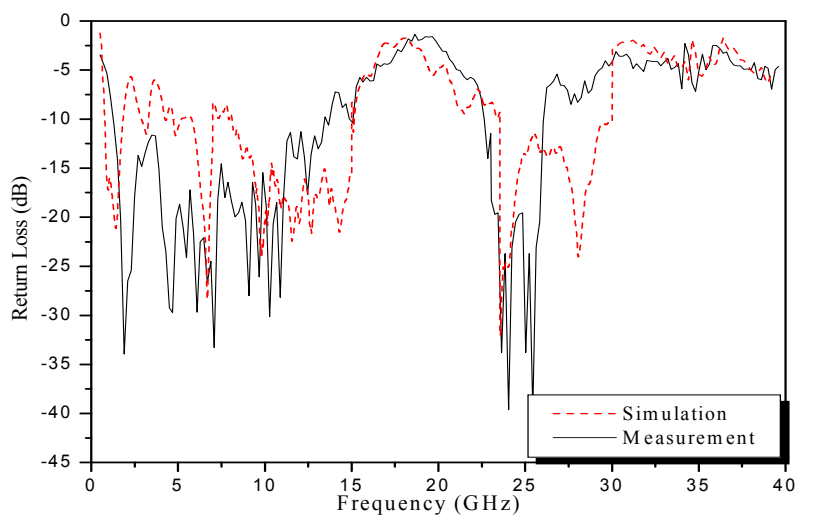

Fig. 48. Comparison between simulated and measured reflection coefficient.

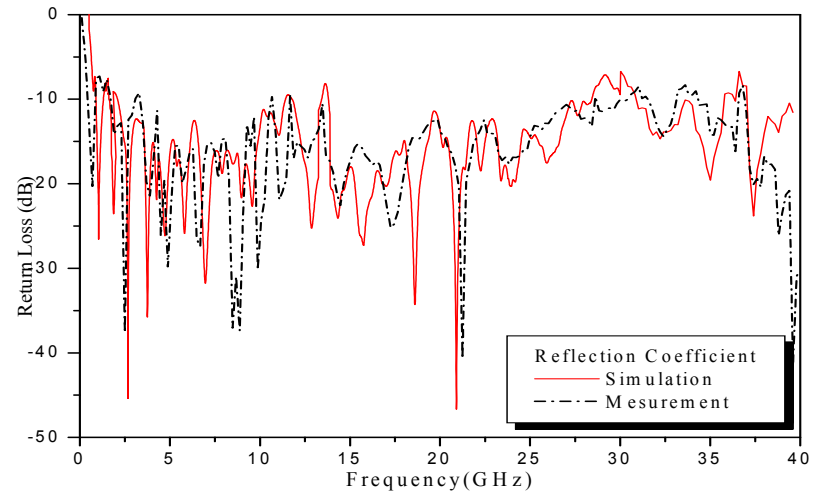

Fig. 49. Comparison between simulated and measured reflection coefficient for the proposed antenna with embedded spiral AMC 


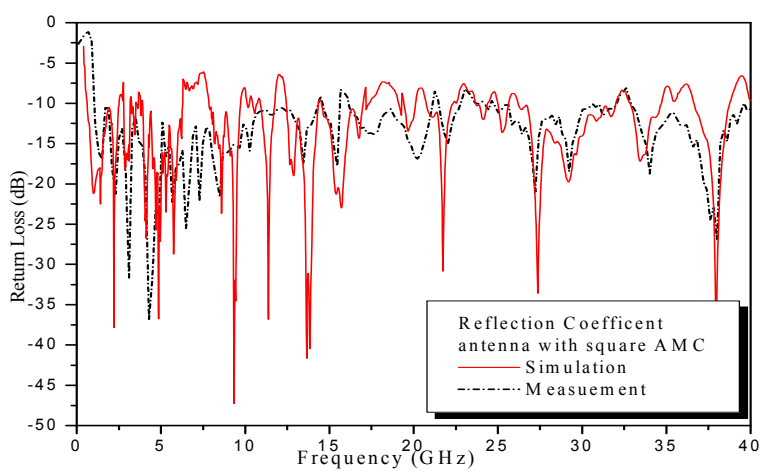

Fig. 50. Comparison between simulated and measured reflection coefficient of the proposed antenna with embedded 2D-EBG.

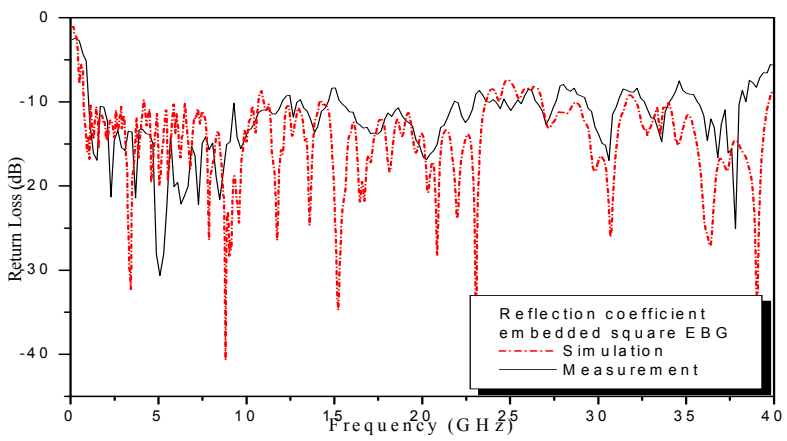

Fig. 51. Comparison between simulated and measured reflection coefficient of antenna with embedded 3D-EBG.

Metamaterials structures are used to enhance the antenna performance as bandwidth, gain and improve the reflection phase. Starting with embedded spiral AMC, the comparison between simulation and measurement of reflection coefficient is shown in figure 50, and there are bandwidth discontinuity found at frequencies from 2 to $2.5 \mathrm{GHz}, 12$ to $12.5 \mathrm{GHz}, 14$ to $14.5 \mathrm{GHz}$ and 27 to $32 \mathrm{GHz}$. However, the electrical antenna size reduced to $0.6 \mathrm{GHz}$ with reduction $65 \%$ from original size of the proposed antenna. Secondly, embedded square uniplanar 2D-EBG were used. The comparison between simulation and measurement is shown in figure 51 and the bandwidth discontinuities occur at frequencies from 5 to $8 \mathrm{GHz}$, from 12 to 13,17 to $18.5 \mathrm{GHz}$ and from 32 to $34.5 \mathrm{GHz}$. Thirdly, embedded 3D-EBG is used for further improvement in antenna gain and bandwidth. The bandwidth discontinuities occur at frequencies from 11 to $12 \mathrm{GHz}$ and from 26 to $26.5 \mathrm{GHz}$ as shown in figure 52 . It may be noted that not only improvement in the bandwidth is achieved but also the antenna size was reduced to about $65 \%$ from that of the proposed monopole antenna without EBG. From these figures, one can notice that there are small discrepancies between the simulated and measured results. This may be attributed due to the same reasons stated before. Antenna gain for three antenna structures are shown in figure 53. This figure shows that, the antenna with embedded 3D-EBG has the antenna gain response along the operating band with average gain of about of $13 \mathrm{dBi}$ followed by $2 \mathrm{D}-\mathrm{EBG}$ with average gain $11 \mathrm{dBi}$ and spiral AMC with 9dBi. The fabricated antenna is shown in figure 54. 


\subsection{Co-planar boat MPA with modified ground plane by using EBG}

\section{i. Antenna Geometry}

The geometry of the proposed antenna is shown in figure 55, where an equi-lateral triangular patch with $\mathrm{L}_{\mathrm{r}}=70 \mathrm{~mm}$ is placed co-planar to a finite ground plane that has a trapezoidal shape with size of $\mathbf{W}_{\mathrm{g} 1}=30 \mathrm{~mm}, \mathbf{W}_{\mathrm{g} 2}=63 \mathrm{~mm}$, hieght $\mathbf{L}_{\mathrm{g}}=21 \mathrm{~mm}$. The dielectric substrate used is FR4 with dielectric constant $\varepsilon_{\mathrm{r}}=4.7$ and dimension $100 \times 100 \mathrm{~mm}^{2}$ with thickness $\mathbf{h}=3.2 \mathrm{~mm}$. The patch is proximity fed by a $50 \Omega$ microstrip line at the fundamental frequency $3.3 \mathrm{GHz}$ with line length and width of $\mathbf{L}_{\mathrm{f}}=63 \mathrm{~mm}$ and $\mathbf{W}_{\mathrm{f}}=5.5 \mathrm{~mm}$, respectively. The top and side views of the proposed antenna are shown in figure 55. To obtain a good impedance matching, the end of the feed line has to extend beyond the centre of the patch. Initially, different dimensions of the MPA were used in order to minimize the size of the patch antenna with maximizing the bandwidth at the same time. The antenna geometry of the whole structure should be optimized (the ground plane dimension, separation between the patch and the ground and feed line position) to obtain the best possible impedance bandwidth [87].

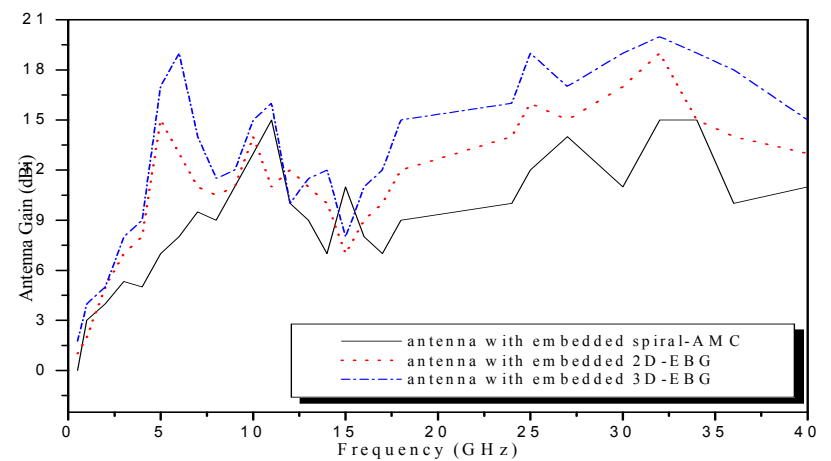

Fig. 52. Comparison of gain for the studied EBG structures.

Second part of this section, is using 2D-EBG etched in the feeding line to improve impedance matching with head square dimension $a=2 \mathrm{~mm}$, slot length $\mathbf{L}_{\mathbf{d}}=1.25 \mathrm{~mm}$ with width g=0.5mm and periodicity $\mathbf{P}=4 \mathrm{~mm}$. An etched 2D-EBG in $50 \Omega$ feed line disturbs the shield current distribution in the feed line. This disturbance can change the characteristics of the transmission line since it increases the effective capacitance and inductance of a transmission line, respectively.

Finally, using the four arms spiral AMC to reduce the antenna size by adding inductance component. A larger equivalent inductance may be realized with a larger number of spiral turns. However, as revealed in this chapter, if the unit geometry is not symmetric with respect to the polarizations of the incident waves, the AMC surface generates a high level of cross polarization. Thus, the behavior of reflection phase may not be applicable in the designated frequency band of operation. Using four arms spiral shape, and each arm rotates $90^{\circ}$ can exactly recover itself. Therefore, this symmetrical condition guarantees the same scattering response to the $x$ - and $y$-polarized incident waves. As a result, no cross polarization was observed from this structure. Therefore, with this design the compactness of geometry is achieved without generating the cross polarization level. The dimension of the spiral arm width $\boldsymbol{W}_{\mathbf{d}}$ is equal to separation between arms $=5 \mathrm{~mm}\left(0.05 \lambda_{\mathrm{g}}\right)$ and the largest spiral length $\mathbf{L}_{\mathrm{s}}=80 \mathrm{~mm}$ as shown in figure $55(\mathrm{~b})$. 


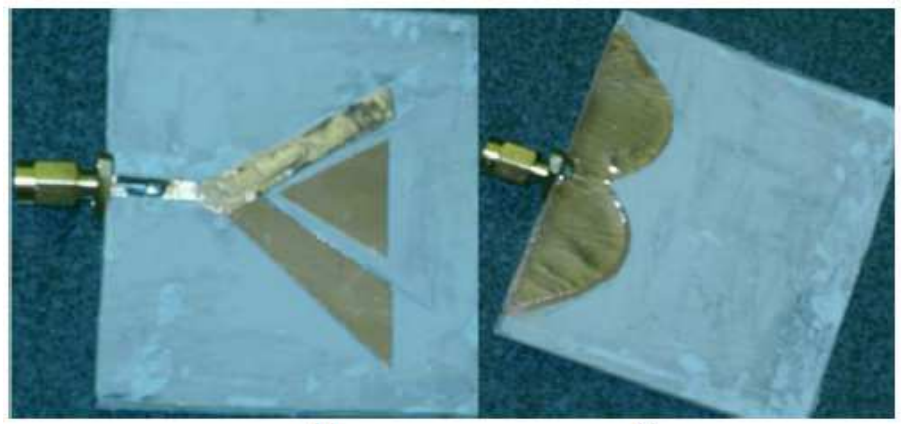

(a)

(b)

Fig. 53. The fabricated antenna (a) radiator and (b) ground plane

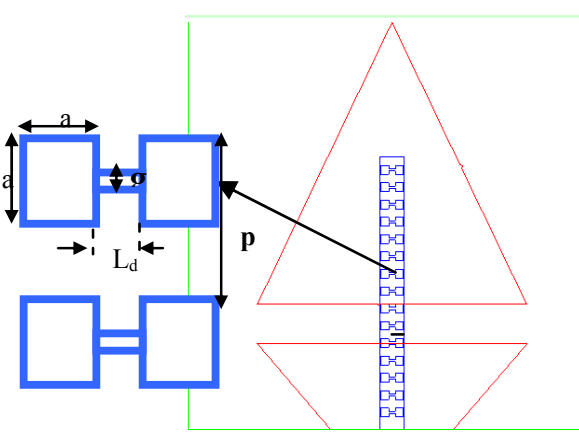

(a)

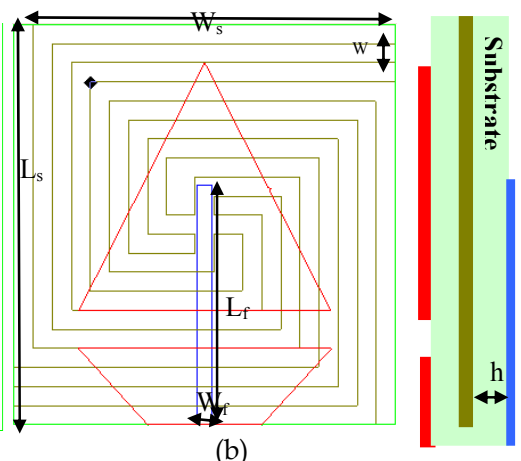

(b)

Fig. 54. Top and side view of the proposed antenna., (a) the proposed antenna with EBG elevation and $(\mathrm{bc})$ side view.

\section{ii. Simulated and Measured Results}

The antenna performance was investigated by using simulations and verified experimentally by fabricating the antenna using photolithographic techniques. In order to provide design criteria for the proposed antenna, the effects of each developing geometrical dimensions were analyzed. The effect of feeding line length was studied. There is an optimum value of the feed length which is $63 \mathrm{~mm}$. The simulated return loss with various ground plane width, $\mathbf{W}_{\mathbf{g} 1}$ studied and it is found that larger width gives a broader bandwidth as well as a lower return loss magnitude. The proposed antenna is sensitive to $\mathbf{L}_{\mathrm{g}}$ and in fact broadband performance is obtained for $\mathbf{L}_{\mathrm{g}}=21 \mathrm{~mm}$. It is known that in proximity fed patch antennas the position of the feed line under patch is important. The simulated return loss with various separations between the triangular radiator and the trapezoidal ground $\mathbf{W}_{\text {gap }}$ plane is also studied as shown in figure 56 where it can be seen that larger gap width gives a lower bandwidth as well as a lower return loss magnitude. However, there is an optimum separation $\mathbf{W}_{\text {gap }}$ which gives good reflection coefficient and bandwidth as shown in figure 57. Figure 58 shows a comparison between simulated and measured reflection coefficient of the proposed antenna with optimum dimensions. Figure 58 shows comparison between simulated and measured reflection coefficient. There is an improvement in bandwidth from $1 \mathrm{GHz}$ to about $40 \mathrm{GHz}$ with 
discontinuity band in the operating region. An embedded four arms spiral AMC was added at height $\mathrm{h}=1.6 \mathrm{~mm}$ from the ground plane to decrease the bandwidth discontinuity and cross polarization as well as increasing the antenna gain.

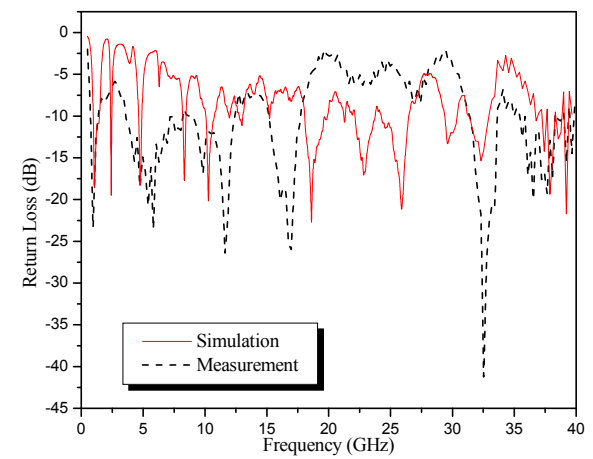

Fig. 55. Comparison between simulated and measured reflection coefficient of the antenna without EBG

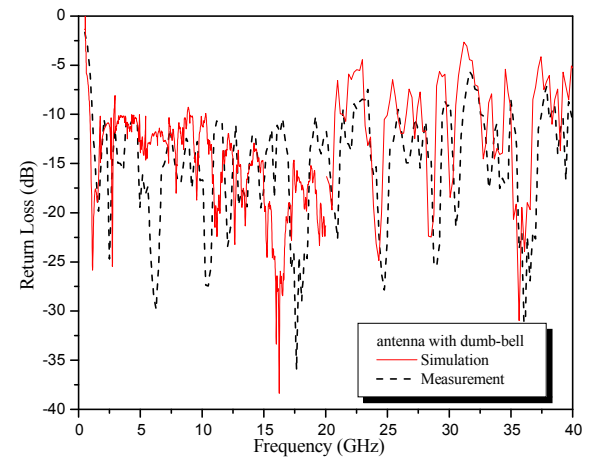

Fig. 56. Comparison between simulated and measured return loss for the antenna with dumb-bell

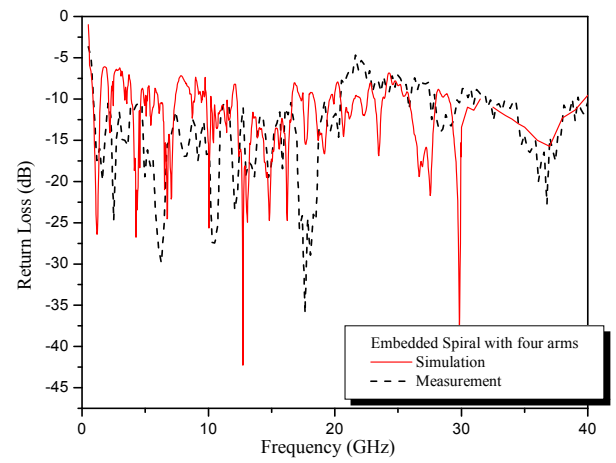

Fig. 57. Comparison between simulated and measured return loss for the proposed antenna with embedded SAMC under the antenna radiator. 


\section{Design of microstrip patch antenna arrays with EBG}

After discussing EBG applications in single microstrip patch antennas, we now present how EBG can help to improve the performance of MPAA. The mutual coupling between array elements as an important parameter in array design. Strong mutual coupling could reduce the array efficiency and cause the scan blindness in phased array systems. [93] Therefore, the electromagnetic band gap (EBG) structures are used to reduce the coupling between array elements.

There are diverse forms of EBG structures [97], and novel designs such as EBG structures integrated with active device and multilayer EBG structures have been proposed recently. This section focuses on different types of EBG structures. Its band-gap features are revealed for two reasons, suppression of surface-wave propagation, and in-phase reflection coefficient. The feature of surface-wave suppression helps to improve antenna's performance such as increasing the antenna gain and reducing back radiation [92]. Meanwhile, the in-phase reflection feature leads to low profile antenna designs [94]. This section concentrates on the surface-wave suppression effect of the EBG structure and its application to reduce the mutual coupling of MPAA. To explore the surface-wave suppression effect, the propagating fields of an infinitesimal dipole source with and without the EBG structure are simulated and a frequency stop-band for the field propagation is identified. Furthermore, the propagating near fields at frequencies inside and outside the band gap is graphically presented for a clear understanding of the physics of the EBG structure. It is worthwhile to point out that this band-gap study is closely associated with specific antenna applications such as MPAA. Applications of MPAA on high dielectric constant substrates are of special interest due to their compact size and conformability with the monolithic microwave integrated circuit (MMIC). However, the utilization of a high dielectric constant substrate has some drawbacks. Among these are narrower bandwidths and pronounced surface waves. The bandwidth can be recovered using a thick substrate, yet this excites severe surface waves. The generation of surface waves decreases the antenna efficiency and degrades the antenna pattern. Furthermore, it increases the mutual coupling of the antenna array which causes the blind angle of a scanning array. Several methods have been proposed to reduce the effects of surface waves. One suggested approach is the synthesized substrate that lowers the effective dielectric constant of the substrate either under or around the patch. Another approach is to use a reduced surface wave patch antenna. The EBG structures are also used to improve the antenna performance. However, most researchers only study the EBG effects on a MPA element, and to the best of our knowledge there are no comprehensive results reported for antenna arrays. The mutual coupling of MPAA is parametrically investigated, including the E- and H-coupling directions, different substrate thickness, and various dielectric constants. In both coupling directions, increasing the substrate thickness will increase the mutual coupling. However, the effect of the dielectric constant on mutual coupling is different at various coupling directions. It is found that for the Eplane coupling is stronger on a high permittivity substrate than that on a low permittivity substrate. In contrast, for the H-plane coupled cases the mutual coupling is weaker on a high permittivity substrate than that on a low permittivity substrate. This difference is due to surface waves propagating along the E-plane direction. To reduce the strong mutual coupling of the E-plane coupled MPAA on a thick and high permittivity substrate, 
the EBG structure is inserted between antenna elements. When the EBG parameters are properly designed, the pronounced surface waves are suppressed, resulting in a low mutual coupling. This method is compared with previous methods such as cavity backed patch antennas. The EBG structure exhibits a better capability in lowering the mutual coupling than other approaches.

\subsection{Different types of EBG for mutual coupling reduction}

Utilization of electromagnetic band-gap (EBG) structures is becoming attractive in the electromagnetic and antenna community. In this chapter we describe three ways to improve the performance of microstrip antenna arrays by using 3D-EBG, 2D-EBG and defected ground structure DGS. At the end of this chapter as conclusion, we make comparison between the effects of these methods on the array characteristics.

\subsection{Mutual coupling reduction by using the 2D-EBG structure}

Surface waves are undesired because when a patch antenna radiates, a portion of total available radiated power becomes trapped along the surface of the substrate. It can extract total available power for radiation to space wave as well as there is harmonic frequency created [96]. For arrays, surface waves have a significant impact on the mutual coupling between array elements. One solution to reduce surface waves is using electromagnetic band-gap (EBG) or photonic band-gap structure (PBG). Many shapes of EBG slot have been studied for single element microstrip antenna such as circles, dumb-bells and squares. However, not many have realized in antenna arrays. It has been demonstrated that the EBG structure will lead to a reduction in the side-lobe levels and improvements in the front to back ratio and overall antenna efficiency for the radiation pattern. However, the antenna in the above mentioned references has only one patch. The unique capability of the EBG structure to reduce the mutual coupling between elements of an antenna array was demonstrated. The side lobe of the antenna with one patch is due to surface-wave diffraction at the edges of the antenna substrate. For antenna array, the side lobe is related to the pattern of the individual antenna, location of antenna in the array and the relative amplitudes of excitation. In addition, the mutual coupling between radiators affects the current distribution on the antenna and resulted in increased side lobes.

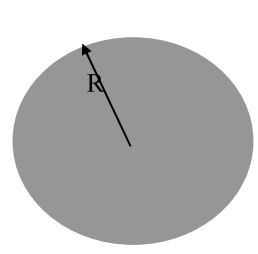

(a)

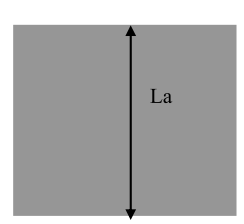

(b)

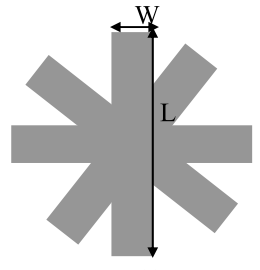

(c)

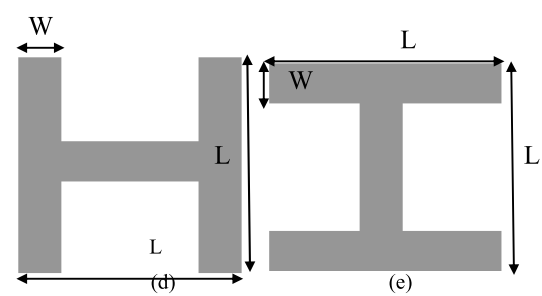

(e)

Fig. 58. The different shapes of one unit cell of 2D-EBG: (a) conventional circle, (b) conventional square, (c) star, (d) H-shape and (e) I-shape. 
In this section, MPA array with three different shapes of 2D-EBG as star, $\mathrm{H}$ shape and Ishape slot etched on the ground plane are designed, simulated and measured. In this study, harmonic suppression and reduction of the mutual coupling effect are investigated by proposing these new shapes of 2D-EBG. The obtained results demonstrate that the 2D-EBG not only reduces the mutual coupling between the patches of antenna array, but also suppresses the second harmonic, reduces the side lobe level and gives results better than conventional 2D-EBG shapes as circle and square. It is also shown that the novel shapes of 2D-EBG on the ground plane increases the gain of the antenna array.

\section{i. Configurations of 2D-EBG Shapes}

Three different shapes of 2D-EBG are presented, as shown in figure 59; the three shapes are compared with familiar conventional shapes as circular and square shapes 2D-EBG by using transmission line approach. The proposed EBG units are composed of several rectangularshape slots (of length $L$ and width $W$ ). This EBG cell can provide a cutoff frequency and attenuation pole. It is well known that an attenuation pole can be generated by a combination of the inductance and capacitance elements, which presents circuit model for the cell for all 2D-EBG structures. Here, the capacitance is provided by the transverse slot and the inductance by different shapes slots. For star shape there are four rectangular slots with $L=5 \mathrm{~mm}, W=1 \mathrm{~mm}$ at angles $=00^{\circ}, 45^{\circ}, 90^{\circ}$ and $135^{\circ}$. The second shape, which is the $\mathrm{H}$ shaped slot, consists of three rectangular slots with the same previous dimensions. The third shape is the I-shaped slot, which is obtained by rotating H-shape by 90 . The substrate with a dielectric constant of 10.2, loss tangent of 0.0019 and thickness of $2.5 \mathrm{~mm}$ is considered here. The microstrip feeding line on top plane has a width $W_{f}=2.3 \mathrm{~mm}$, corresponding to $50 \Omega$ characteristic impedance. 2D-EBG cells are etched on the ground plane with periodicity $\boldsymbol{P}=7 \mathrm{~mm}$ and ratio $\mathrm{L} / \mathrm{p} \cong 0.7$. Then the reflection and transmission coefficients $\left(\mathrm{S}_{11}\right.$ and $\left.\mathrm{S}_{21}\right)$ are calculated using the high frequency structure simulator (HFSS).

\section{ii. Antenna Array Design}

Consider an ordinary antenna array with two elements, at $5.2 \mathrm{GHz}$, the dimensions of the patches are patch width $W_{p}=8 \mathrm{~mm}$, patch length $L_{p}=7.5 \mathrm{~mm}$ and microstrip feed line with length $L_{f}, L_{s}=20 \mathrm{~mm}, 13 \mathrm{~mm}$, respectively, and the distance between the patches is $L_{B}=19$ $\mathrm{mm}\left(0.44 \lambda_{5.2 \mathrm{GHz}}\right)$. It can be seen that the antenna will radiate energy at a harmonic frequency of $7.5 \mathrm{GHz}$. In order to suppress such harmonics, the band-stop characteristic of the EBG structure may be used. In this section, which is simply 2D-EBG cells are etched on the ground metal sheet. The two separate array elements are also studied to measure the mutual coupling between the two patches in MIMO arrays.

\section{iii. Results and Discussion}

The response that is shown in figure 60 presents both conventional shapes as circular and square 2D-EBG as well as the three new shapes. The second step, is applying these shapes to the ground plane of two element array antenna. The results indicate that the harmonic at 7.5 $\mathrm{GHz}$ is indeed suppressed as well as reducing array antenna size by about $7.5 \%$ for $\mathrm{H}$ shape, $8 \%$ for star-shape and for I-shape reduction in size reaches $15 \%$. According to the characteristics of EBG, the surface wave can also be suppressed. 

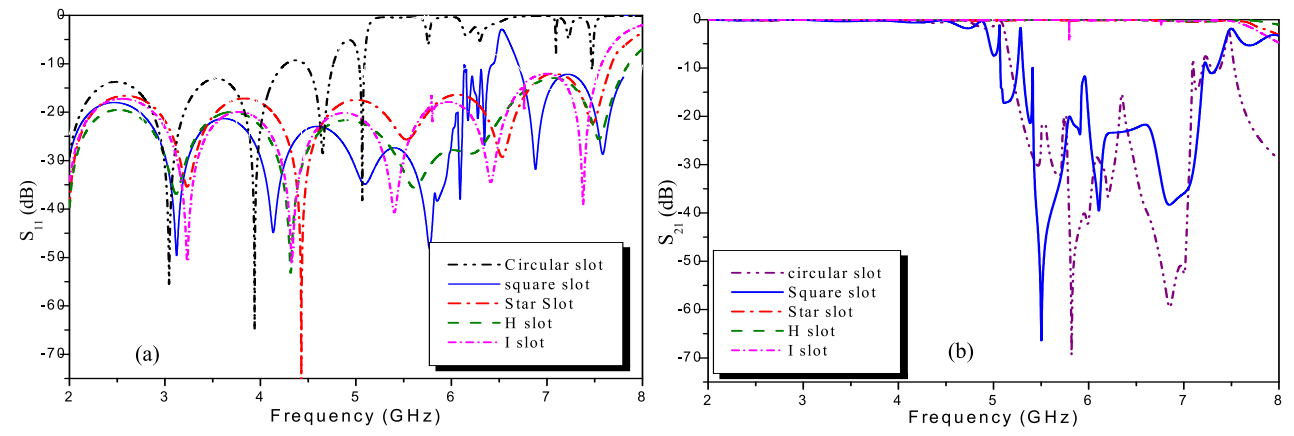

Fig. 59. (a) The reflection and (b) transmission coefficients for different 2D-EBG shapes.

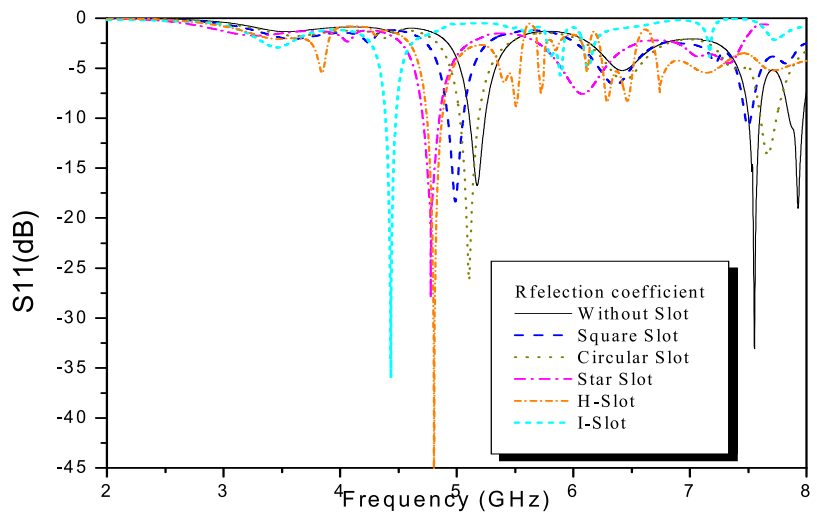

Fig. 60. The reflection coefficient for different MPAA with 2D -EBG.

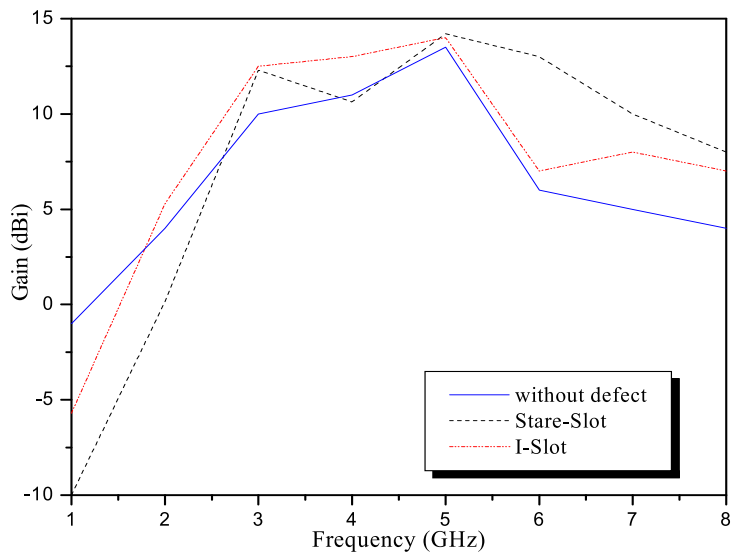

Fig. 61. The Gain response for different shapes of MPAA without and with 2D-EBG shapes. 


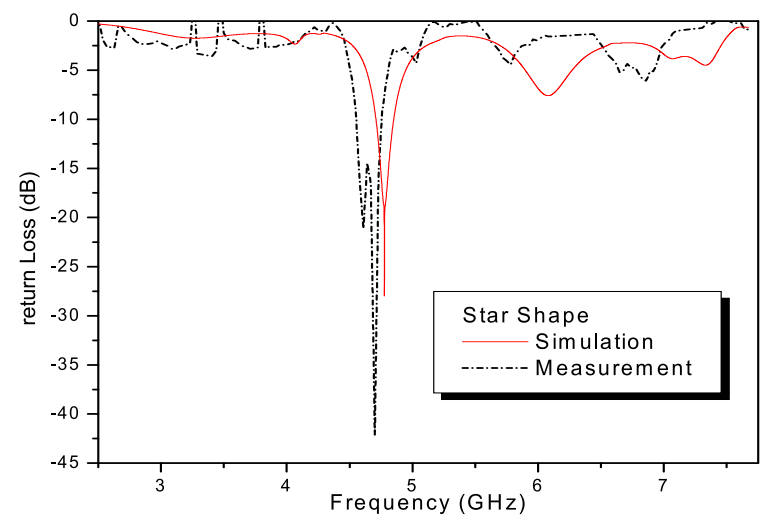

Fig. 62. Comparison between measured and simulated reflection coefficients for star shaped slot 2D-EBG.

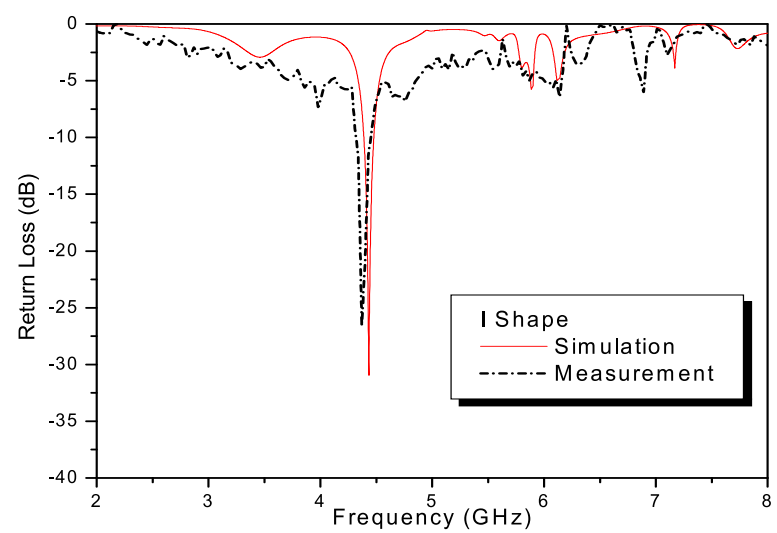

Fig. 63. Comparison between measured and simulated reflection coefficients for I shaped slot 2D-EBG.

For effective suppression of the harmonics, and for effective suppression of the surface waves, a periodic structure surrounding the patches, in addition to underneath the patches, are necessary [98]. The array performance of the conventional and the three new shapes of 2D-EBG are given in table 7. From table 7 and figure 61, one can notice that; I shape gives maximum reduction in resonant array frequency than other shapes so reduce the electrical array size, star shape gives maximum average antenna gain and minimum mutual coupling while $\mathrm{H}$ shape gives larger antenna bandwidth than others. By using conventional shapes as circular and square EBG, the concentration of surface current decreases but not eliminated. However, the gain of the two-element array antenna is also studied for different array antennas with and without 2D-EBG as shown in figure 62. The comparisons between measured and simulated reflection coefficients are shown in figures 63 and 64 for the star and I shape, respectively. From table 7 it notes that, at higher frequencies the antenna gain with 2D-EBG is better than that without EBG by about $9 \mathrm{dBi}$ maximum difference and $3 \mathrm{dBi}$ in average over the entire antenna band which verifies the harmonic suppression behavior. 
In addition the average efficiency of the array is also studied over the operating band. The average array efficiency with conventional 2D-EBG is lower than that without by about $15 \%$ while with three new shapes of 2D-EBG is lower by about $10 \%$.

\begin{tabular}{|c|c|c|c|c|c|c|c|}
\hline $\begin{array}{l}\text { Shape of } \\
\text { 2D-EBG }\end{array}$ & $\begin{array}{c}\text { Antenna } \\
\text { Gain dB } \\
@ 5.2 \mathrm{GHz}\end{array}$ & $\begin{array}{c}\text { Mutual } \\
\text { Coupling } \\
\left|S_{21}\right|\end{array}$ & $\begin{array}{c}\text { Harmonic } \\
\text { level dB }\end{array}$ & \begin{tabular}{|l|} 
Reflection \\
Coefficient
\end{tabular} & $\begin{array}{c}\mathrm{F}_{\mathbf{o}} \\
\mathrm{GHz}\end{array}$ & $\begin{array}{c}\text { BW } \\
\%\end{array}$ & $\begin{array}{c}\text { Defect } \\
\text { Geometry, } \\
\text { Periodicity } \\
\text { X direction /Y } \\
\text { direction }\end{array}$ \\
\hline Without & 10 & $-16 \mathrm{~dB}$ & -35 & $-17 \mathrm{~dB}$ & 5.1 & 3 & \\
\hline Square & 13.5 & $-18.5 \mathrm{~dB}$ & -10 & $-17.5 \mathrm{~dB}$ & 5 & 4 & $\begin{array}{l}\text { Side dimension } \\
4 \mathrm{~mm}, 6 \mathrm{~mm}, 6 \mathrm{~mm}\end{array}$ \\
\hline Circular & 13 & $-19 \mathrm{~dB}$ & -15 & $-20 \mathrm{~dB}$ & 4.8 & 5 & $\begin{array}{c}\text { Radius } 2 \mathrm{~mm}, \\
2 \mathrm{~mm}, 2 \mathrm{~mm}\end{array}$ \\
\hline Star & 13.75 & $-40 \mathrm{~dB}$ & -9 & $-30 \mathrm{~dB}$ & 4.75 & 5 & $\begin{array}{l}\text { Side length } 1 \mathrm{x} \\
4 \mathrm{~mm}^{2}, 6 \mathrm{~mm}, \\
6 \mathrm{~mm}\end{array}$ \\
\hline $\mathrm{H}$ & 12.75 & $-20 \mathrm{~dB}$ & -10 & $-40 \mathrm{~dB}$ & 5 & 5.1 & $\begin{array}{c}\text { Side length } \\
1 \times 5 \mathrm{~mm}^{2}, 6 \mathrm{~mm} \text {, } \\
6 \mathrm{~mm}\end{array}$ \\
\hline I & 11 & $-30 \mathrm{~dB}$ & -7 & $-45 \mathrm{~dB}$ & 4 & 5 & $\begin{array}{c}\text { Side length } \\
1 \times 5 \mathrm{~mm}^{2}, 6 \mathrm{~mm}, \\
6 \mathrm{~mm}\end{array}$ \\
\hline
\end{tabular}

Table 7. The effect of different 2D-EBG shapes on the antenna performance.

\subsection{Novel shapes of low mutual coupling 2X2 MPAA by using DGS}

In this section, we propose new shapes of DGS structures integrated with microstrip array elements that suppress surface wave and lead to a high isolation between array elements, hence reduce the mutual coupling. Many published papers have made use of relatively complex periodic electromagnetic band-gap (EBG) structures to reduce the mutual coupling. In this section we propose improvement in $\mathrm{E}$ and $\mathrm{H}$ plane coupling by about $4 \mathrm{~dB}$ than dumb-bell shapes presented in [95].

i. DGS Configurations and Response

Figure 65 displays the different shapes of the DGS etched on the metallic ground plane. The different cell shapes of DGS are dumb-bell, H, E, $\mathrm{H}$ with inverted $\mathrm{H}$ and back-to-back $\mathrm{E}$ as shown in figure 65 (a)-(e), respectively.

The responses of these shapes are compared in figure 66 by using the conventional $50 \Omega$ microstrip transmission line approach. The transmission and reflection coefficients of the conventional dumbbell and new shapes of DGS are presented in figure 66(a) and 66(b), respectively. It is demonstrated that when an aperture etched on the ground plane, the effective inductance of the microstrip increases and the width of the connecting gap determines the shunt capacitance. The inductance and capacitance level may be independently controlled by the equivalent aperture area and gap width, respectively. 


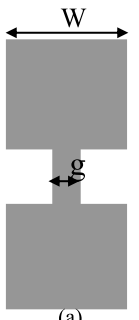

(a)

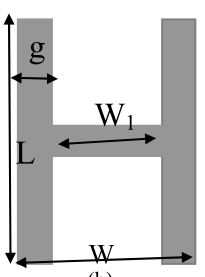

(b)

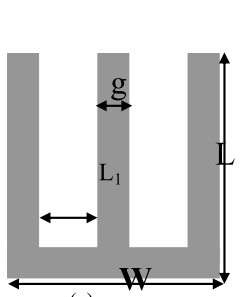

(c)

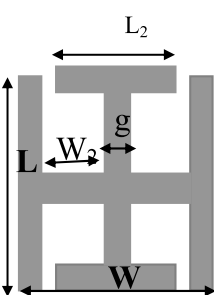

(d)

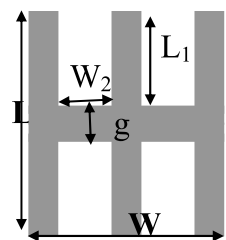

(e)

Fig. 64. Different DGS shapes (a) dumbbell, (b) H shape, (c)E, (d) H with inverted $\mathrm{H}$ and (e) back to back E shape.

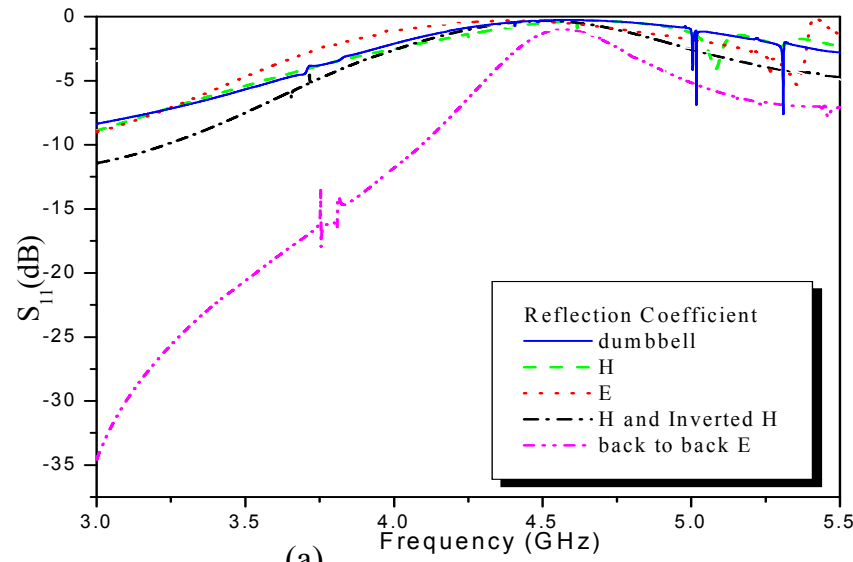

(a)

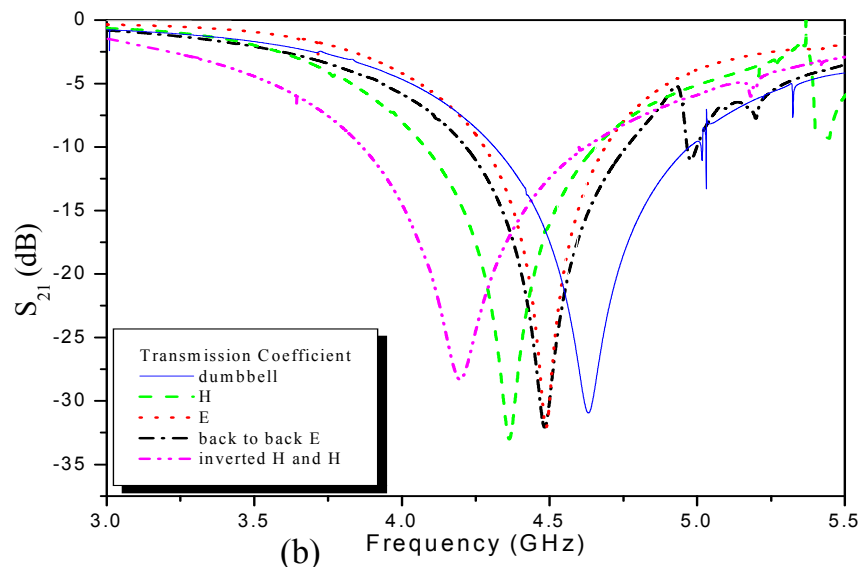

Fig. 65. (a) Reflection coefficient, and (b) Transmission coefficient for different DGS. 
On the other hand, it should be noted that etching holes in the ground plane of DGS structures degrades the isolation characteristic of the ground plane thus obtaining large backward radiation pattern. However, by using these new shapes of the DGS structures, the effective apertures area are reduced thus there are improvements on the array E- and $\mathrm{H}$ plane radiation patters. Figure 66 indicates that all new shapes $(E, H$, back-to-back $E$ and $H$ with inverted $\mathrm{H}$ ) give wider stop-band filter response than conventional dumbbell shapes, hence improve the antenna characteristics.

\subsubsection{Study of mutual coupling reduction}

Due to high excitation of surface waves in both $\mathrm{E}$ and $H$-plane coupling between microstrip array patches an investigation was carried out by simulation to study the effect of the newly DGS shapes on the mutual coupling. Two substrates with thickness of 1.6 $\mathrm{mm}$ and $2.5 \mathrm{~mm}$ and permittivity of 4.7 and 10.2, respectively, were used in the simulations. This difference is due to surface waves propagating along the E-plane direction, which can be easily viewed from the provided near field plots. For microstrip feed array as shown in figure 67 (a) the array element is with dimensions $\boldsymbol{W}_{p} \times \boldsymbol{L}_{p}=16 \mathrm{~mm}$ $x 12 \mathrm{~mm}$ and $L_{a}=15 \mathrm{~mm}$. While the edge-to-edge separation is $L_{B}>\lambda_{0} / 2=30 \mathrm{~mm}$ at a designed resonant frequency $5.25 \mathrm{GHz}$ and printed on FR4 substrate with dielectric constant 4.7, height $1.6 \mathrm{~mm}$ and substrate dimensions $\boldsymbol{W}_{s} \times \boldsymbol{L}_{s}=53 \mathrm{~mm} \times 53 \mathrm{~mm}\left(\boldsymbol{\lambda}_{0} \times \boldsymbol{\lambda}_{0}\right)$. Figure $67(\mathrm{~b})$ illustrates the layout of a 4-element coaxial feed microstrip array on the defected ground plane with substrate dimensions $63 \mathrm{~mm} \times 63 \mathrm{~mm}=\left(\lambda_{0} \times \lambda_{0}\right)$ at a designed frequency of 4.75 GHz. The array patch is with dimensions $W p \times L p=10.5 \mathrm{~mm} \times 8.5 \mathrm{~mm}$ with edge to edge separation distance $d>\lambda_{0} / 2=35 \mathrm{~mm}$ and printed on RT/D6010 substrate with dielectric constant 10.2 and height $2.5 \mathrm{~mm}$. In coaxial feed, only element 2 is excited while other elements 1, 3, 4 are $50 \Omega$ terminated. The DGS dimensions for a band-gap at the resonant frequency of the antenna are optimized shapes. It consists of rectangular strips, each with dimensions $L X g=7.5 \mathrm{~mm} \times 1.5 \mathrm{~mm}$ and width $W=7.5 \mathrm{~mm}, W=3.5 \mathrm{~mm}$ and $W=1.5 \mathrm{~mm}$ with length $L=3 \mathrm{~mm}$ and $L=3.5 \mathrm{~mm}$ for E-shape, $H$ with inverted $H$ shape and back to back $E$ shaped, respectively. Figure 68 shows the reflection coefficient of the microstrip line feed 2x2 MPAA at low dielectric constant FR4 substrate without and with different shapes of DGS [92]. It is observed that DGS antenna resonant frequency shifts towards lower values with respect to the conventional antenna. This small frequency shift is due to wave slowing effects of DGS. Both back-to-back $\mathrm{E}$ and $\mathrm{H}$ with inverted $\mathrm{H}$ give better response than other shapes. Table 8 summarizes all the MPAA characteristics without and with DGS. Figure 69 shows the comparison between simulated and measured reflection coefficient of the MPAA with back-to-back E and H with inverted H DGS. Figure 70 is a plot of all responses of the coaxial fed 2x2 MPAA at high dielectric constant RT/D6010 substrate with different shapes of DGS together with the conventional array. $S_{11}$, figure 70 (a), shows that DGS structure shifts down the antenna resonant frequency as compared to the conventional MPAA. The E-plane mutual coupling $S_{21}$ are shown in figure $70(\mathrm{~b})$. The conventional antenna shows a very strong coupling of $-9.63 \mathrm{~dB}$ due to surface waves pronounced in thick, high permittivity substrate. Since the resonant frequency $4.75 \mathrm{GHz}$ of the antenna falls inside the DGS band-gap, surface waves are suppressed and simulations show that mutual coupling drops to $-20 \mathrm{~dB}$ with $\mathrm{H}$ and inverted $\mathrm{H}$-shape that is lower 
than the conventional by $11.28 \mathrm{~dB}$. The $H$-plane mutual coupling results are shown in figure 70 (c) as $S_{31}$. Again, it is reduced by about $6 \mathrm{~dB}$ compared to the conventional when using DGS shape. $S_{41}$ which represents, orthogonal coupling, is reduced by $8 \mathrm{~dB}$ than the conventional case as shown in figure 70 (d). Table 9, summarized all the obtained results.

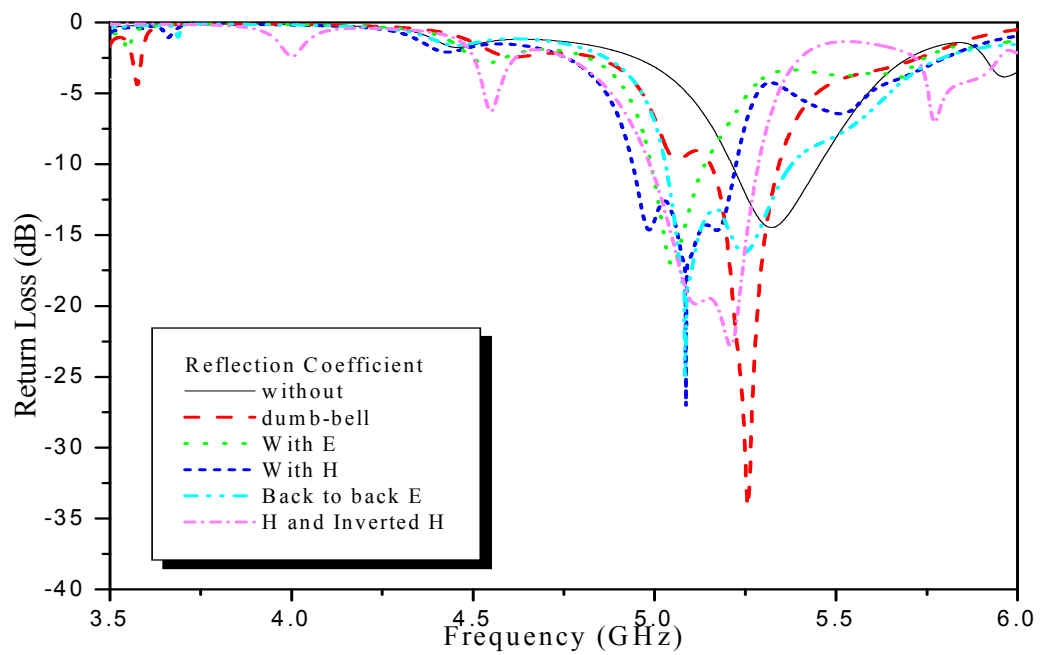

Fig. 66. The reflection coefficient comparison between antenna without and with DGS shapes for line feed array.

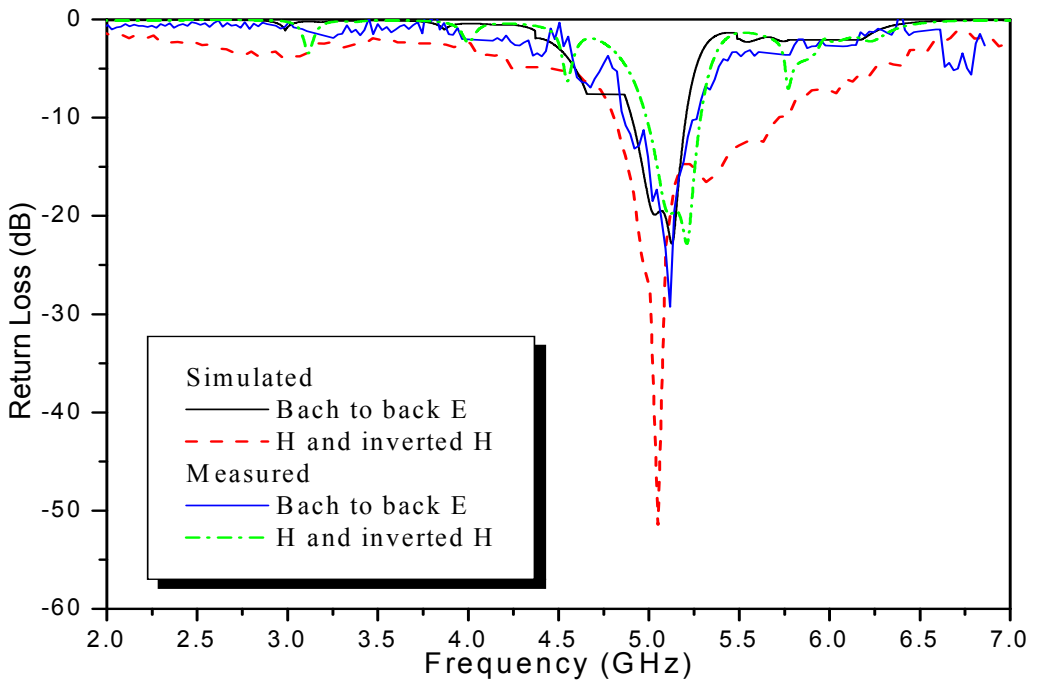

Fig. 67. Comparison between simulated and measured reflection coefficient of MPAA with defect back-to-back $\mathrm{E}$ and $\mathrm{H}$ with inverted $\mathrm{H}$. 


\begin{tabular}{|l|l|l|l|l|}
\hline Antenna Shape & $\begin{array}{l}\text { Resonant Frequency } \\
(\mathrm{GHz})\end{array}$ & $\mathrm{S}_{11}(\mathrm{~dB})$ & BW(MHz) & $\begin{array}{l}\text { Antenna Gain } \\
(\mathrm{dBi})\end{array}$ \\
\hline Without Defect & 5.3 & -15 & 200 & 8.7 \\
\hline With dumb-bell & 5.25 & -18 & 250 & 9 \\
\hline With H & 5.15 & -17 & 175 & 9.5 \\
\hline With E & 5.17 & -30 & 270 & 9.2 \\
\hline Back to Back E & 5.15 & -28 & 350 & 10.2 \\
\hline H and Inverted H & 5.23 & -20 & 200 & 9.7 \\
\hline
\end{tabular}

Table 8 . The characteristics of the microstrip line feed array.

\begin{tabular}{|l|l|l|l|l|}
\hline Antenna Shape & $\mathrm{F}_{\mathrm{o}}(\mathrm{GHz})$ & $\begin{array}{l}\text { Frequency response } \\
\mathrm{S}_{11}, \mathrm{~S}_{21}, \mathrm{~S}_{31}, \mathrm{~S}_{41}(\mathrm{~dB})\end{array}$ & $\mathrm{BW}(\mathrm{MHz})$ & $\begin{array}{l}\text { Antenna Gain } \\
(\mathrm{dB})\end{array}$ \\
\hline Without Defect & 4.75 & $-11,-13,-9.63,-12$ & 150 & 4 \\
\hline With Dimple & 4.7 & $-14,-15,-22,-25$ & 200 & 4.2 \\
\hline With H & 4.65 & $-15,-20,-20,-25$ & 225 & 4.2 \\
\hline With E Shape & 4.4 & $-14,-20,-21,-25$ & 200 & 4.3 \\
\hline Back to Back E & 4.63 & $-20,-20,-20,-25$ & 250 & 5 \\
\hline H and Inverted H & 4.25 & $-17,-20,-20,-25$ & 200 & 6.5 \\
\hline
\end{tabular}

Table 9. The characteristics of MPAA with coaxial feed
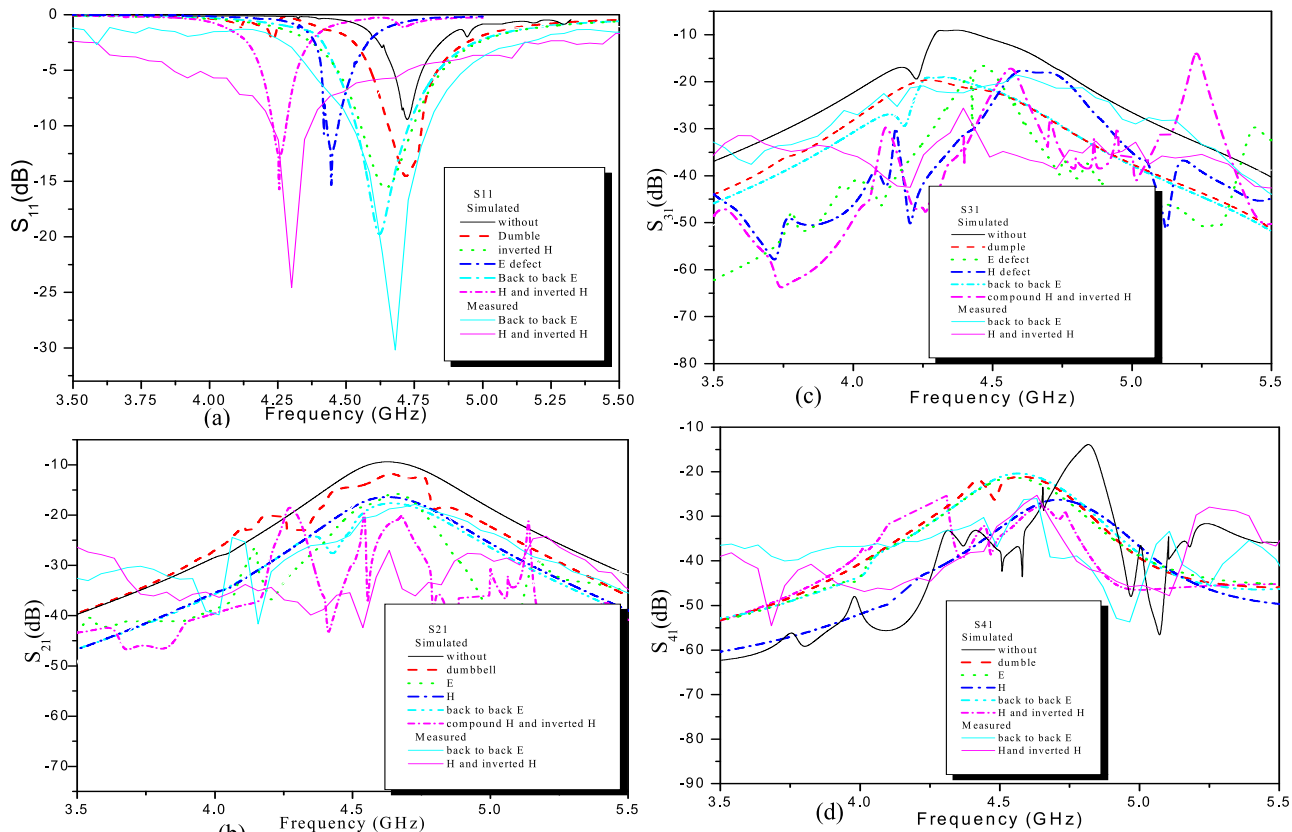

(b)

Fig. 68. The $2 X 2$ coaxial MPAA response, (a) $S_{11}$, (b) $S_{21}$, (c) $S_{31}$ and (d) $S_{41}$. 


\subsubsection{Influence of the different shapes of DGS on the MPAA performance}

From above discussion and results in last sections, it can be concluded that:

- $\quad$ 1- Etching DGS on the ground plane in the middle way between the array patches improves both E-and H-plane coupling. This improvement is due to the surface waves suppression because they do not propagate along the E-Plane direction. So, the mutual coupling of MPAA is determined by both the directions of surface waves and antenna size.

- 2- The new shapes of DGS as back to back $E$ and $H$ with inverted $H$ give better performance than conventional DGS shapes as dumb-bell shape.

The MPAA with back-to-back E and H with inverted H DGS shapes are fabricated using photolithographic techniques as shown in figure 71 . The measured results show good agreement with simulated ones.

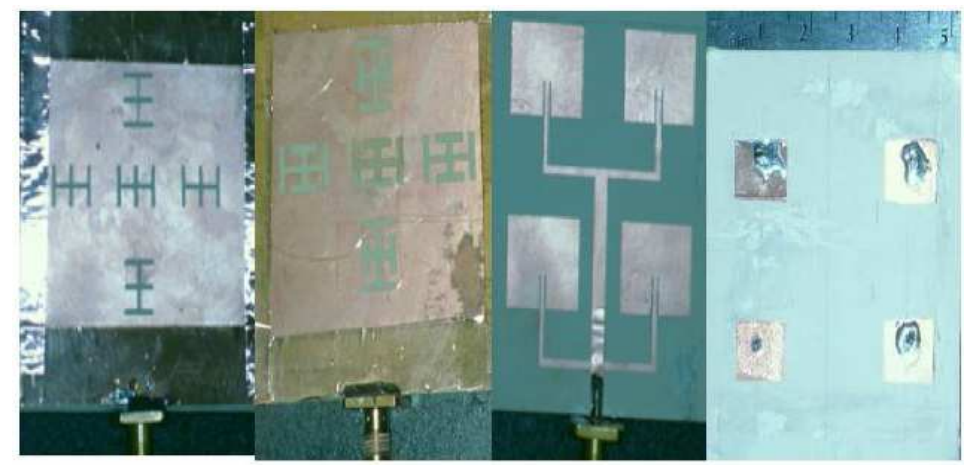

(a)

(b)

(c)

(d)

Fig. 69. (a) The back-to-back E, (b) H and inverted H, (c) inset line feed array and (d) coaxial feed array.

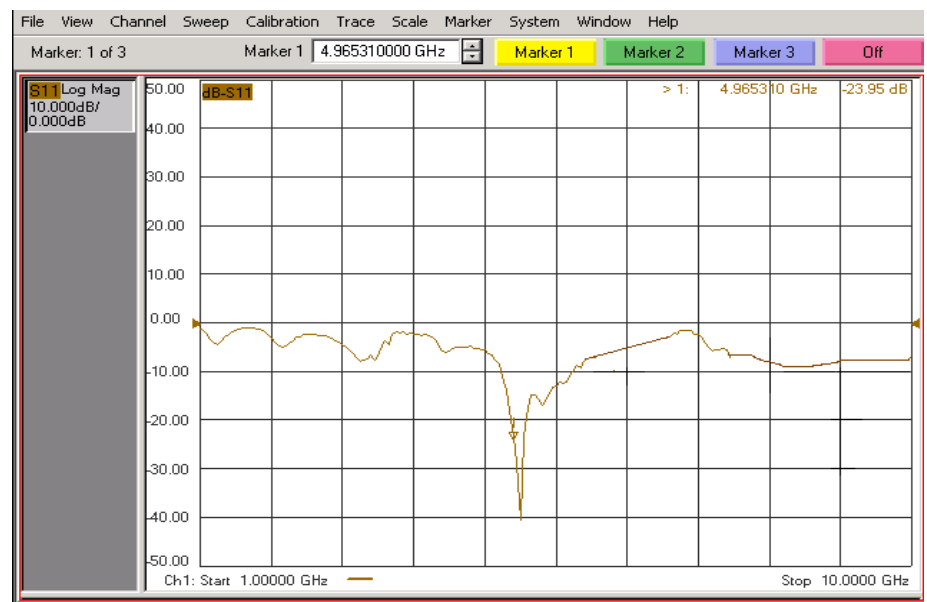

Fig. 70. The measured reflection coefficient of line feed MPAA. 


\subsubsection{Influence of the DGS on other antenna array characteristics}

1. Harmonic Control

Harmonic radiation is a drawback of active integrated MPAA. DGS structures are suggested to reduce the higher-order harmonics in the MPAA. The DGS antenna strongly eliminates the harmonic resonances as shown in figure 72.

\section{PIFA Array Design}

For further reduction in the array size, planar inverted F antenna (PIFA) are used for array's by using patch length less than $\lambda_{0} / 4$. The same dimensions length and width of the coaxial array patch as previous design are kept the same but shorting wall at the top end edge of each element is added. Figure 73 shows the frequency response of the $2 \times 2$ PIFA array with same excitation conditions. From the results, the newly DGS shapes improved the array by more than $5 \mathrm{~dB}$ in S-parameters as compared with the conventional PIFA array. The reduction in array size is around $55 \%$ as compared to conventional array.
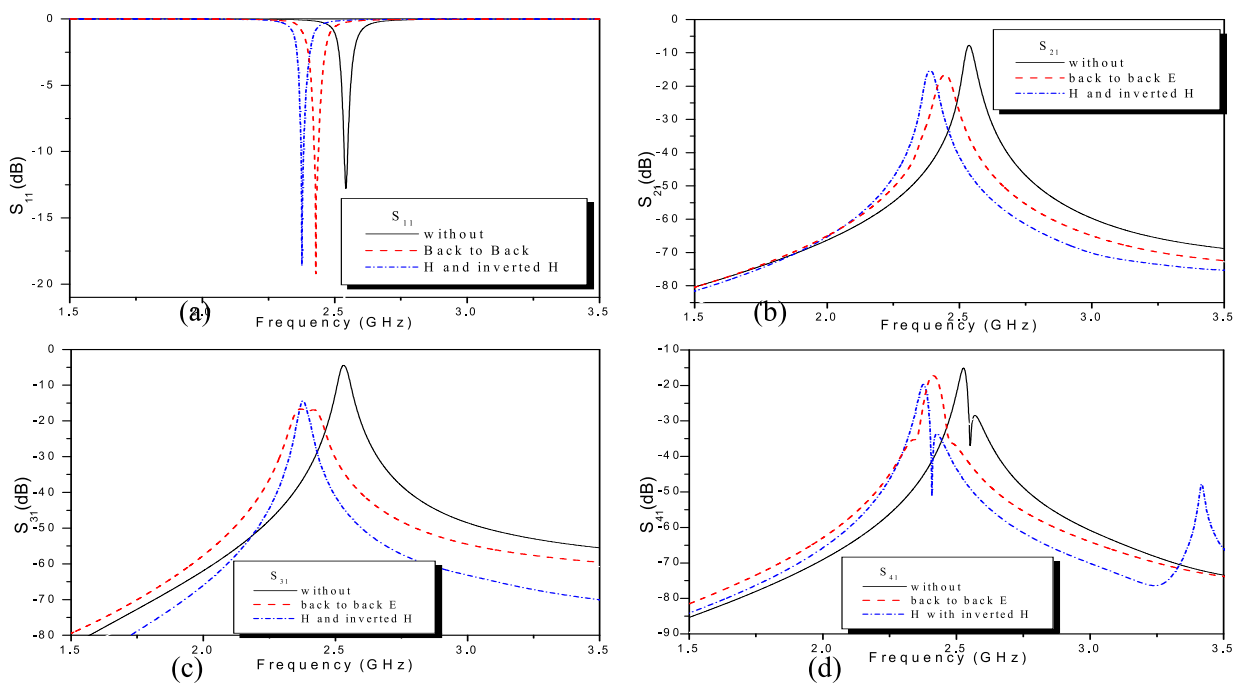

Fig. 71. The 2x2 microstrip PIFA array responses without and with back-to-back $\mathrm{E}$ and $\mathrm{H}$ with I DGS.

\subsection{Ultra-wide bandwidth 2x2 MPAA by using EBG}

Various types of EBG structures have been studied. In one of the first applications, a planar antenna mounted onto an EBG substrate was considered to increase the overall radiation efficiency of the device. Increasing antenna directivity was studied using an EBG structure. A compact spiral EBG structure was studied for microstrip antenna arrays. There are diverse forms of EBG structures, and novel designs such as EBG structures integrated with active device and multilayer EBG structures have been proposed recently [98].

Figure 74 shows four types of the spiral EBG structures used in this study. Comparing to other EBG structures such as dielectric rods and holes, the proposed spiral structure has a 
unique feature of compactness, which is important in wireless communication applications. Specifically, in this study, we investigate the use of four shapes of spiral EBG to help increase the bandwidth of a 2x2 MPAA. We choose the shape of a four-arm spiral AMC, figure 74 (b), for complete cancellation of the antenna cross polarization. This four-arm AMC is then embedded with other EBG structures to further improve the performance of the $2 \times 2$ MPAA. First it is embedded with a large four-arms spiral (LSAMC) as shown in figure $74(\mathrm{c})$, then with small spiral SSAMC patch cells with periodicity $\mathrm{P}$ as shown in figure 74(d), and finally with a mushroom-type EBG with spiral patches as shown in figure 74 (e). Obtained results show that the LSAMC design improves the antenna bandwidth and reduces size, while the SSAMC improves the antenna reflection phase as well as reduces the antenna array size. The embedded spiral electromagnetic band gap structure (ESEBG) in figure 74 (e), was found to improve the antenna array bandwidth and gain. Details of the specific design dimensions and the obtained simulation and experimental results are described in the following sections [98]. The concept of spiral ground plane like spiral antenna as given before in the section before,

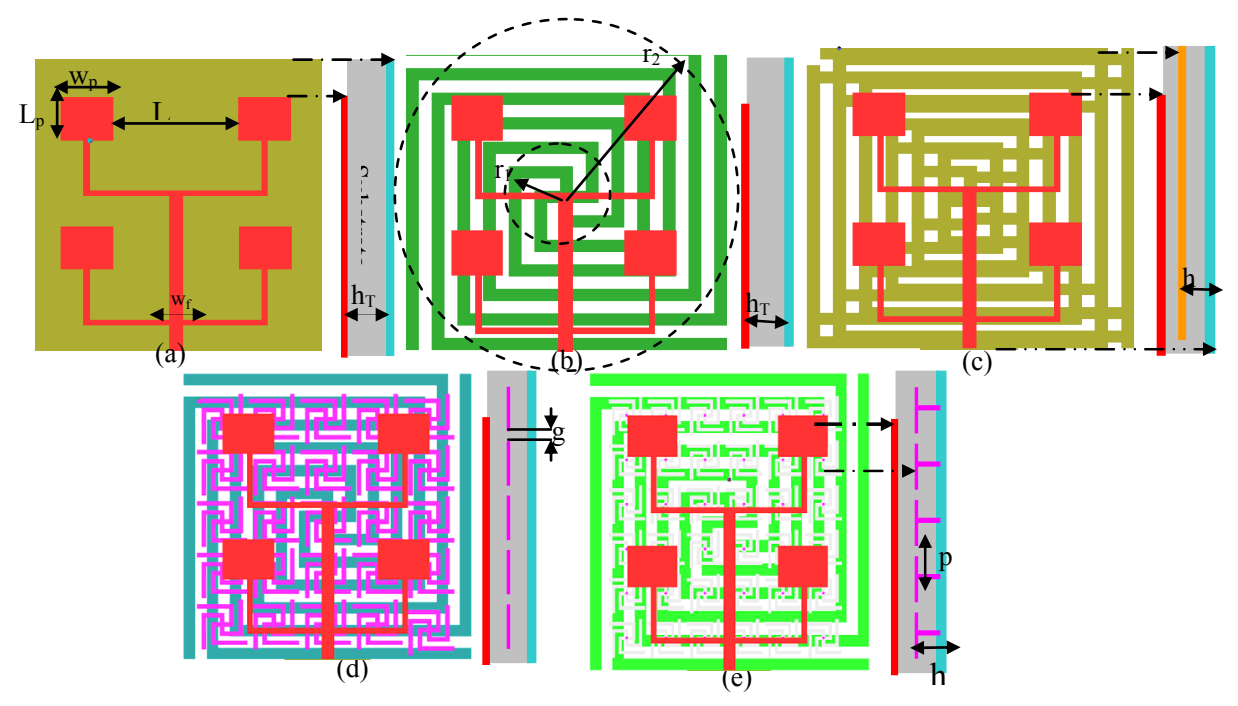

Fig. 72. Different prototype shapes of 2×2 MPAA with different EBG configurations.

\subsubsection{Electromagnetic band-gap structure techniques}

A typical $2 \times 2$ patch antenna array is shown in figure $74(\mathrm{a})$, where single patch length $\mathrm{L}_{\mathrm{p}}$ $=7 \mathrm{~mm}$, width $\mathrm{w}_{\mathrm{p}}=9 \mathrm{~mm}$, with patch separation $\mathrm{L}_{\mathrm{B}}=18 \mathrm{~mm}=0.35 \lambda_{5.2 \mathrm{GHz}}$ and substrate thickness $h_{T}=2.5 \mathrm{~mm}$ with conventional rectangular ground plane of dimension $50 \times 50 \mathrm{~mm}^{2}$. The material of the substrate is RT/D6010 of dielectric constant $\varepsilon_{\mathrm{r}}=10.2$. Figure 74 (b) represents the first type of EBG with four arms spiral AMC ground plane. The dimensions of the ground spiral arms, according to optimization of the transmission coefficient response of the spiral, is arm width equal to separation between arms $=2 \mathrm{~mm}$ and it rotates outward counter clockwise. 
Figure 74 (c) presents prototype array antenna with second type of EBG as embedded large spiral AMC in the middle of the substrate $h=1.25 \mathrm{~mm}$ with previous ground. The dimensions of the embedded spiral is kept the same of the ground plane but less number of spiral turns and centered under the $2 \times 2$ array antenna. To add further improvement in antenna response, small cells of spiral patches with patch size dimensions $6 \times 6 \mathrm{~mm}^{2}$ with spiral arm width equal to gap separation $=0.5 \mathrm{~mm}$ and periodicity $\mathbf{P}=7.5 \mathrm{~mm}$ are added at the same substrate height $\mathbf{h}=1.25 \mathrm{~mm}$ with same pervious ground as shown in figure $74(\mathrm{~d})$. The transmission coefficient response for this spiral structure is also shown in figure 75 . From figure 75, embedded EBG gives best transmission performance followed by embedded small cells spiral with AMC then embedded large spiral AMC and the worst response is for spiral AMC in ground plane. Finally, embedded small four arms spiral patches are used as electromagnetic band-gap structure (EBG) with same pervious dimensions and with vias in the dielectric layer with radius $0.25 \mathrm{~mm}$.

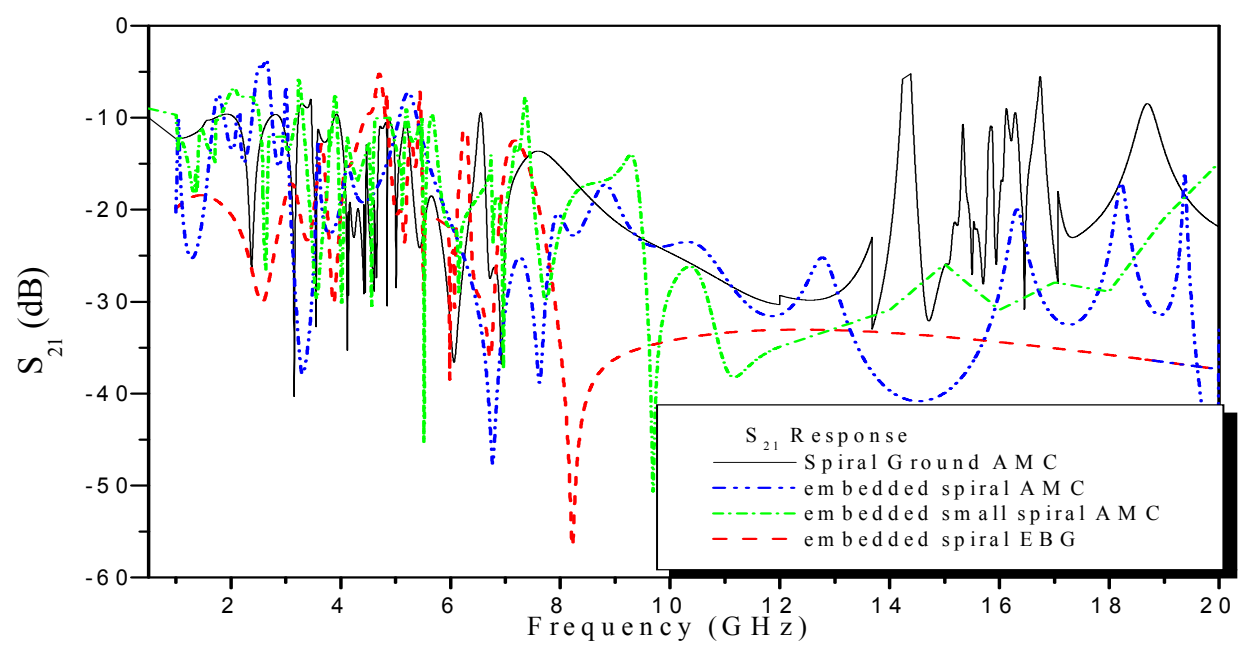

Fig. 73. The transmission response for spiral AMC ground, embedded spiral AMC, embedded small spiral AMC and embedded spiral EBG.

\subsubsection{Antennas results and discussion}

We started with conventional 2x2 MPAA with resonant frequency $5.2 \mathrm{GHz}$. The array resonates at $5.2 \mathrm{GHz}$ with average gain $6.5 \mathrm{dBi}$ and the first harmonic appears at $7.8 \mathrm{GHz}$ then the conventional ground plane is reduced by four arms spiral AMC ground plane. The antenna size is reduced by $50 \%$ and the bandwidth extended from 2.5 to $19 \mathrm{GHz}$ with bandwidth discontinuities in the operating antenna sub-bands as shown in figure 76. Figure 77 shows the comparison between measured and simulated reflection coefficient of array antenna with spiral ground. The antenna bandwidth extended from $3 \mathrm{GHz}$ to $19 \mathrm{GHz}$ with discontinuities in bandwidth, average antenna gain is $7.8 \mathrm{dBi}$. For further improvement in antenna performance as bandwidth and antenna size reduction, another 
embedded spiral with less number of arm turns are added at height $1.25 \mathrm{~mm}$ from the spiral ground plane. The antenna bandwidth extended from 1.25 to $19 \mathrm{GHz}$ with decreased number of sub-band discontinuities as shown in figure 77 with average antenna gain $8.8 \mathrm{dBi}$. Thirdly, small spiral patches are added with periodicity $\mathbf{P}=7.5 \mathrm{~mm}$ to improve the bandwidth discontinuities and reflection phase especially at antenna operating frequency at $5.2 \mathrm{GHz}$. To achieve optimum performance, $2 \mathrm{a}=\lambda_{\mathrm{g}}$ where $\mathbf{a}$ is the side of embedded patch and $\lambda_{\mathrm{g}}$ is the guided wavelength. The bandwidth extended from 0.75 to $20 \mathrm{GHz}$ as shown in figure 77 with average antenna gain increased to $9 \mathrm{dBi}$, so it is increased from original gain by about $4 \mathrm{dBi}$.

Embedded electromagnetic band gap structure with four arm spiral patches are added at the same height to improve the antenna gain to $15.5 \mathrm{dBi}$ and good extended bandwidth without any discontinuities from 0.5 to 3.5 and from 4 to $19 \mathrm{GHz}$ as shown in figure 78 . Figure 79 shows antenna array gain versus frequency. From this figure, all configurations give antenna gain better than the conventional array used over the entire operating band and the embedded spiral EBG gives the best performance followed by small spiral embedded AMC then the large embedded spiral AMC. To Table 10, summarizes all results of antennas array characteristics. The four different configurations were fabricated as shown in figure 80 by using the photolithographic techniques.

\begin{tabular}{|c|c|c|c|c|c|}
\hline $\begin{array}{c}\text { Antenna } \\
\mathrm{Ch} / \mathrm{cs}\end{array}$ & $\begin{array}{l}\text { Convention } \\
\text { al Antenna }\end{array}$ & $\begin{array}{c}\text { Antenna with } \\
\text { SAMC } \\
\text { Ground }\end{array}$ & $\begin{array}{l}\text { Antenna } \\
\text { with E } \\
\text { LSAMC }\end{array}$ & $\begin{array}{c}\text { Antenna with } \\
\text { E } \\
\text { SSAMC }\end{array}$ & $\begin{array}{l}\text { Antenna } \\
\text { ESEBG }\end{array}$ \\
\hline Res. Freq. & $5.25 \mathrm{GHz}$ & $2.5 \mathrm{GHz}$ & $2.25 \mathrm{GHz}$ & $1.25 \mathrm{GHz}$ & $0.75 z$ \\
\hline $\begin{array}{c}-10 \mathrm{~dB} \text { BW } \\
(\mathrm{GHz})\end{array}$ & $\begin{array}{c}\text { Fundamenta } \\
1 \text { resonant } \\
5.1-5.3\end{array}$ & $\begin{array}{c}\text { Extended } \\
\text { from } 1 \text { to } 3, \\
\text { from } 3.25 \text { to } 5, \\
\text { from } 5.2 \text { to } 9 \\
\text { and from } 9.5 \\
\text { to } 19 \mathrm{GHz}\end{array}$ & $\begin{array}{c}\text { Extended } \\
\text { from } 1.25 \text { to } \\
3 \text {, from } 4 \text { to } \\
5 \text {, from } 6 \text { to } \\
12 \text { and from } \\
12.5 \text { to } \\
16 \mathrm{GHz}\end{array}$ & $\begin{array}{l}\text { Extended from } \\
1.25 \text { to } 2.5 \text {, from } \\
2.5 \text { to } 3.5 \text { from } 4 \\
\text { to } 5 \text { from } 5 \text { to } 7 \\
\text { and from } 10 \\
\text { to } 17 \mathrm{GHz}\end{array}$ & $\begin{array}{l}\text { Extended } \\
\text { from } 0.5 \text { to } \\
3.5 \text {, and } \\
\text { from } 4 \text { to } \\
19.5 \mathrm{GHz}\end{array}$ \\
\hline $\begin{array}{c}\text { Average } \\
\text { Rad. Effic. }\end{array}$ & 0.96 & 0.75 & 0.8 & 0.8 & 0.85 \\
\hline $\begin{array}{c}\text { Average } \\
\text { Antenna } \\
\text { gain }\end{array}$ & $6.5 \mathrm{dBi}$ & $7.8 \mathrm{dBi}$ & $8.8 \mathrm{dBi}$ & $9 \mathrm{dBi}$ & $10.5 \mathrm{dBi}$ \\
\hline Fabrication & Easy & Easy & Moderate & Moderate & Hard \\
\hline $\begin{array}{l}\text { Reflection } \\
\text { Phase }\end{array}$ & Bad & Medium & Good & Good & Very Good \\
\hline
\end{tabular}

Table 10. Characteristics of various antennas. 


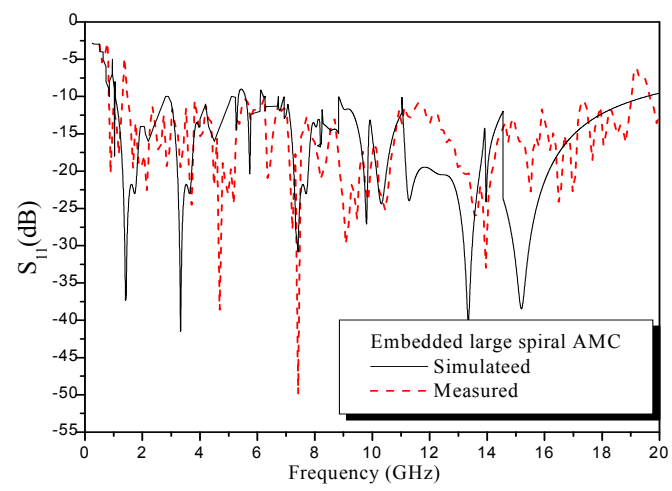

Fig. 74. The comparison between measured and simulated reflection coefficient of embedded large SAMC.

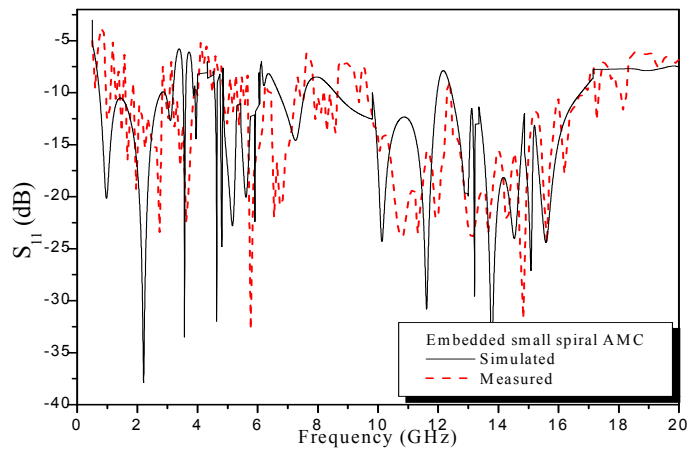

Fig. 75. Comparison between measured and simulated reflection coefficient of embedded small spiral AMC.

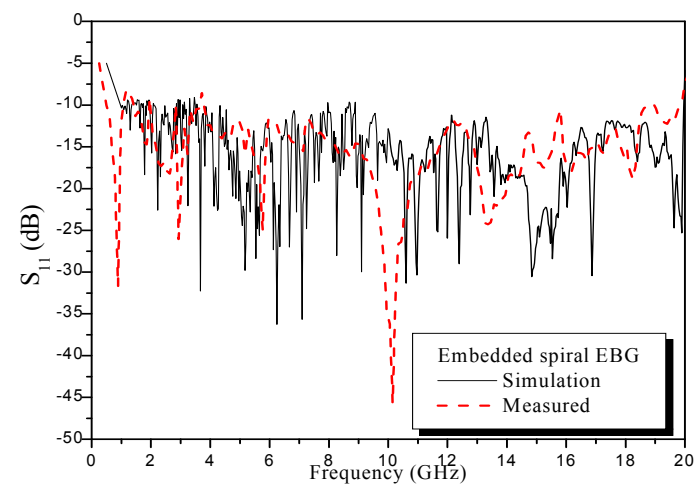

Fig. 76. Comparison between measured and simulated reflection coefficient of embedded small spiral EBG. 


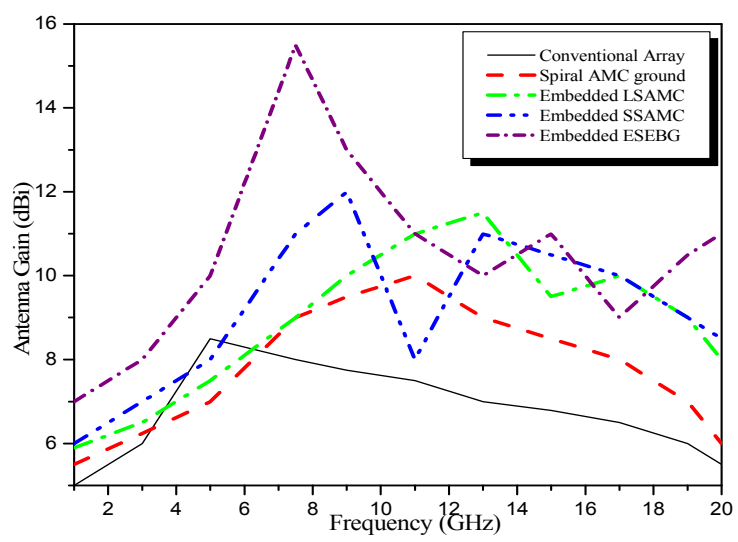

Fig. 77. Array antenna gain -vs-frequency for different configurations.

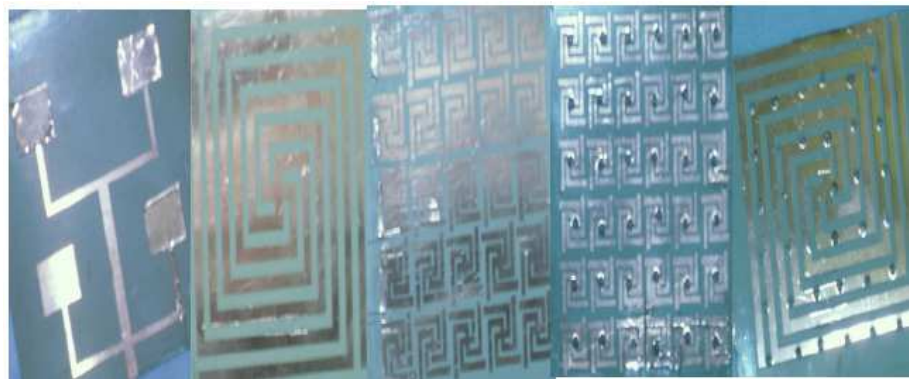

(a)

(b)

(c)

(d)

(e)

Fig. 78. Fabricated 2x2 MPAR(a) Conventional radiator patches, (b) embedded large SAMC, (c) embedded small SAMC, (d) embedded SEBG and (e) ground plane.

\section{References}

[1] C. Caloz, and T. Itoh, Electromagnetic Metamaterials: Transmission Line Theory and Microwave Applications, A John Wiley \& son, Inc., Canada, 2006.

[2] A. Sihvola, "Electromagnetic emergence in metamaterials. Deconstruction of terminology

[3] of complex media", Advances in Electromagnetics of Complex Media and Metamaterials, NATO Science Series II: Mathematics, Physics and Chemistry, Kluwer Academic Publishers, vol. 89, pp. 1 - 17, 2003

[4] A. Sihvola, "Metamaterials in electromagnetics", Metamaterials, vol. 1, no. 1, pp. 2 - 11.

[5] A. Sihvola, "Metamaterials: A Personal View," Radioengineering, vol. 18, no. 2, pp. 90 - 94, 2009.

[6] D. R. Smith, "What are Electromagnetic Metamaterials?," Novel Electromagnetic Materials, Retrieved Auguest, 2010.

[7] http://people.ee.duke.edu/ drsmith/about_metamaterials.html.

[8] J. G. Webster, Metamaterial, Wiley Encyclopedia of Electrical and Electronics Engineering, Jan. 2010. 
[9] V. G. Veselago, “The Eletrodynamics of Substances with Simultaneously Negative Values of $\varepsilon$ and $\mu$," Sov. Phys. Usp, vol. 10, pp. 509 - 514, 1968.

[10] I. V. Lindell, S. A. Tretyakov, K. I. Nikoskinen, and S. Ilvonen, "BW Media-Media with Negative parameters, Capable of Supporting Backward Waves," Microwave Opt. Tech. Lett., vol. 31, no.2, pp. 129-133, 2001.

[11] J. B. Pendry, A. J. Holden,W. J. Stewart, and I. Youngs, "Extremely Low Frequency Plasmons in Metallic Mesostructures," Phys. Rev. Lett., vol. 76, pp. 4773 - 4776, 1996.

[12] J. B. Pendary, A. J. Holden, D. J. Robbins, and W. J. Stewart, "Magnetism from Conductors and Enhanced Nonlinear Phenomena," IEEE Trans. Microwave Theory Tech., vol. 47, pp. 2075 - 2084, 1999.

[13] D. R. Smith, W. Padilla, D. C. Viers, S. C. Nemat-Nasser, and S. Schultz, "A Composite Medium with Simultaneously Negative Permeability and Permittivity," Phys. Rev. Lett., vol. 84, pp. 4184 - 4187, 2000.

[14] D. V. Smith, and J. B. Pendry, "Homogenization of Metamaterials by Field Averaging," J. Opt. Soc, Qm., vol. 23, no. 3, pp. 391 - 403, 2006.

[15] J. B. Pendry, "Negative Refraction Makes a Perfect Lens," Phys. Rev. Lett., vol. 85, pp. 3966 - 3969, 2000.

[16] M. C. K. Wiltshire, J. V. Hajnal, J. B. Pendry, D. J. Edwards, and C. J. Stevens, "Metamaterial Endoscope for Magnetic Field Transfer: Near Field Imaging with Magnetic Wires," Optics Express, vol. 11, no. 7, pp. 709 - 715, 2003.

[17] N. Katsarakis, T. Koschny, M. Kafesaki, E. N. Economou, and C. M. Soukoulis, "Electric Coupling to the Magnetic Resonance of Split Rring Resonators", Applied Phys. lett., vol. 84, no. 15, pp. 2943 - 2945, 2004.

[18] D. Schurig, J. J. Mock, B. J. Justice, S. A. Cummer, J. B. Pendry, A. F. Starr, and D. R. Smith, "Metamaterial Electromagnetic Cloak at Microwave Frequencies," Science, vol. 314, no. 5801, pp. 977 - 980, 2006.

[19] A. Alu, and N. Engheta, "Polarizabilities and Effective Parameters for Collections of Spherical Nanoparticles Formed by Pairs of Concentric Double-Negative, SingleNegative, and/or Double-Positive Metamaterial Layers," Journal of Applied Physics, vol. 97, no. 9, pp. $0943101-12,2005$.

[20] S. F. Mahmoud, “A New Miniaturized Annular Ring Patch Resonator Partially Loaded by A Metamaterial Ring with Negative Permeability and Permittivity," IEEE Antennas and Wireless Propagation Letters, vol. 3, pp. 19 - 22, 2004.

[21] A. Alu, M. G. Silveirinha, A. Salandrino, and N. Engheta, "Epsilon-Near-Zero Metamaterials and Electromagnetic Sources: Tailoring the Radiation Phase Pattern," Physical Review B, vol. 75, no. 15, 2007.

[22] F. Yang and Y. Rahmat-Samii, "Applications of Electromagnetic Band-Gap (EBG) Structures in Microwave Antenna Designs," Proc. of $3^{\text {rd }}$ International Conference on Microwave and Millimeter Wave Technology, pp.528-31, 2002.

[23] R. Marqués and F. Medina, "An Introductory overview on right-handed metamaterials", Proc. the 27th ESA Antenna Workshop on Innovative Periodic Antennas, Spain, pp. 35-41, 2004.

[24] C. R. Simovski, P. Maagt, and I. V. Melchakova, "High impedance surfaces having resonance with respect to polarization and incident angle," IEEE Trans. Antennas Propagat., Vol. 53, no. 3, pp.908-14, 2005. 
[25] G. Gampala, Analysis and design of artificial magnetic conductors for X-band antenna applications, M.Sc. thesis at The University of Mississippi, 2007.

[26] M. A. Jensen, Time-Domain Finite-Difference Methods in Electromagnetics: Application to Personal Communication, Ph.D. dissertation at University of California, Los Angeles, 1994.

[27] F. Yang and Y. Rahmat-Samii, "Bent monopole antennas on EBG ground plane with reconfigurable radiation patterns," 2004 IEEE APS Int. Symp. Dig., Vol. 2, pp. 18191822, Monterey, CA, June 20-26, 2004.

[28] E. Yablonovitch, "Inhibited Spontaneous Emission in Solid-State Physics and Electronics," Physical Review Letters, vol. 58, no. 20, pp. 2059-2062, 1987.

[29] W. Barnes, T. Priest, S. Kitson, J. Sambles, "Photonic Surfaces for Surface-Plasmon Polaritons", Phys. Rev. B, vol. 54, pp. 6227, 1996.

[30] S. Kitson, W. Barnes, J. Sambles, "Full Photonic Band Gap for Surface Modes in the Visible", Phys. Rev. Lett. , vol. 77, pp. 2670, 1996.

[31] L. Brillouin, Wave Propagation in Periodic Structures; Electric Filters and Crystal Lattices, 2nd ed., Dover Publications, New York (1953)

[32] F. Yang, and Y. Rahmat-Samii, "Reflection Phase Characterizations of the EBG Ground Plane for Low Profile Wire Antenna Applications," IEEE Trans. on Antennas Propag., vol. 51, no. 10, Oct. 2003.

[33] J. R. Sohn, H. S. Tae, J. G. Lee, and J. H. Lee, “Comparative Analysis of Four Types of High Impedance Surfaces for Low Profile Antenna Applications," Ant. and Propagat. Society International Symposium, vol. 1A, pp. 758 - 761, 2005.

[34] B. Wu, B. Li, T. Su, and C.-H. Liang, "Equivalent-circuit analysis and lowpass filter design of split-ring resonator DGS," Journal of Electromagnetic Waves and Applications, Vol. 20, No. 14, 1943-1953, 2006.

[35] J. Chen, Z.-B.Weng, Y.-C. Jiao, and F.-S. Zhang, “Lowpass filter design of Hilbert curve ring defected ground structure,", PIER 70, pp. 269-280, 2007.

[36] R. Sharma, T. Chakravarty, and S. Bhooshan, "Design of a Novel $3 \mathrm{~dB}$ Microstrip Backward Wave Coupler Using Defected Ground Structure," Progress In Electromagnetic Research, PIER 65, pp.261-273, 2006.

[37] V. Radisic, Y. Qian, R. Coccioli, and T. Itoh, "Novel 2-D photonic bandgap structure for microstrip lines," IEEE Microw. Guided Wave Lett., Vol. 8, No. 2, 69-71, Feb. 1998.

[38] F. Falcone, T. Lopetegi, and M. Sorolla, "1-D and 2-D photonic bandgap microstrip structures," Microw. Opt. Technol. Lett., Vol. 22, No. 6, 411-412, Sep. 1999.

[39] S. Zouhdi, Ari Sihvola and A. P. Vinogradov, "Applications of EBG in low profile antenna designs: what have we learned" Springer Netherlands, 2009.

[40] D. Cabric, M.S.W. Chen, D.A. Sobel, J. Yang, R.W. Brodersen, "Future wireless systems: UWB, 60GHz, and integrated Circuits Conference," pp. 793-796 cognitive radios," IEEE Proceedings of the Custom Sept. 2005.

[41] D. Elsheakh, "Electromagnetic Band-Gap (EBG) Structure for Microstrip Antenna Systems (Analysis and Design)", PhD thesis, Ain Shams University 2010.

[42] F. R. Yang, Novel Periodic Structures for Applications to Microwave Circuits, Ph.D. Dissertation, Electrical Engineering Dept., University of California, Los Angeles, 1999. 
[43] M. Bradley “One-dimensional photonic band-gap structures and the analogy between optical and quantum mechanical tunneling," Eur. J. Phys, pp.108-112, 1997.

[44] N. Engheta and R. Ziolkowski, "Metamaterials: Physics and Engineering Explorations", John Wiley \& Sons Inc., 2006.

[45] L. Yang, M. Fan, F. Chen, J. She and Z. Feng, "A novel compact electromagneticbandgap (EBG) structure and its application for microwave circuits", IEEE Trans. Microwave Theory Tech., Vol. 53, No. 1, Jan. 2005.

[46] J. Rarity, and C. Weisbuch, "In micro cavities and photonic bandgaps," Physics and Applications, Kluwer Academic Publishers, Dordrecht, 1996.

[47] Park, J. S., Kim, C. S., Kang, H. T. et al., "A novel resonant microstrip RF phase shifter using defected ground structure", 30th European Microwave Conf., pp.72-75, France, 2000.

[48] K. B. Chung, Hong, S. W. "Wavelength demultiplexers based on the superprism phenomena in photonic crystals" IEEE Trans. Antennas and Propag., vol. 81, pp. 1549-1551, Augst 2002.

[49] C. C. Chang,Y. Qian, and T. Itoh, "Analysis and applications of uniplanar compact photonic bandgap structures," PIER, vol. 41, pp. 211-235, 2003.

[50] Dahrele, S. John and Lee, "Strong Localization of Photons in Certain Disordered Dielectric Super Lattices", Physical Review Letters, vol. 58, pp. 2486 - 2489, June 1985.

[51] H. Kosaka, T. Kawashima, A. Tomita, M. Notomi, T. Tamamura, T. Sato, and S. Kawakami, "Photonic Crystals for Micro Light Wave Circuits Using WavelengthDependent Angular Beam Steering," Appl. Phys. Lett., vol. 74, pp. 1370-1372, Sept. 1999.

[52] D. Sievenpiper, "Chapter 11: Review of Theory, Fabrication, and Applications of High Impedance Ground Planes," in Metamaterials: Physics and Engineering Explorations, edited by N. Engheta and R. Ziolkowski, John Wiley \& Sons Inc., 2006.

[53] G.-H. Li,X.-H. Jiang, and X.-M. Zhong, “A novel defected ground structure and its application to a low pass filter," Microwave and Optical Technology Letters, vol. 48, pp. 453-456, Sep. 2006.

[54] C. S. Kim, J. S. Lim, S. Nam, K. Y. Kang, and D. Ahn, "Equivalent circuit modeling of spiral defected ground structure for microstrip line," Electron. Lett., vol. 38, pp. 1109-1120, 2002.

[55] M. Martinez-Vazquez and R. Baggen, "Characterization of printed EBG surfaces for GPS applications," IEEE Int. Workshop on Antenna Technology Small Antennas and Novel Metamaterials, pp. 5-8, March 2006.

[56] I. Garcia, "Electromagnetic band-gap (EBG) structure in antenna design for mobile communication," Project National Science Foundation, 2002-2003.

[57] Y. Ning, C. Zhining, W. Yunyi and C. M. Y. W, " A novel two-layer compact electromagnetic bandgap (EBG) structure and its applications in microwave circuits", Vol. 46 No. 4 Science in China (Series E) August 2003.

[58] X. Q. Chen, X. W. Shi, Y. C. Guo, and C. M. Xiao, " A novel dual band transmitter using microstrip defected ground structure", Progress In Electromagnetics Research, PIER 83, pp. 1-11, 2008. 
[59] C. S. Kim, J. S. Park, D. Ahn, and J. B. Lim, “A novel 1-D periodic defected ground structure for planar circuits," IEEE Microwave Guided Wave Lett., vol. 10, pp. 131133, Apr. 2000.

[60] M. Fallah-Rad and L. Shafai, "Enhanced performance of a microstrip patch antenna using high impedance EBG structure," IEEE APS Int. Symp. Dig., vol. 3, pp. 982-5, June 2003.

[61] C. C. Chiau, X. Chen, and C. G. Parini, "A microstrip patch antenna on the embedded multi-period EBG structure," Proceeding of the 6th Int. Symp. Antennas, Propagation and EM Theory, pp. 96-106, 2003.

[62] E. Yablonovitch, "Photonic band-gap structures", Journal of the Optical Society of America B, vol. 10, No. 2, pp. 283-295, Feb. 1993.

[63] H. Mosallaei and Y. Rahmat-Samii, "Periodic bandgap and effective dielectric materials in electromagnetics: characterization and applications in nanocavities and waveguides," IEEE Trans. Antennas and Propag., vol. 51, pp. 549-63, April 2003.

[64] M. Rahman and M. Stuchly, "Wide-band microstrip patch antenna with planar PBG structure," in Proc. IEEE APS Dig., vol. 2, pp.486-489, 2001,.

[65] G. Guida, A. de Lustrac, and A. Priou, "An introduction to photonic band gap (PBG) materials", PIER, vol. 41, pp.1-20, 2003.

[66] L. Qing-chun, Z. Fang-ming, and H. E Sai-ling, "A New Photonic Band-Gap Cover for a Patch Antenna with a Photonic Band-Gap Substrate", Journal of Zhejiang University Science, vol. 5, No. 3, pp. 269-273, Mar. 2004.

[67] M. A. I. El-Dahshory "Design and analysis of photonic band gap structures", Master Thesis, Cairo University, 2008.

[68] T. Sundström," Analysis of photonic crystal waveguides by the use of FDTD with regularization", Report presented to Royal Institute of Technology, 2004.

[69] A. Aminian, F. Yang, and Y. Rahmat-Samii, "Bandwidth determination for soft and hard ground planes by spectral FDTD: a unified approach in visible and surface wave regions," IEEE Trans. Antennas Propag., vol. 53, pp.18-28, January 2005.

[70] A. S. Barlevy and Y. Rahmat-Samii, "Characterization of electromagnetic band-gaps composed of multiple periodic tripods with interconnecting vias Concept, analysis, and design," IEEE Trans. Antennas Propag., vol. 49, pp. 343-353, Jun. 2001.

[71] C. C. Chiau, X. Chen, and C. G. Parini, "A multi-period EBG structure for microstrip antennas," Proceedings of 2003 ICAP, vol. 2, pp. 727-730, 2003.

[72] G. Goussetis, A. P. Feresidis, and J. C. Vardaxoglou, "FSS printed on grounded dielectric substrates resonance phenomena, AMC and EBG characteristics," IEEE APS Int. Symp. Dig., vol. 1B, pp. 644-647, July 2005.

[73] G. Goussetis, A. P. Feresidis, and J. C. Vardaxoglou, "Tailoring the AMC and EBG characteristics of periodic metallic arrays printed on grounded dielectric substrate," IEEE Trans. Antennas Propagat., vol. 54, pp. 82-9, Jun. 2006.

[74] J. R. Sohn, K. Y. Kim, and H.-S. Tae, "Comparative study on various artificial magnetic conductor for low profile", PIER 61, pp.27-37, 2006

[75] M. G. Bray and D. H. Werner, "A novel design approach for an independently tunable dual-band EBG AMC surface," IEEE APS Int. Symp. Dig., Vol. 1, pp. 289-292, June 2004.

[76] A.R. Butz "Alternative algorithm for Hilbert's space filling curve", IEEE Trans. On Computers, No. 20, pp.424-442, April 1971. 
[77] P. Feresidis, A. Chauraya, G. Goussetis, J. C. Vardaxoglou and P. de Maagt, "Multiband artificial magnetic conductor surfaces", Proc. IEE Seminar on Metamaterials, for Microwave and (Sub) Millimetre Wave Applications, 24 pp. 1-4, Nov. 2003, London, UK.

[78] H. Sagan, “Space-Filling Curves,” Springer-Verlag, New York, 1994.

[79] V K.J. Vinoy, K.A. Jose, V.K. Varadan, and V.V. Varadan, "Hilbert Curve Fractal Antenna: A Small Resonant Antenna for VHF/UHF Applications," Microwave \& Optical Technology Letters, Vol. 29, pp. 215-219, March 2001.

[80] X. Wang Y. Hao Hall, P.S, "Dual-Band Resonances of a Patch Antenna on UC-EBG substrate," Microwave Conference Proceedings, APMC 2005.

[81] D. Nashaat, H. A. Elsadek, E. Abdallah, H. Elhenawy and M. F. Iskander "Electromagnetic Analyses and an Equivalent Circuit Model of Microstrip Patch Antenna with Rectangular Defected Ground Plane" Proceedings of IEEE international symposium on antenna and propagation AP-S, June 2009.

[82] D. Nashaat, H. A. Elsadek, E. Abdallah, H. Elhenawy and M. F. Iskander "Multiband and Miniaturized Inset Feed Microstrip Patch Antenna Using Multiple SpiralShaped Defect Ground Structure (DGS)" Proceedings of IEEE international symposium on antenna and propagation AP-S, June 2009.

[83] D. Nashaat, H. A. Elsadek, E. Abdallah, H. Elhenawy and M. F. Iskander "Miniaturized and Multiband Operations of Inset feed Microstrip Patch Antenna by Using Novel Shape of Defect Ground Structure (DGS) in Wireless Applications" PIERS 2009 in Moscow Progress in Electromagnetics Research Symposium, August, 2009, Moscow, RUSSIA.

[84] D. Nashaat, H. A. Elsadek, E. Abdallah, H. Elhenawy and M. F. Iskander, "UltraWideband and Miniaturization of the Microstrip Monopole Patch antenna (MMPA) with Modified Ground Plane for Wireless Applications," PIERL journal, Vol. 10, pp.171-184, 2009.

[85] D. Nashaat, H. A. Elsadek, E. Abdallah, H. Elhenawy and M. F. Iskander "UltraWideband Microstrip Monopole Antenna by Using Unequal Arms V- Shaped Slot printed on Metamaterial Surface" Proceedings 3rd International Congress on Advanced Electromagnetic Materials in Microwaves and Optics, London, UK, Aug., 2009.

[86] Roy, S., Foerster, J.R., Somayazulu, V.S., and D.G. Leeper. 2004. “Ultrawideband Radio Design: The Promise of High-Speed, Short-Range Wireless Connectivity." Proceedings of the IEEE. Vol. 92. pp. 295-311. Feb 2004.

[87] D. Nashaat, H. A. Elsadek, E. Abdallah, H. Elhenawy and M. F. Iskander, "Enhancement of Ultra-Wide Band Microstrip Monopole Antenna by Using Unequal Arms V-Shaped Slot Printed on Metamaterial Surface", Microwave and Optical Technology letters, Vol. 52, No. 10, pp:2203-2208, October 2010.

[88] D. Nashaat, H. A. Elsadek, E. Abdallah, H. Elhenawy and M. F. Iskander, "UltraWideband Co-planar Boat Microstrip Patch with Modified Ground Plane by Using Electromagnetic Band-Gap Structure (EBG) for Wireless Communication" published in Microwave antenna Optical Technology letters, Vol 52, issue 5, pp.1159-1164, 2010.

[89] A. Yu and X. Zhang, "A low profile monopole antenna using a dumbbell EBG structure," IEEE APS Int. Symp. Dig., Vol. 2, pp. 1155-8, 20-25 June 2004. 
[90] D. Nashaat, H. A. Elsadek, E.t Abdallah, H. Elhenawy and M. F. Iskander, "Enhancement of Microstrip Monopole Antenna Bandwidth by Using EBG Structures published in IEEE Antennas and Wireless Propag., Letters, vol. 8, pp.959-963, 2009.

[91] D. Nashaat, H. A. Elsadek, E. Abdallah, H. Elhenawy and M. F. Iskander, "Ultra-Wide Bandwidth Umbrella Shaped Microstrip Monopole Antenna Using Spiral Artificial Magnetic Conductor (SAMC)" IEEE Antennas and Wireless Propag., Letters, vol.8, pp.1225-1229, 2009.

[92] D. Nashaat, H. A. Elsadek, E. Abdallah, H. Elhenawy and M. F. Iskander, "Investigated New Embedded Shapes of Electromagnetic Bandgap Structures and Via Effect for Improvement Microstrip Patch Antenna Performance " PIERB journal, vol. 12, pp.90-107, 2010.

[93] D. Nashaat, H. A. Elsadek, E. Abdallah, H. Elhenawy and M. F. Iskander, "Reconfigurable Single and Multiband Inset feed Microstrip Patch Antenna for Wireless Communication Devices" PIERC journal, Vol. 12, pp.191-201, 2010.

[94] F. Yang and Y. Rahmat-Samii, Mutual coupling reduction of microstrip antennas using electromagnetic band-gap structure, Proc IEEE AP-S Dig. 2 pp. 478-481, 2001.

[95] W. Yun and Y. J Yoon, “A Wideband aperture-coupled microstrip array antenna using inverted feeding structures," IEEE Trans. Antenna and Propg., Vol. 53, No. 2, PP: 861-862, Feb. 2005.

[96] R. Gonzalo Garcia, P. de Maagt and M. Sorolla, “Enhanced patch-antenna performance by suppressing surface waves using photonic-band-gap substrate," IEEE Trans. Microwave Theory Tech., Vol. 47, No. 11, PP: 213 1-2138, November 1999.

[97] D. Nashaat, H. A. Elsadek, E. Abdallah, H. Elhenawy and M. F. Iskander, "Microstrip Array Antenna with New 2D-Electromagnetic Band Gap Structure Shapes to Reduce Harmonics and Mutual Coupling", published in PIERC journal, Vol. 20, pp.203-213, 2010.

[98] M. Salehi and A. Tavakoli, A novel low coupling microstrip antenna array design using defected ground structure, Int J Electron Commun 60, pp. 718-723, 2006.

[99] D. Nashaat, H. A. Elsadek, E. Abdallah, H. Elhenawy and M. F. Iskander,, "Low Mutual Coupling 2X2 Microstrip Patch Array Antenna by Using Novel Shapes of Defect Ground Structure (DGS)" published in Microwave antenna Optical Technology letters, Vol 52, issue 5, pp.1208-1215, 2010.

[100] D. Nashaat, H. A. Elsadek, E. Abdallah, H. Elhenawy and M. F. Iskander, "Ultra-Wide Bandwidth 2x2 Microstrip Patch Array Antenna by Using Electromagnetic Bandgap Structure (EBG)", IEEE Trans., Antenna and Propagation, vol.5, pp., 2011.

[101] R. Elliot, "On the Theory of Corrugated Plane Surfaces", IRE Trans. Ant. Prop., vol. 2, pp. $71-81,1954$.

[102] W. Rotman, "A Study of Single-Surface Corrugated Guides", Proc. IRE, vol. 39, pp. 952 - 959, 1951.

[103] P.-S. Kildal, "Artificially Soft and Hard Surfaces in Electromagnetics”, IEEE Trans. Ant. Prop., vol. 38, pp. 1537 - 1544, 1990. 


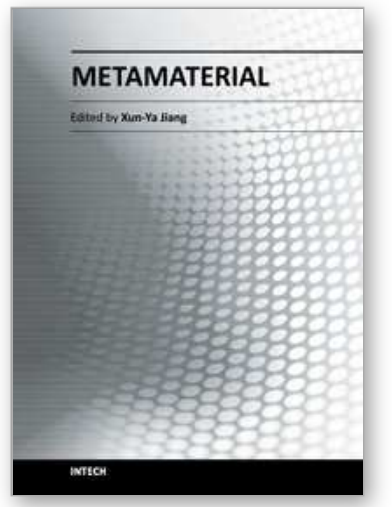

\author{
Metamaterial \\ Edited by Dr. Xun-Ya Jiang
}

ISBN 978-953-51-0591-6

Hard cover, 620 pages

Publisher InTech

Published online 16, May, 2012

Published in print edition May, 2012

In-depth analysis of the theory, properties and description of the most potential technological applications of metamaterials for the realization of novel devices such as subwavelength lenses, invisibility cloaks, dipole and reflector antennas, high frequency telecommunications, new designs of bandpass filters, absorbers and concentrators of EM waves etc. In order to create a new devices it is necessary to know the main electrodynamical characteristics of metamaterial structures on the basis of which the device is supposed to be created. The electromagnetic wave scattering surfaces built with metamaterials are primarily based on the ability of metamaterials to control the surrounded electromagnetic fields by varying their permeability and permittivity characteristics. The book covers some solutions for microwave wavelength scales as well as exploitation of nanoscale EM wavelength such as visible specter using recent advances of nanotechnology, for instance in the field of nanowires, nanopolymers, carbon nanotubes and graphene. Metamaterial is suitable for scholars from extremely large scientific domain and therefore given to engineers, scientists, graduates and other interested professionals from photonics to nanoscience and from material science to antenna engineering as a comprehensive reference on this artificial materials of tomorrow.

\title{
How to reference
}

In order to correctly reference this scholarly work, feel free to copy and paste the following:

Dalia M.N. Elsheakh, Hala A. Elsadek and Esmat A. Abdallah (2012). Antenna Designs with Electromagnetic Band Gap Structures, Metamaterial, Dr. Xun-Ya Jiang (Ed.), ISBN: 978-953-51-0591-6, InTech, Available from: http://www.intechopen.com/books/metamaterial/design-antenna-with-electromagnetic-band-gapstructures

\section{INTECH}

open science | open minds

\section{InTech Europe}

University Campus STeP Ri

Slavka Krautzeka 83/A

51000 Rijeka, Croatia

Phone: +385 (51) 770447

Fax: +385 (51) 686166

www.intechopen.com
InTech China

Unit 405, Office Block, Hotel Equatorial Shanghai

No.65, Yan An Road (West), Shanghai, 200040, China

中国上海市延安西路65号上海国际贵都大饭店办公楼405单元

Phone: +86-21-62489820

Fax: +86-21-62489821 
(C) 2012 The Author(s). Licensee IntechOpen. This is an open access article distributed under the terms of the Creative Commons Attribution 3.0 License, which permits unrestricted use, distribution, and reproduction in any medium, provided the original work is properly cited. 